\title{
Dual photochemical bond cleavage for a diarylethene-based phototrigger containing both
}

methanolic and acetic sources.

Olivier Galangau ${ }^{\dagger}$, Stéphanie Delbaere ${ }^{\ddagger}$, Nicolas Ratel-Ramond ${ }^{\dagger}$, Gwénaël Rapenne ${ }^{\dagger \# *}$, Ruiji Li ${ }^{\S}$, Jan Patrick Dela Cruz Calupitan, ${ }^{\dagger \# \S}$ Takuya Nakashima $^{\S}$ and Tsuyoshi Kawai ${ }^{\dagger \uparrow *}$

${ }^{\dagger}$ International Collaborative Laboratory for Supraphotoactive Systems, NAIST-CEMES, CNRS UPR 8011, 29 rue Marvig, F-31055 Toulouse Cedex 4, France

${ }^{\ddagger}$ Université de Lille; CNRS UMR 8516 LASIR, 3, rue Pr. Laguesse - BP 83 - 59006 Lille Cedex, France

\# Université de Toulouse, UPS, 29 rue Marvig, F-31055 Toulouse Cedex 4, France

$\S$ Graduate School of Materials Science, Nara Institute of Science and Technology, NAIST, 8916-5 Takayama-cho, Ikoma, Nara 630-0192, Japan

\section{Table of content}

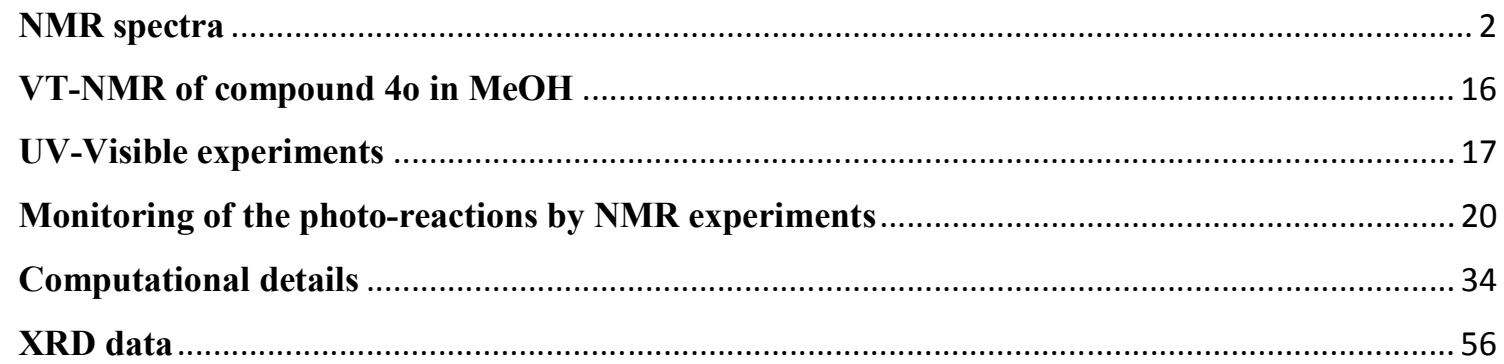




\section{NMR spectra}

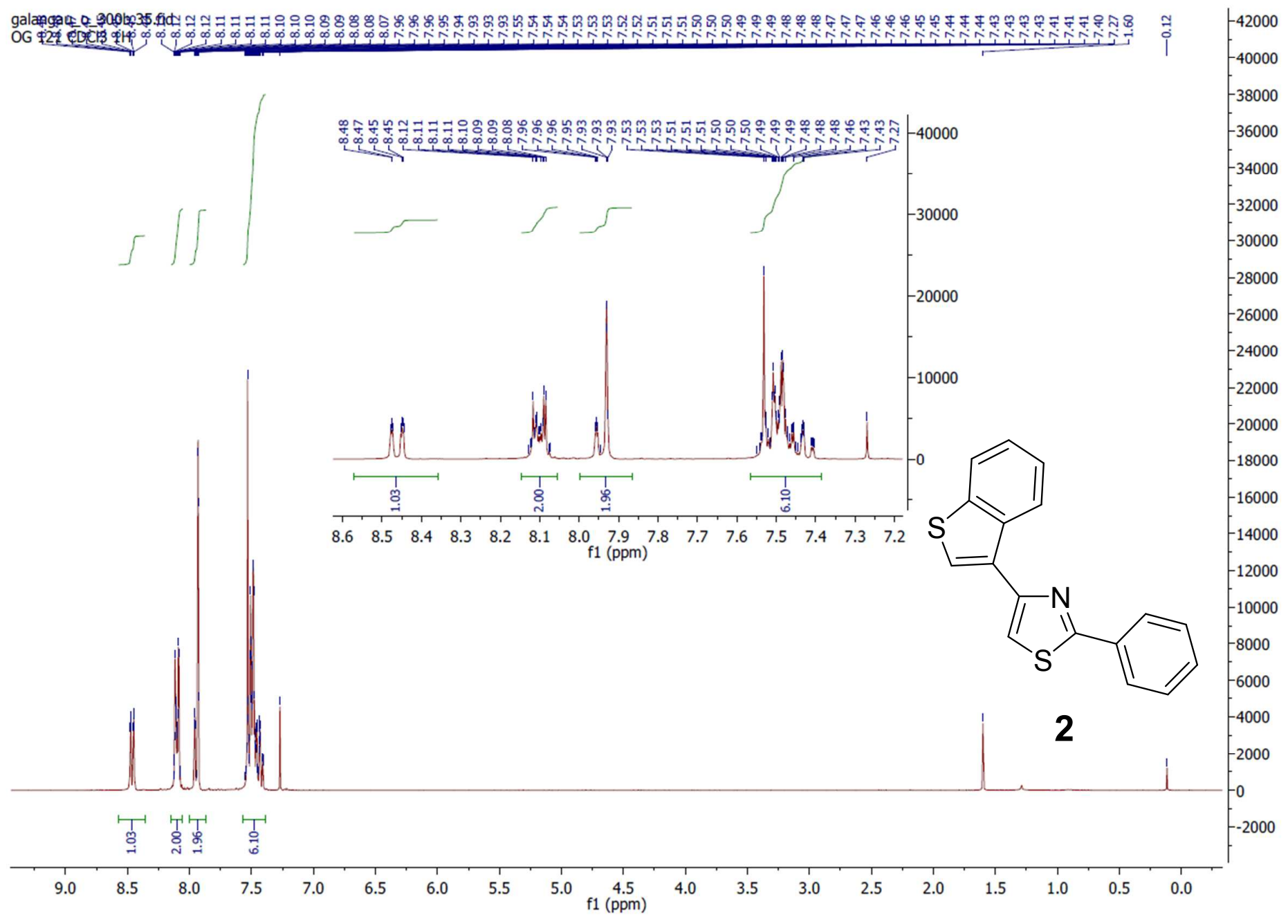

Figure $\mathrm{S}^{1}{ }^{1} \mathrm{H}$-NMR spectrum of compound 2 in $\mathrm{CDCl}_{3}$ 
galangau_o_300b.36.fid
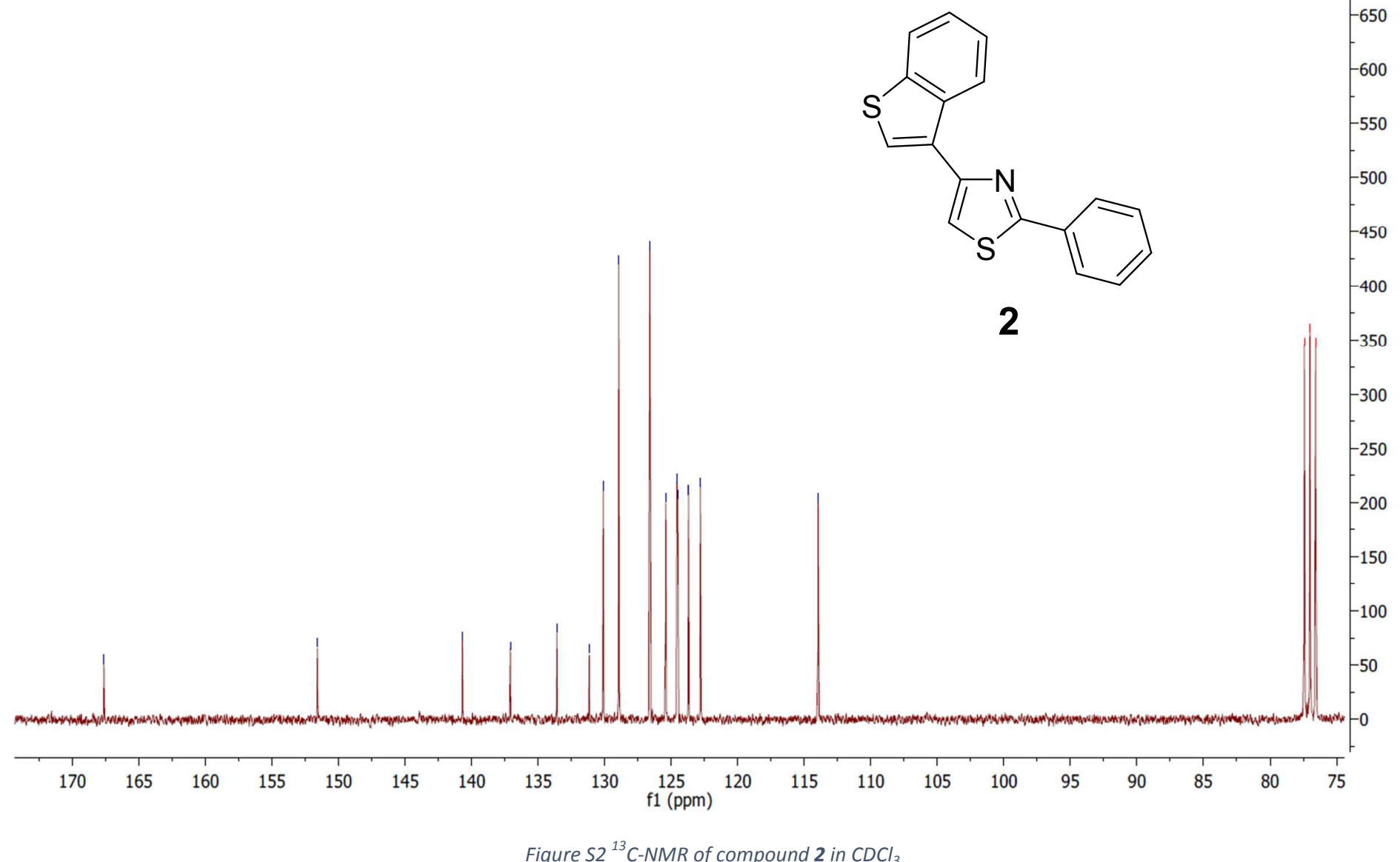


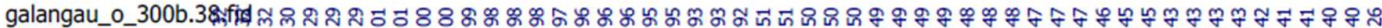

OG1291H 0i

(
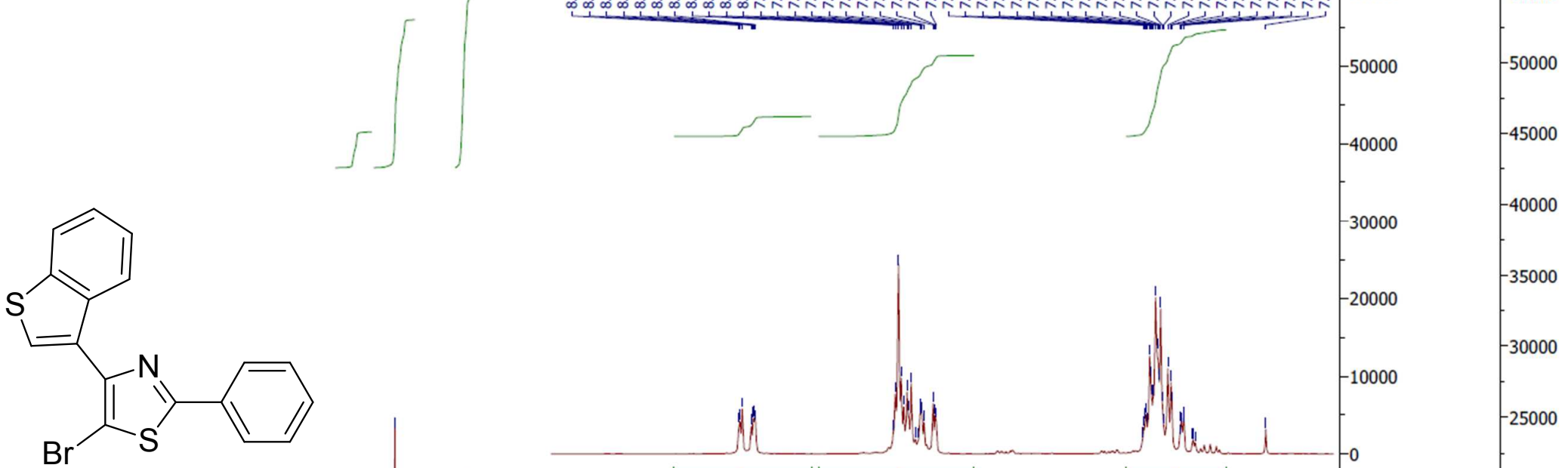

3

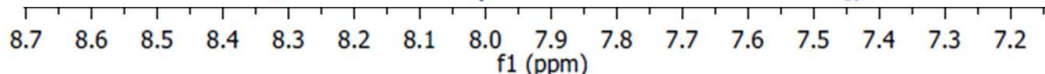

$-10000$

5000

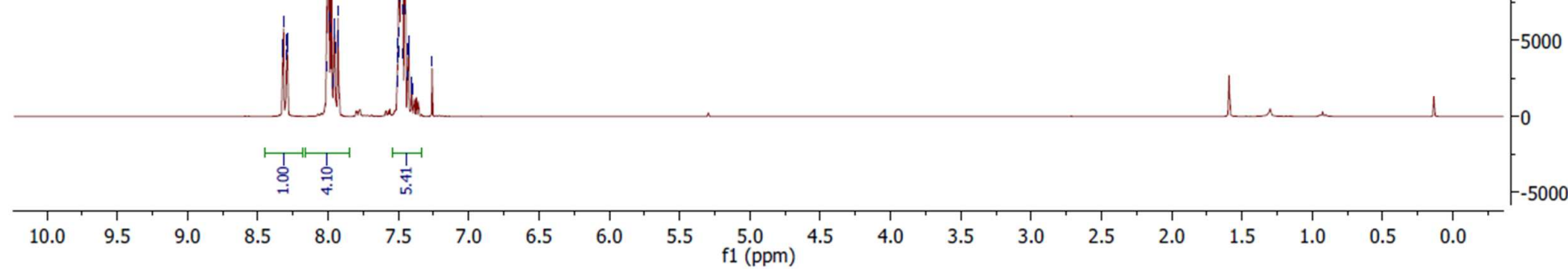

Figure $\mathrm{S} 3{ }^{1} \mathrm{H}-\mathrm{NMR}$ of compound 3 in $\mathrm{CDCl}_{3}$ 


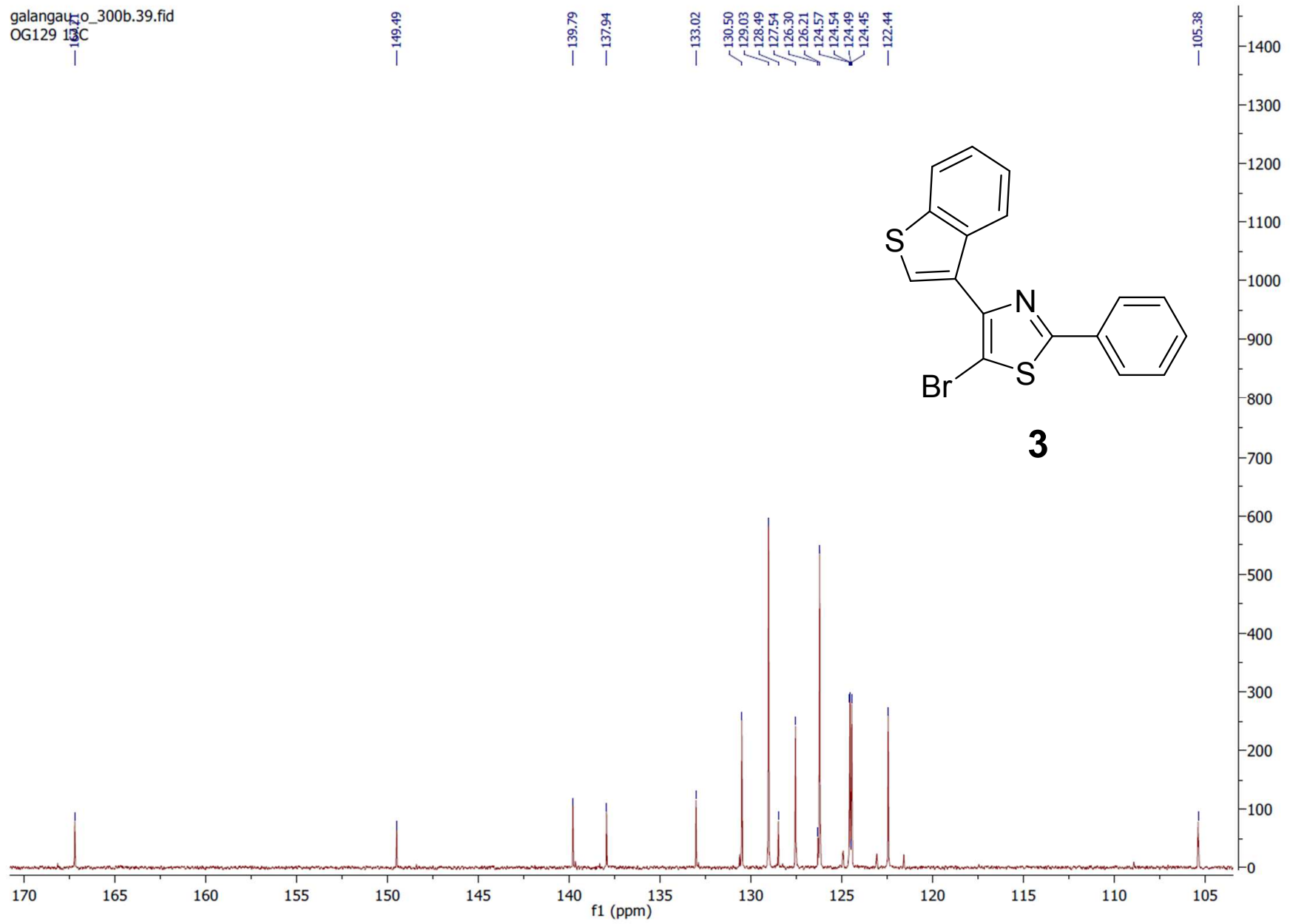

Figure $54{ }^{13} \mathrm{C}-\mathrm{NMR}$ of compound 3 in $\mathrm{CDCl}_{3}$ 


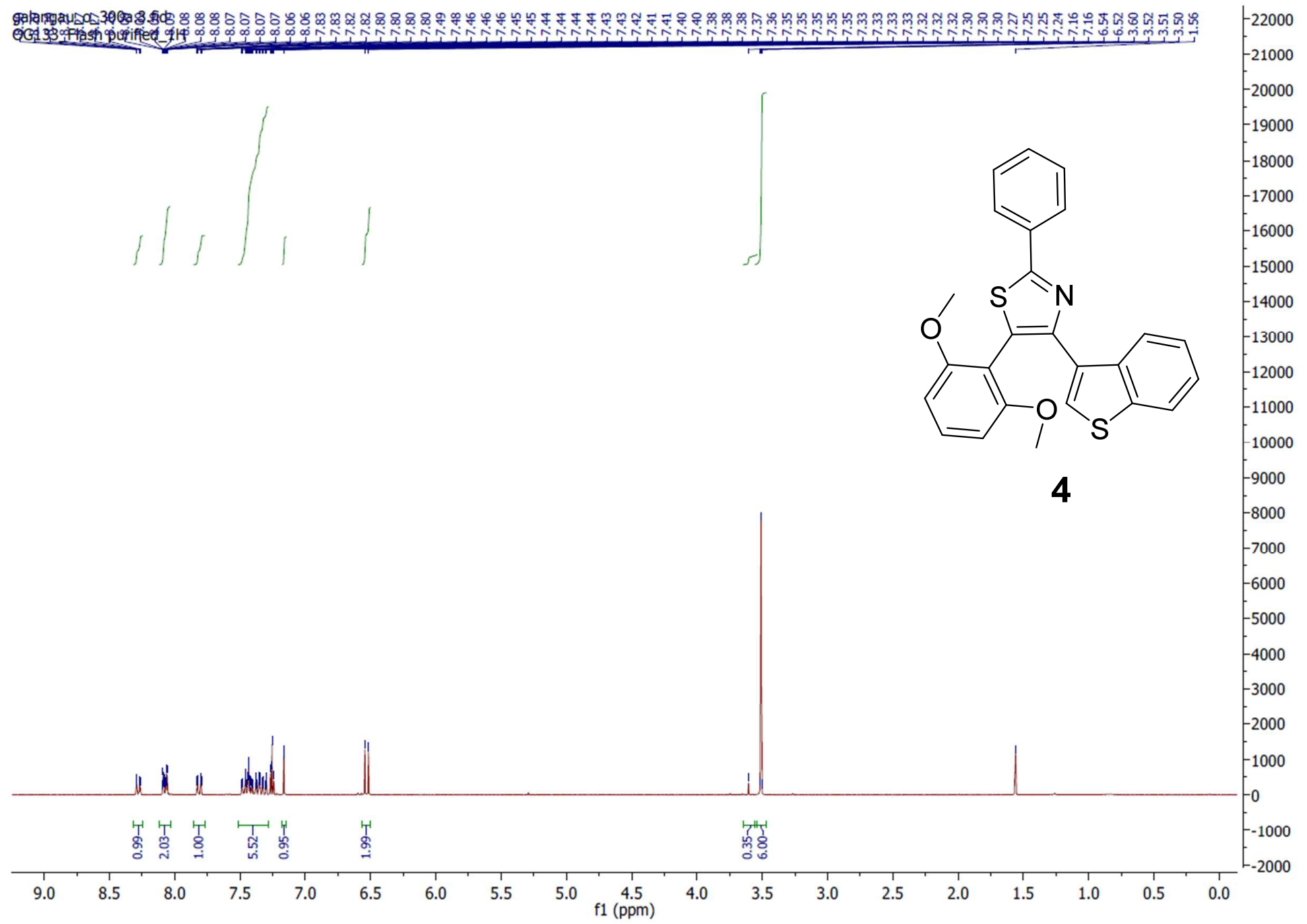

Figure $\mathrm{S}^{1}{ }^{1} \mathrm{H}-\mathrm{NMR}$ of compound 4 in $\mathrm{CDCl}_{3}$ 


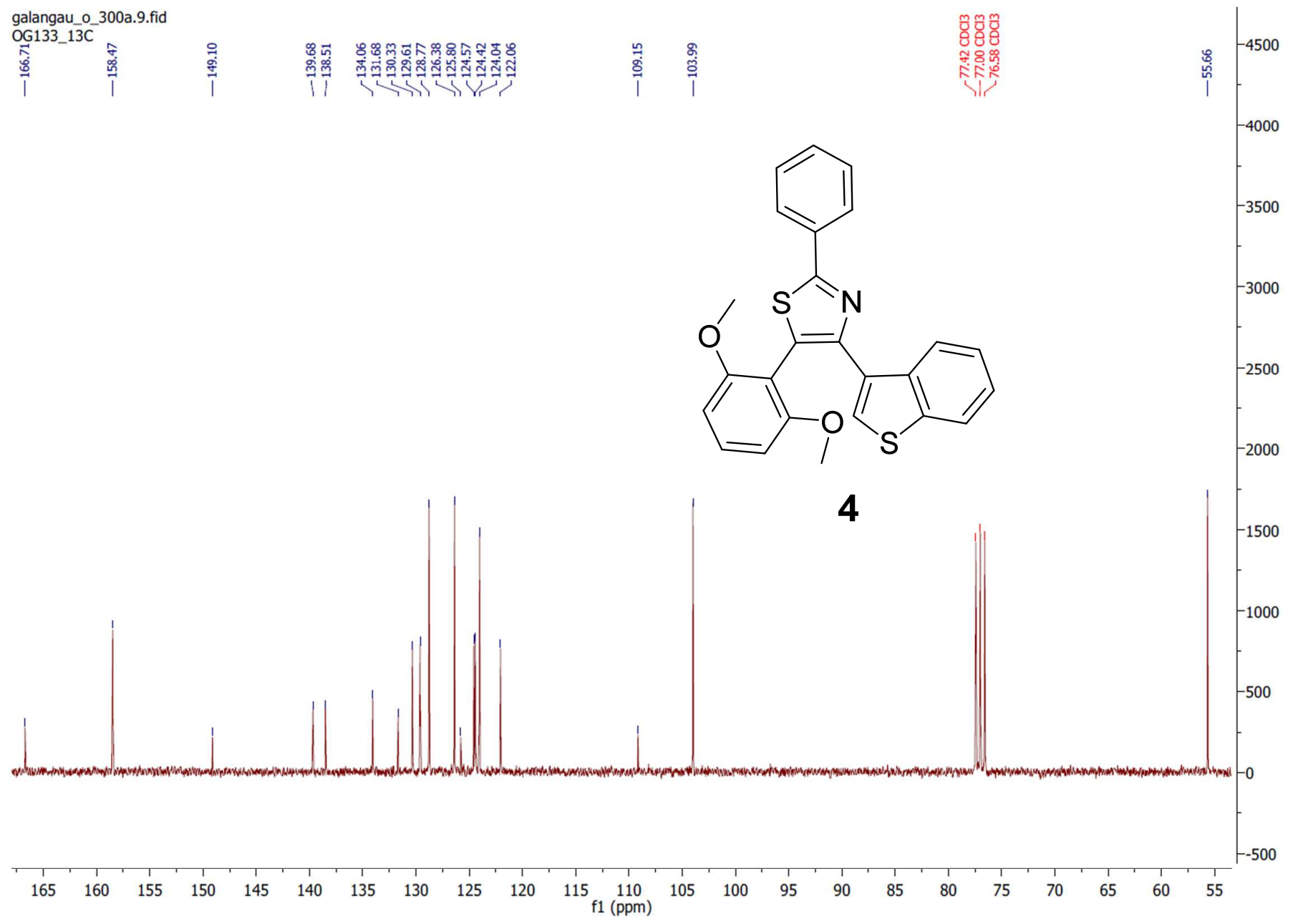

Figure $\mathrm{S}^{13}{ }^{13} \mathrm{C}-\mathrm{NMR}$ of compound 4 in $\mathrm{CDCl}_{3}$ 


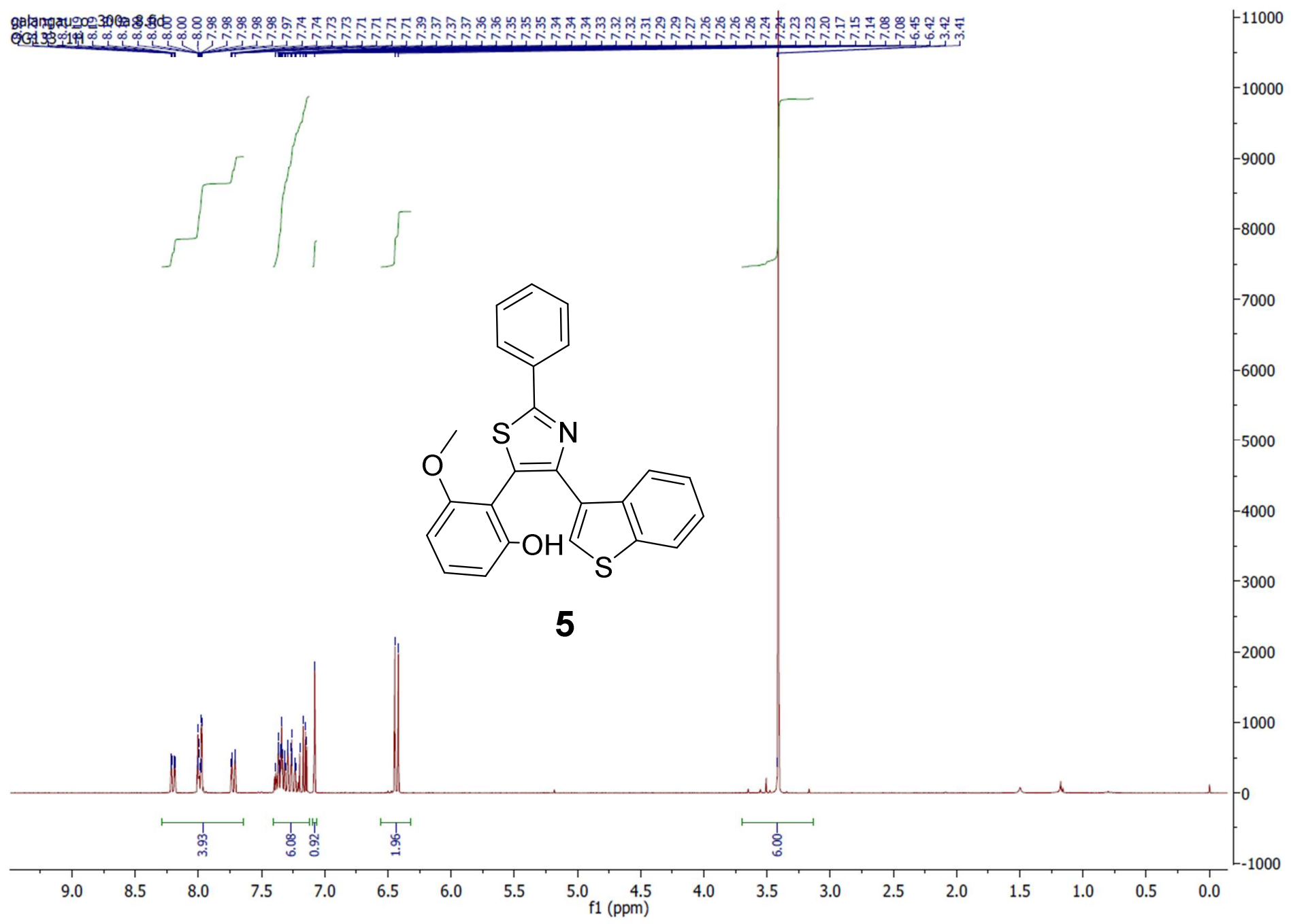

Figure $\mathrm{S}^{1} \mathrm{H}-\mathrm{NMR}$ of compound 5 in $\mathrm{CDCl}_{3}$ 


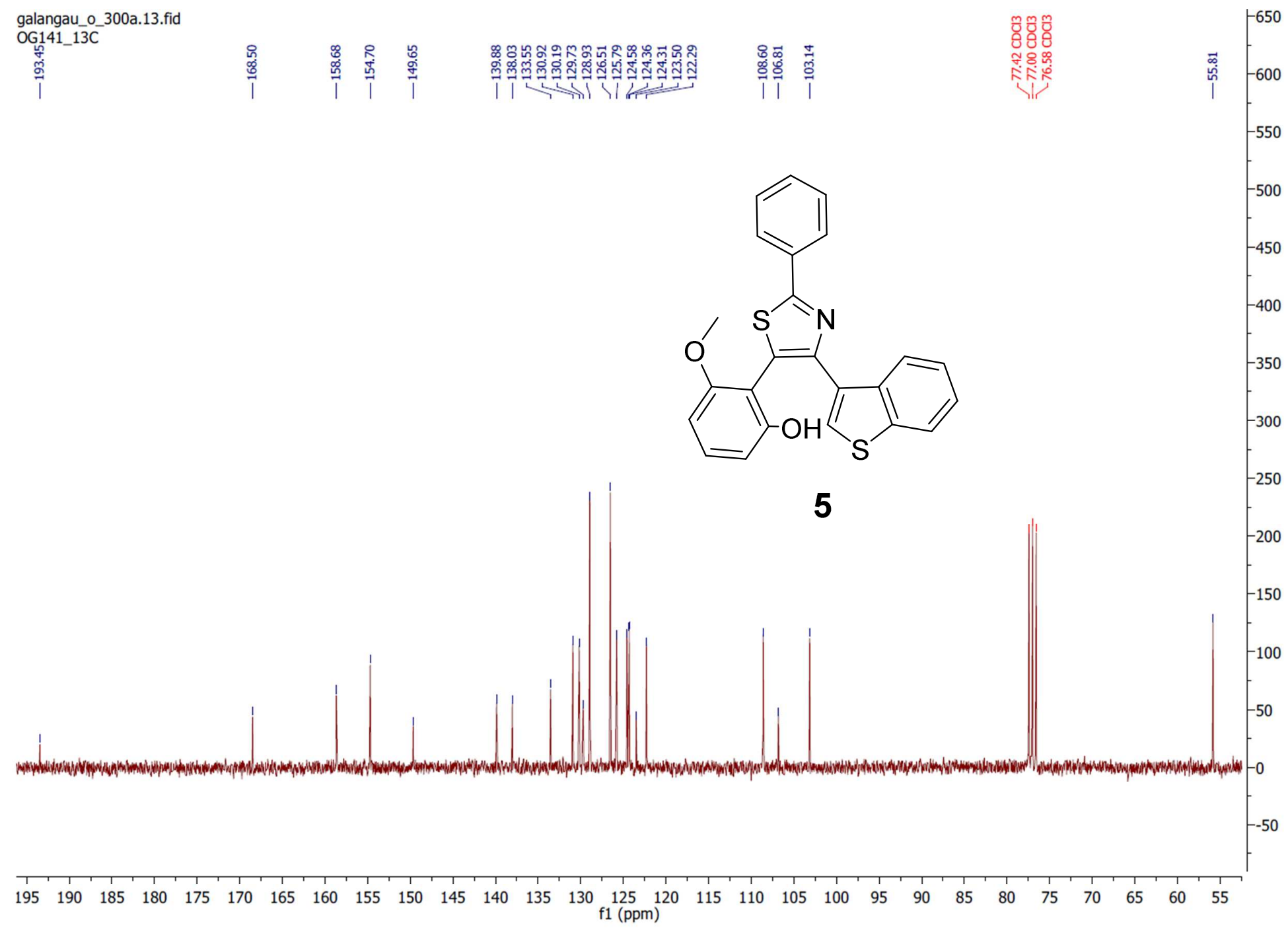

Figure $\mathrm{S}^{13} \mathrm{C}-\mathrm{NMR}$ of compound 5 in $\mathrm{CDCl}_{3}$ 


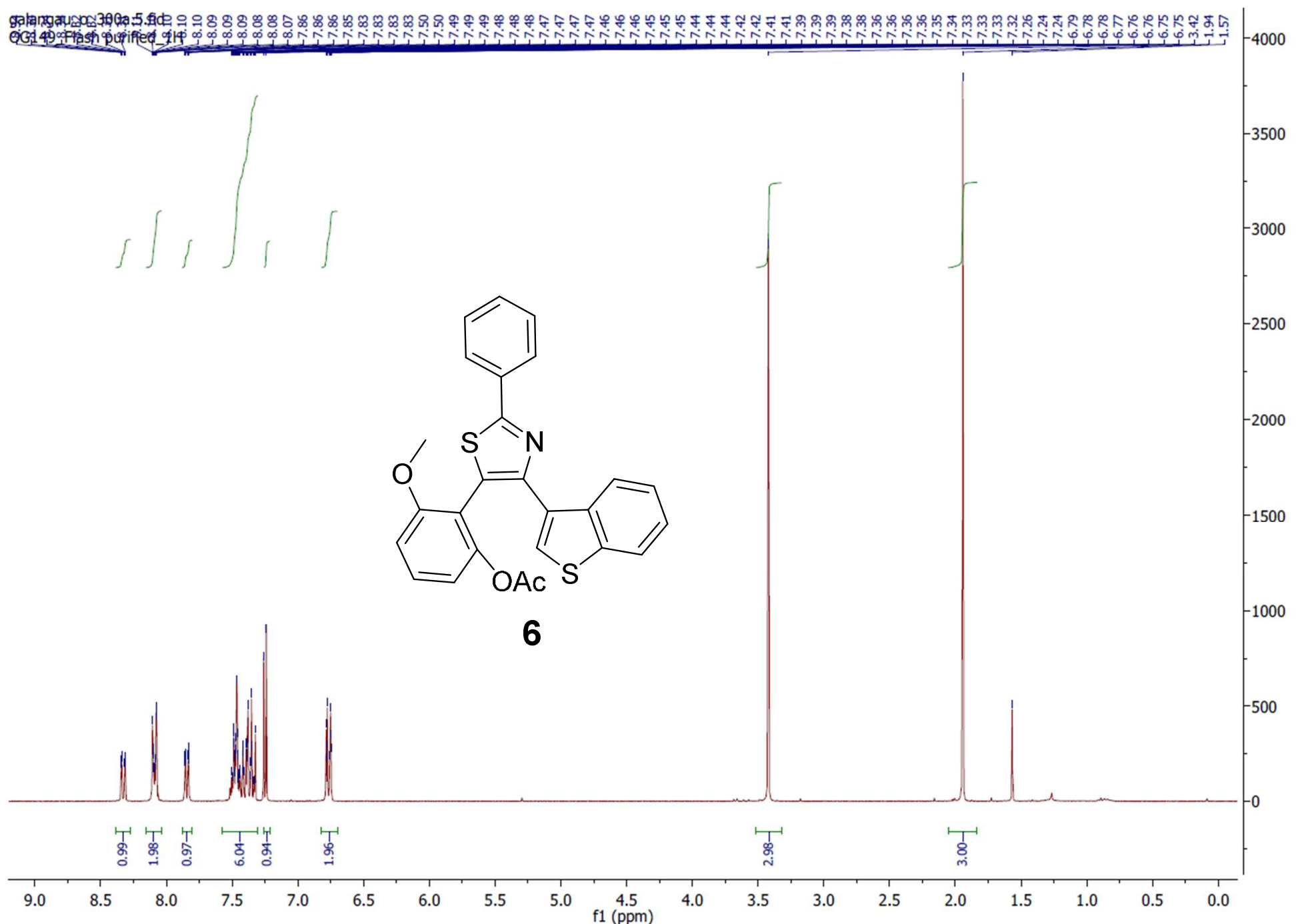

Figure $S 9^{1} \mathrm{H}-\mathrm{NMR}$ of compound 6 in $\mathrm{CDCl}_{3}$ 


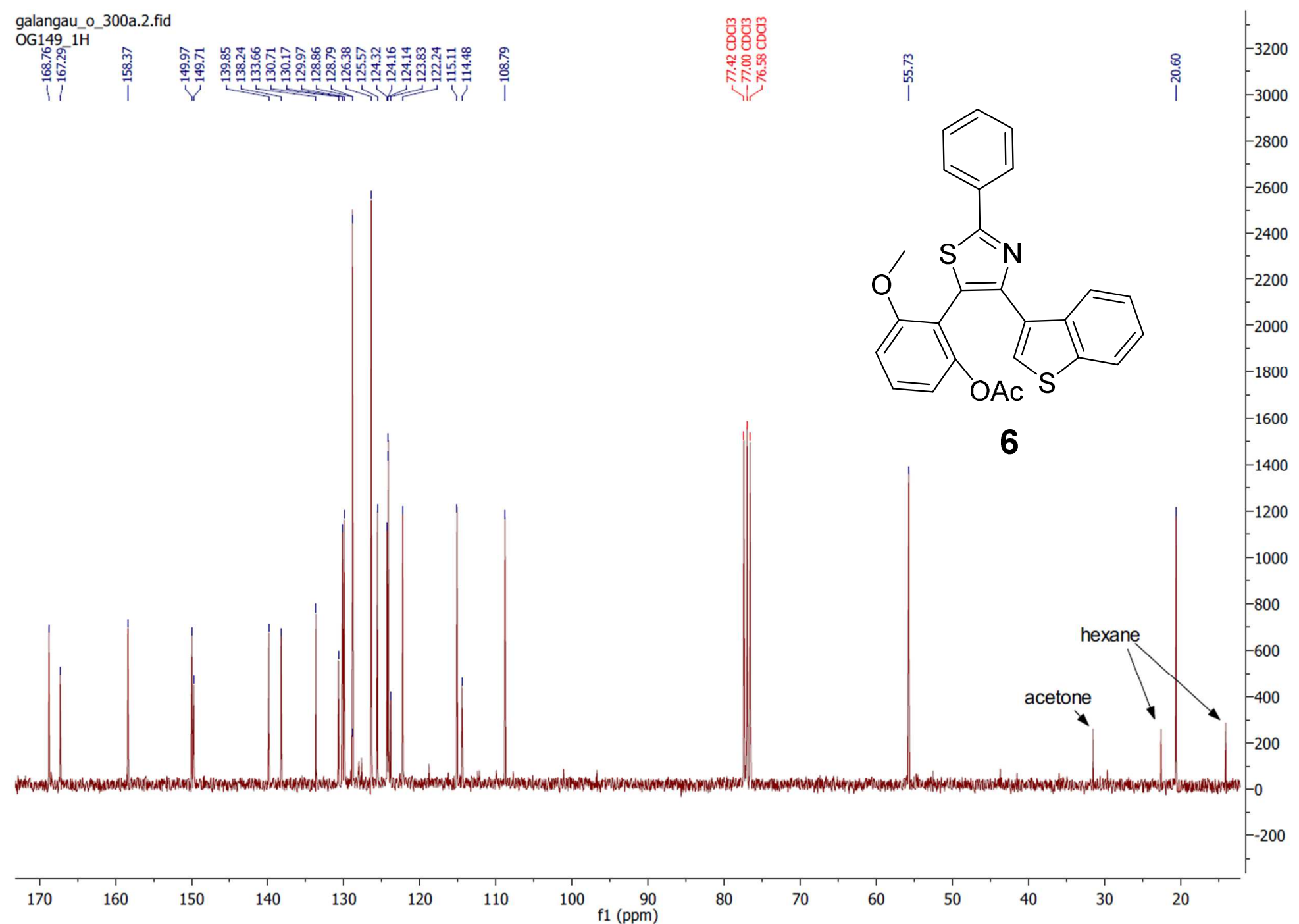

Figure $\mathrm{S}^{10} 0^{13} \mathrm{C}-\mathrm{NMR}$ of compound 6 in $\mathrm{CDCl}_{3}$ 


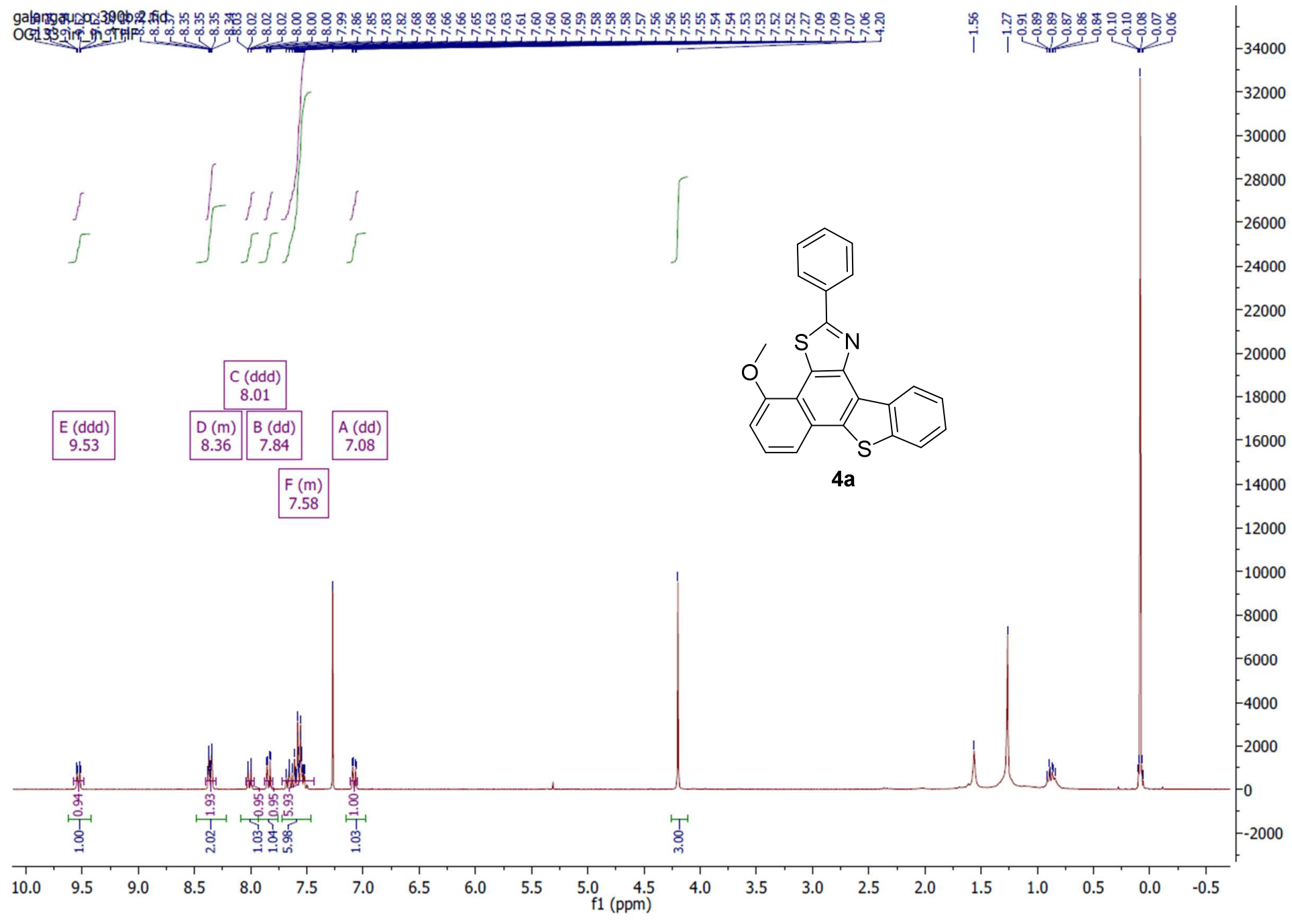

Figure $\mathrm{S}^{1}{ }^{1} \mathrm{H}-\mathrm{NMR}$ of compound $4 \mathrm{a}$ in $\mathrm{CDCl}_{3}$ 


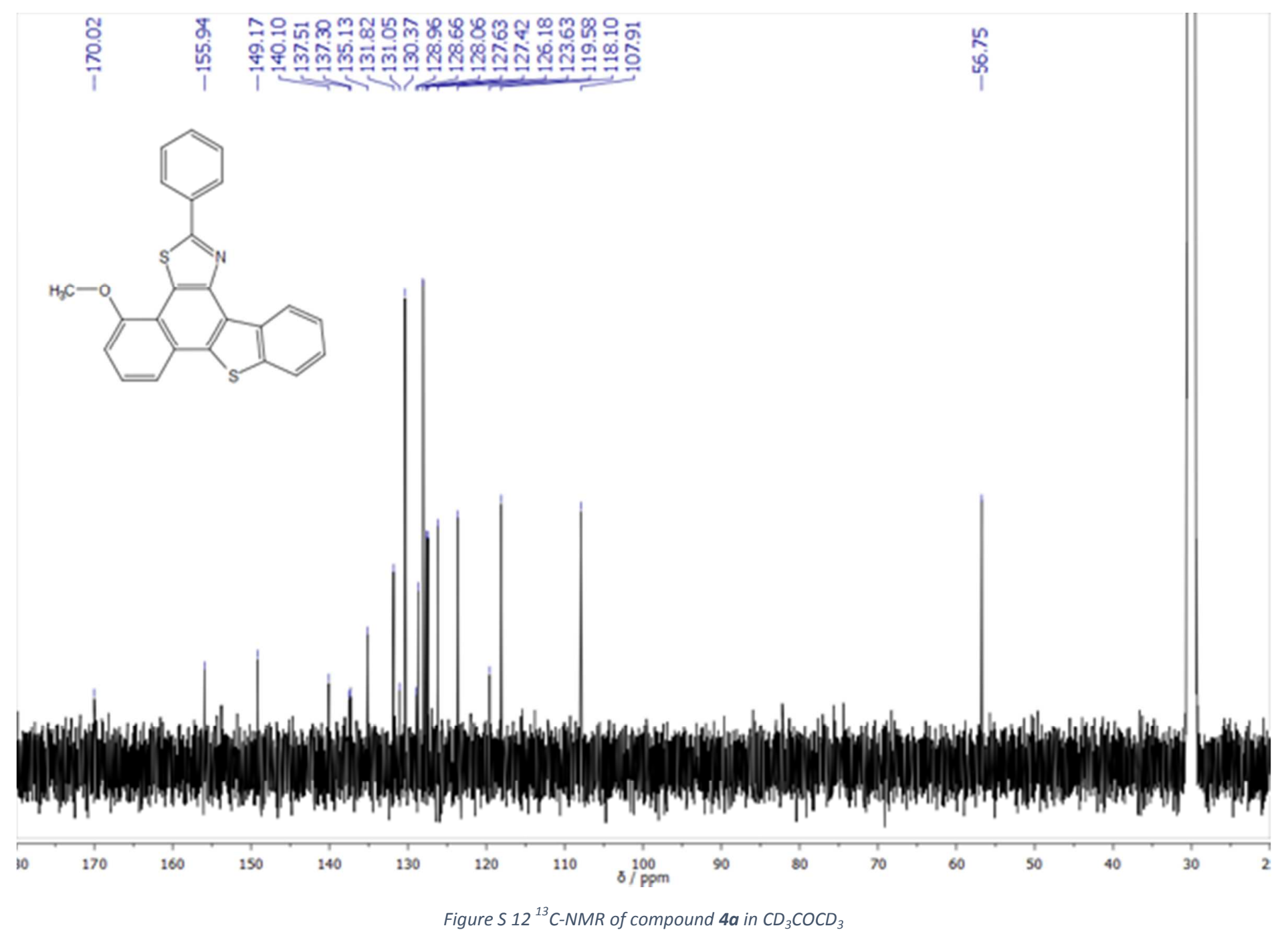




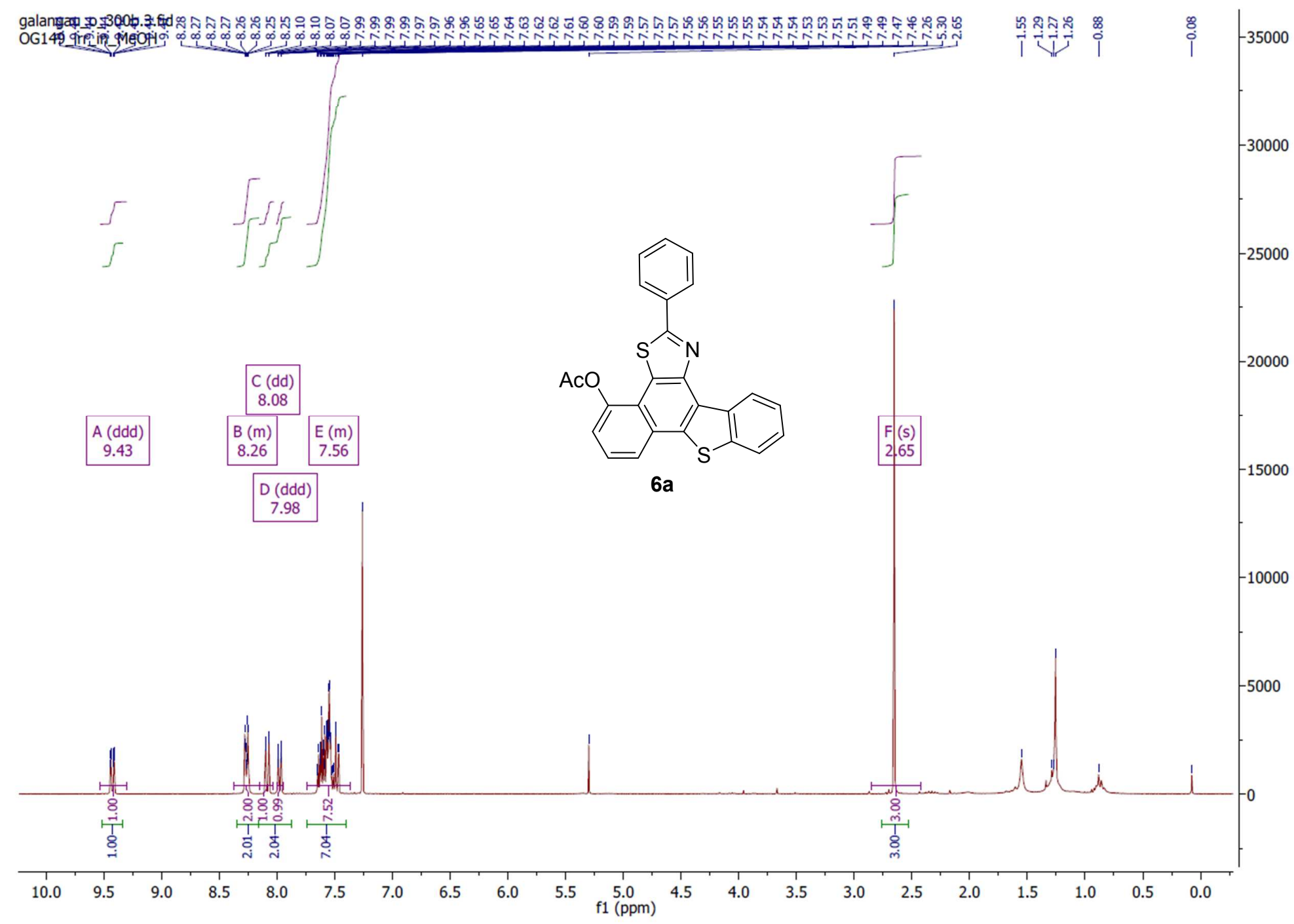

Figure $\mathrm{S}_{13}{ }^{1} \mathrm{H}$-NMR of compound $6 \mathrm{a}$ in $\mathrm{CDCl}_{3}$ 


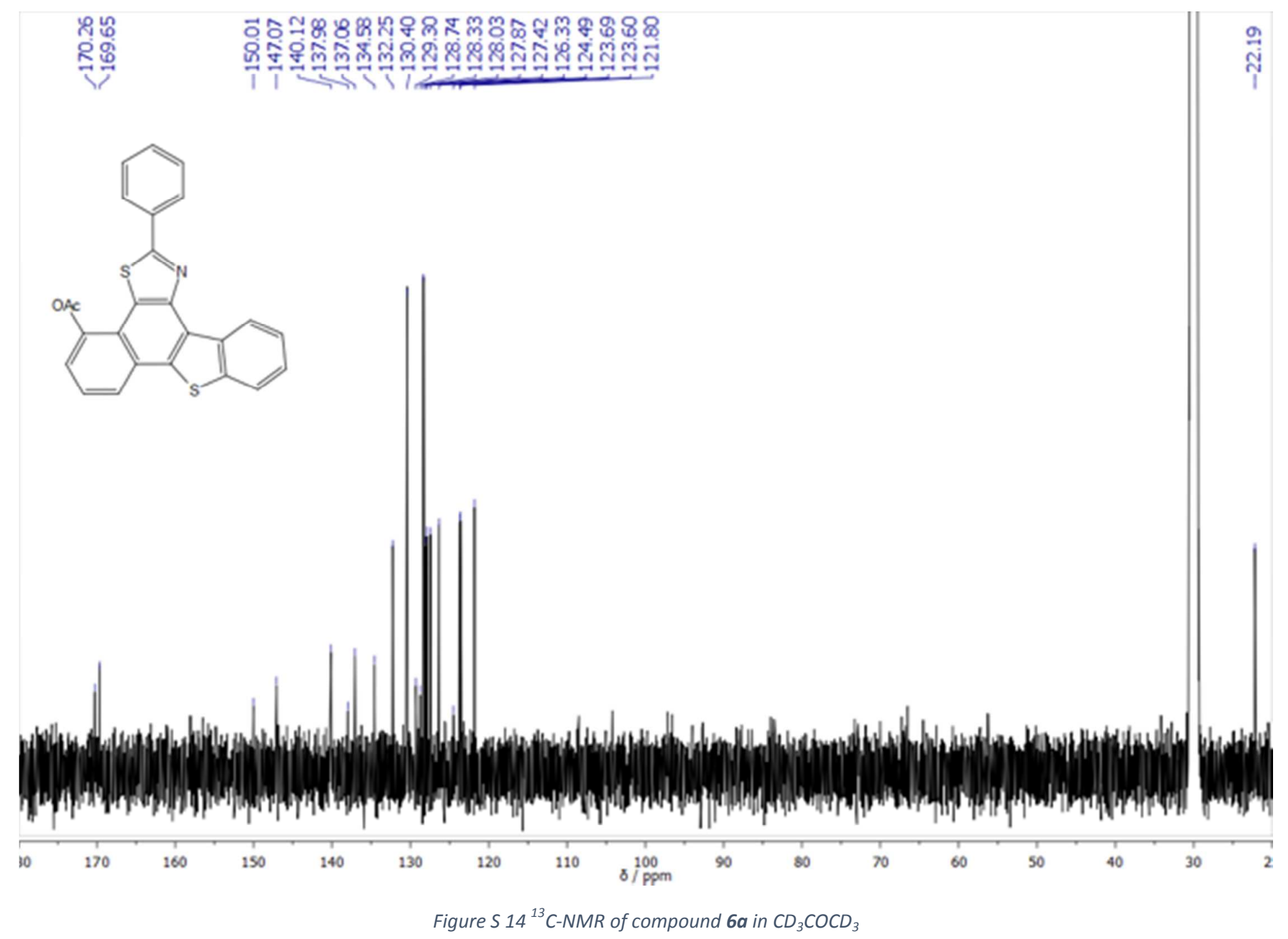


VT-NMR of compound 40 in $\mathrm{MeOH}$

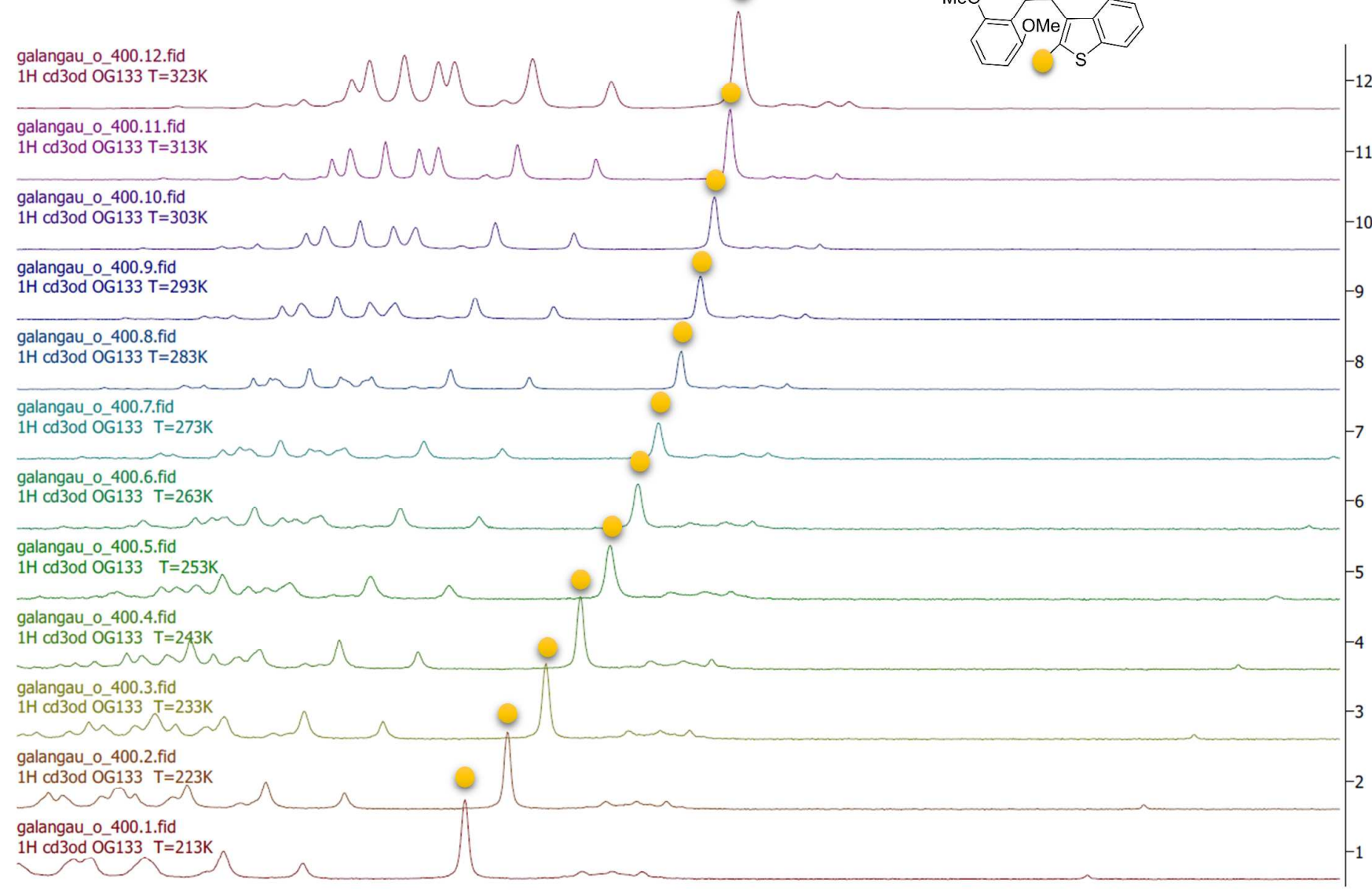

7.43 7.42 7.41 7.40 7.39 7.38 7.37 7.36 7.35 7.34 7.33 7.32 7.31 7.30 7.29 7.28 7.27 7.26 7.25 7.24 7.23 7.22 7.21 7.20 7.19 7.18 7.17 7.16 7.15 7.14 7.13 7.12 7.11 7.10 7.09 f1 $(\mathrm{ppm})$

Figure S15 VT-NMR of compound 40 in $C D_{3} O D$ 


\section{UV-Visible experiments}

\section{Photolysis experiments on compound 4}
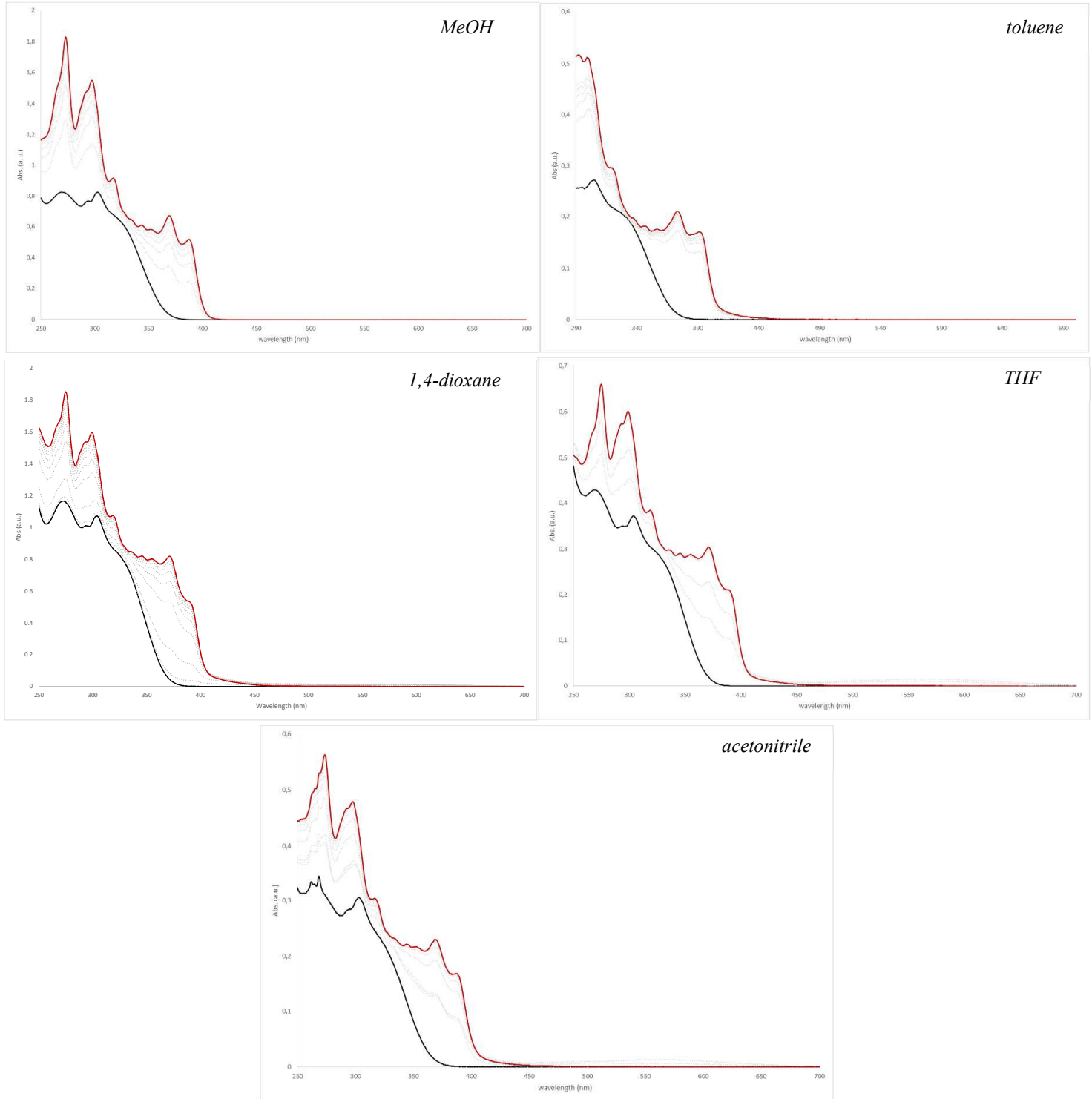

Figure S16 Photolysis of compound $\mathbf{4}$ in different solvent, at room temperature. black line: open form; red line: photostationary state 


\section{Photolysis experiments on compound 6}
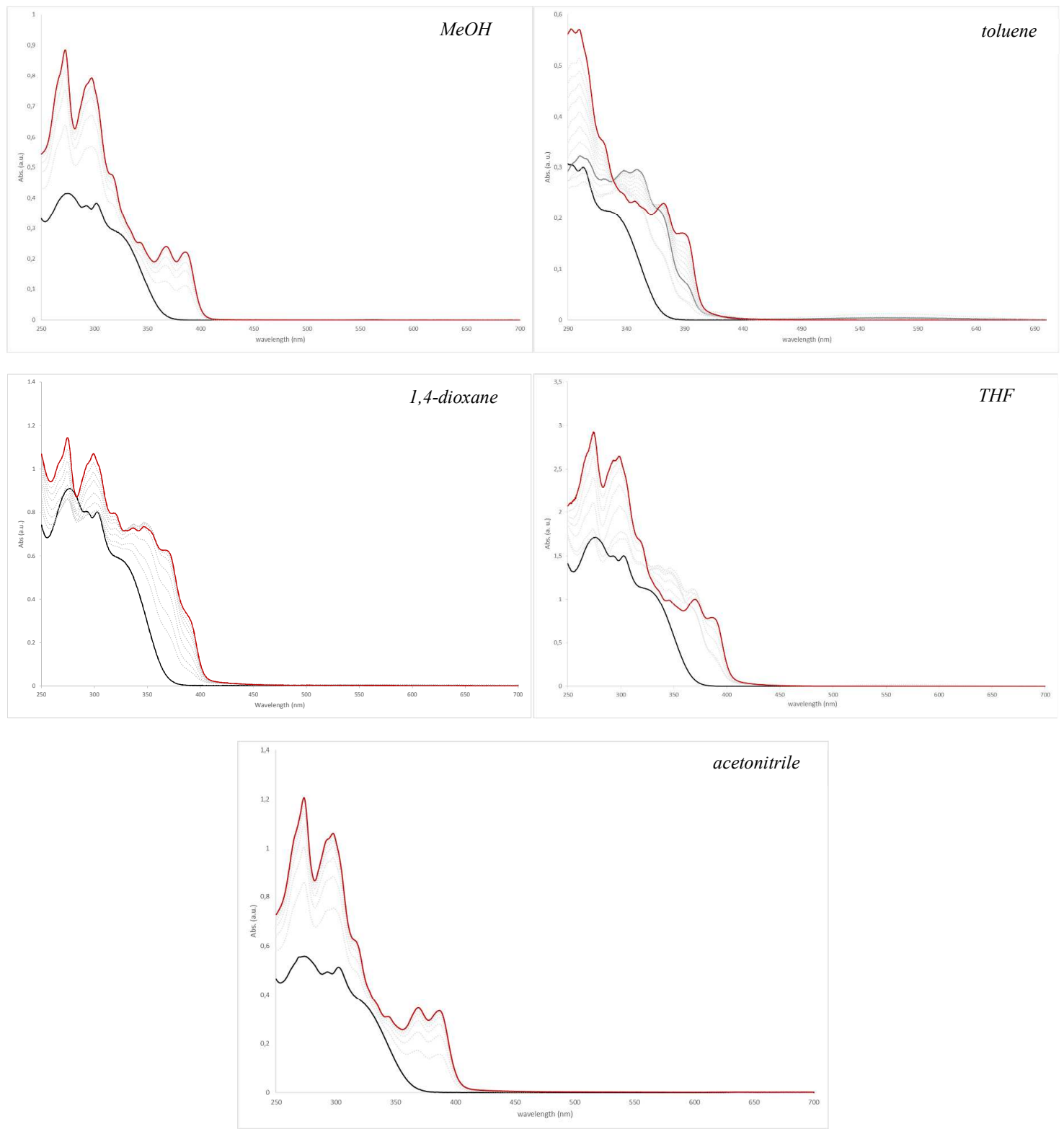

Figure S17 Photolysis of compound 6 in different solvent, at room temperature. black line: open form; red line: photostationary state 
Absorption spectra of compounds $4 a$ and $6 a$ in $\mathrm{THF}$

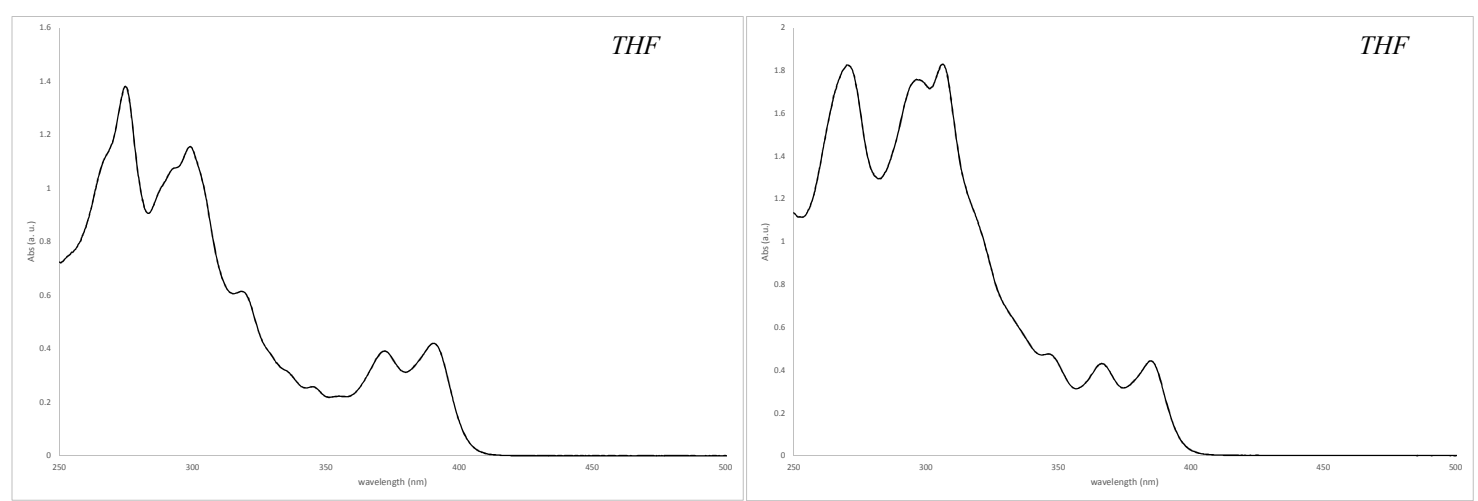

Figure S18 Comparative absorption spectra of compounds $4 a$ (left) and $6 a$ (right) in THF 


\section{Monitoring of the photo-reactions by NMR experiments}

\section{Photolysis experiments on compound 60}

NMR spectra were recorded on Avance Bruker 500 spectrometer $\left({ }^{1} \mathrm{H}, 500 \mathrm{MHz}\right)$ equipped with TXI probe, using standard sequences. Data sets were processed using Bruker Topspin 3.2 software. Samples (ca 1mM) are dissolved in 11 deuterated solvents in NMR tubes. Photoirradiation was carried out directly into the NMR tube with a Bioblock Scientific VL-6LC lamp (12 W) operating at $312 \mathrm{~nm}$.

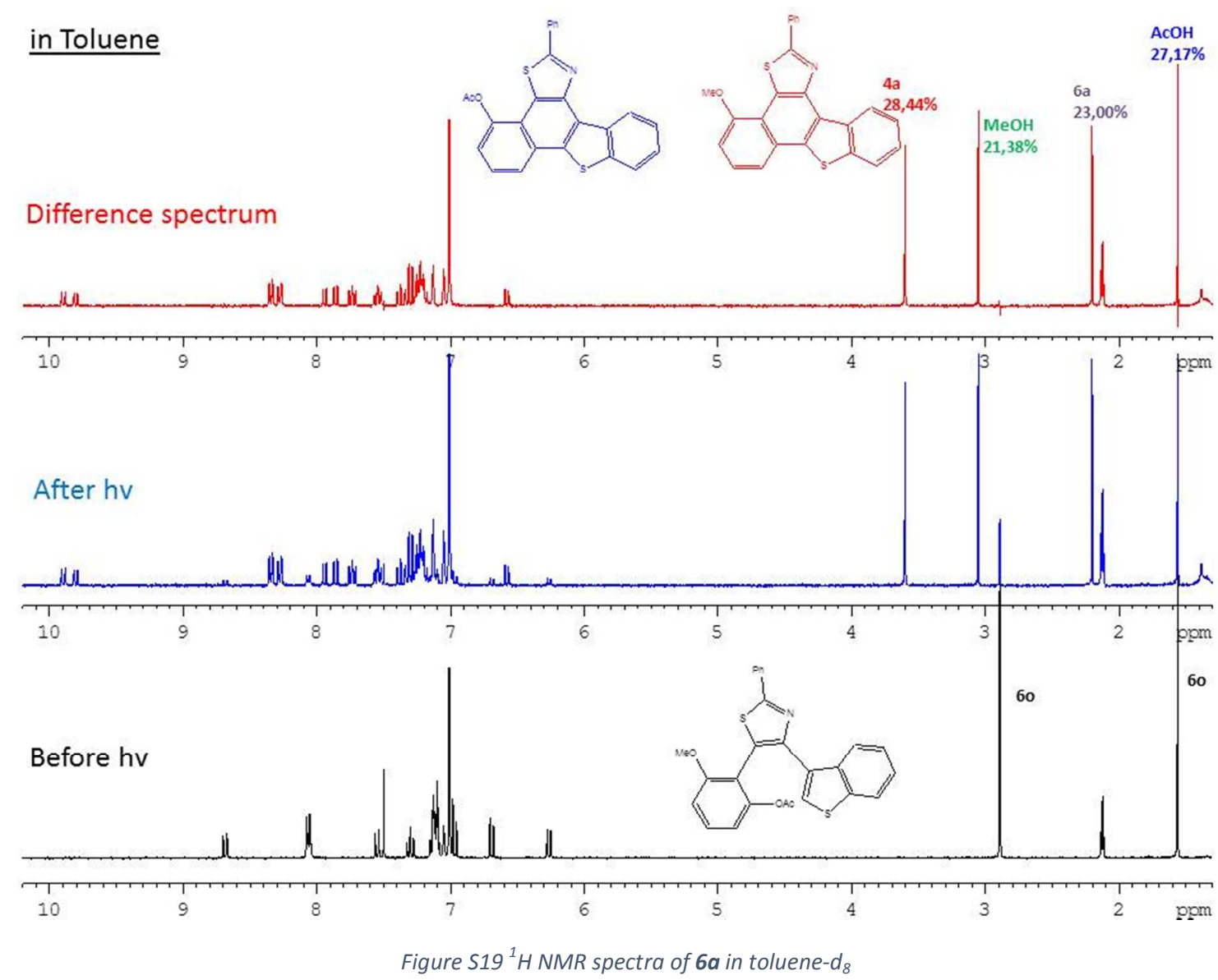




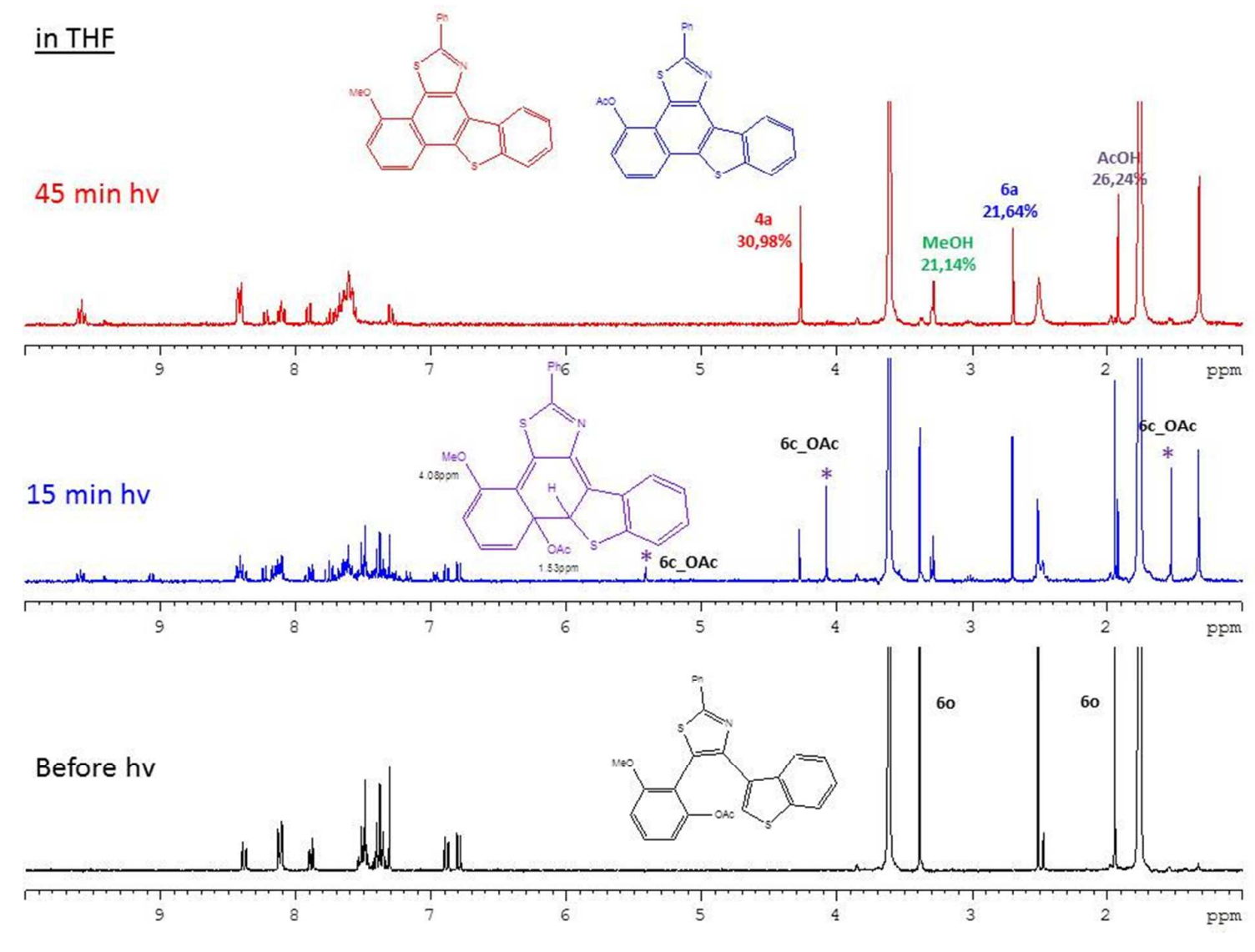

Figure $52 O^{1} H$ NMR spectra of $6 a$ in tetrahydrofurane- $d_{8}$ 


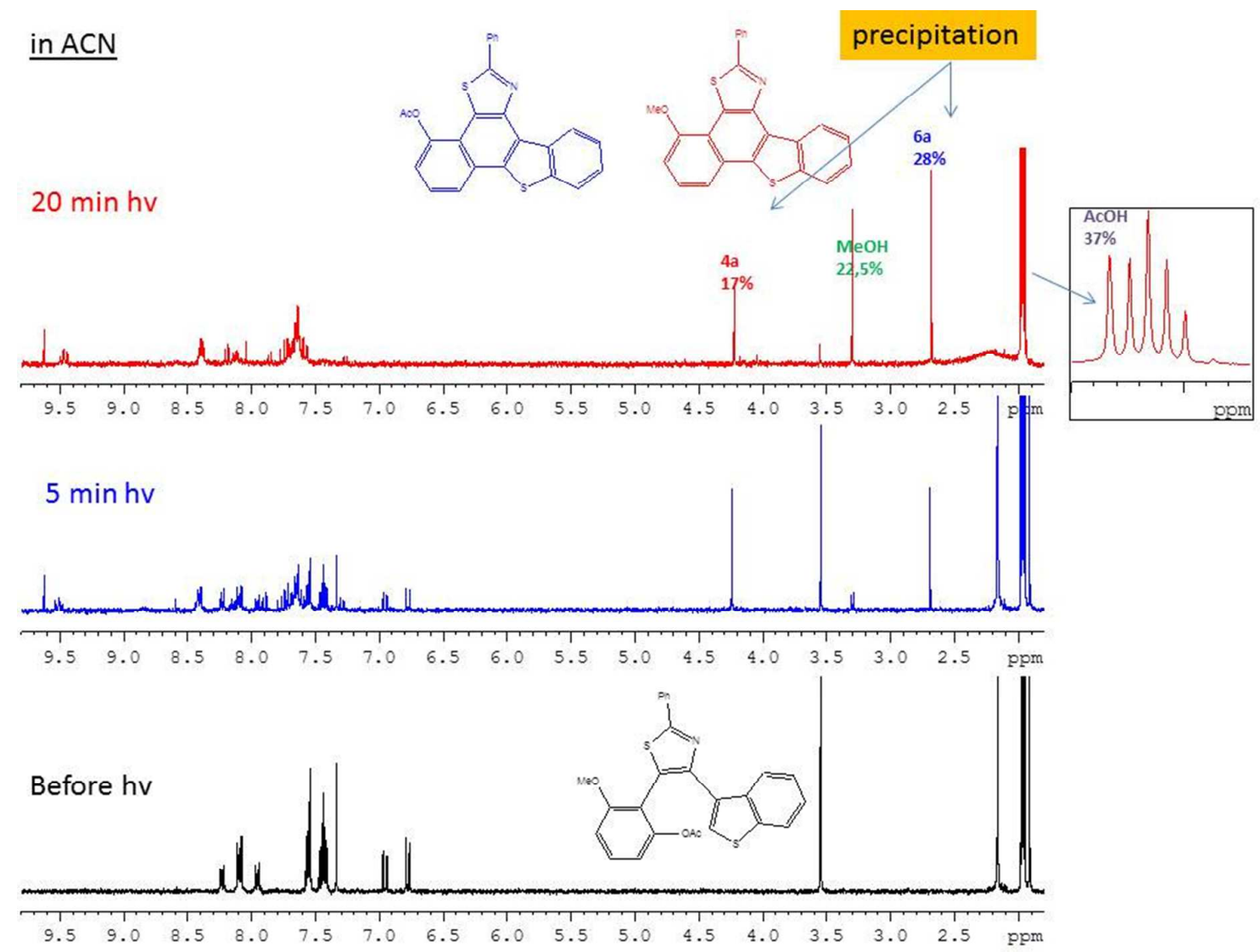

Figure $S 21^{1} \mathrm{H}$ NMR spectra of $6 \boldsymbol{a}$ in acetonitrile- $d_{3}$ 


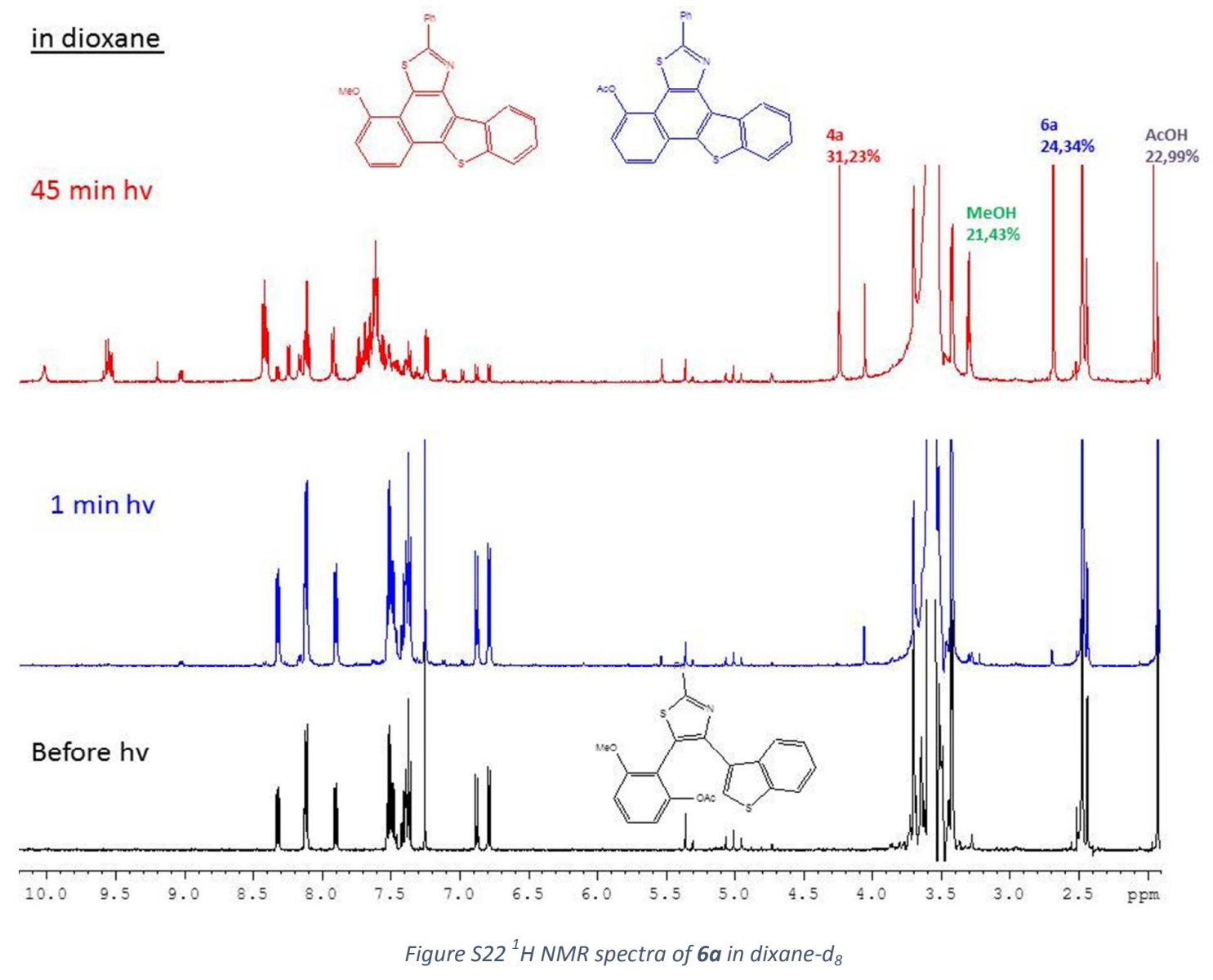




\section{in $\mathrm{CDCl} 3$}
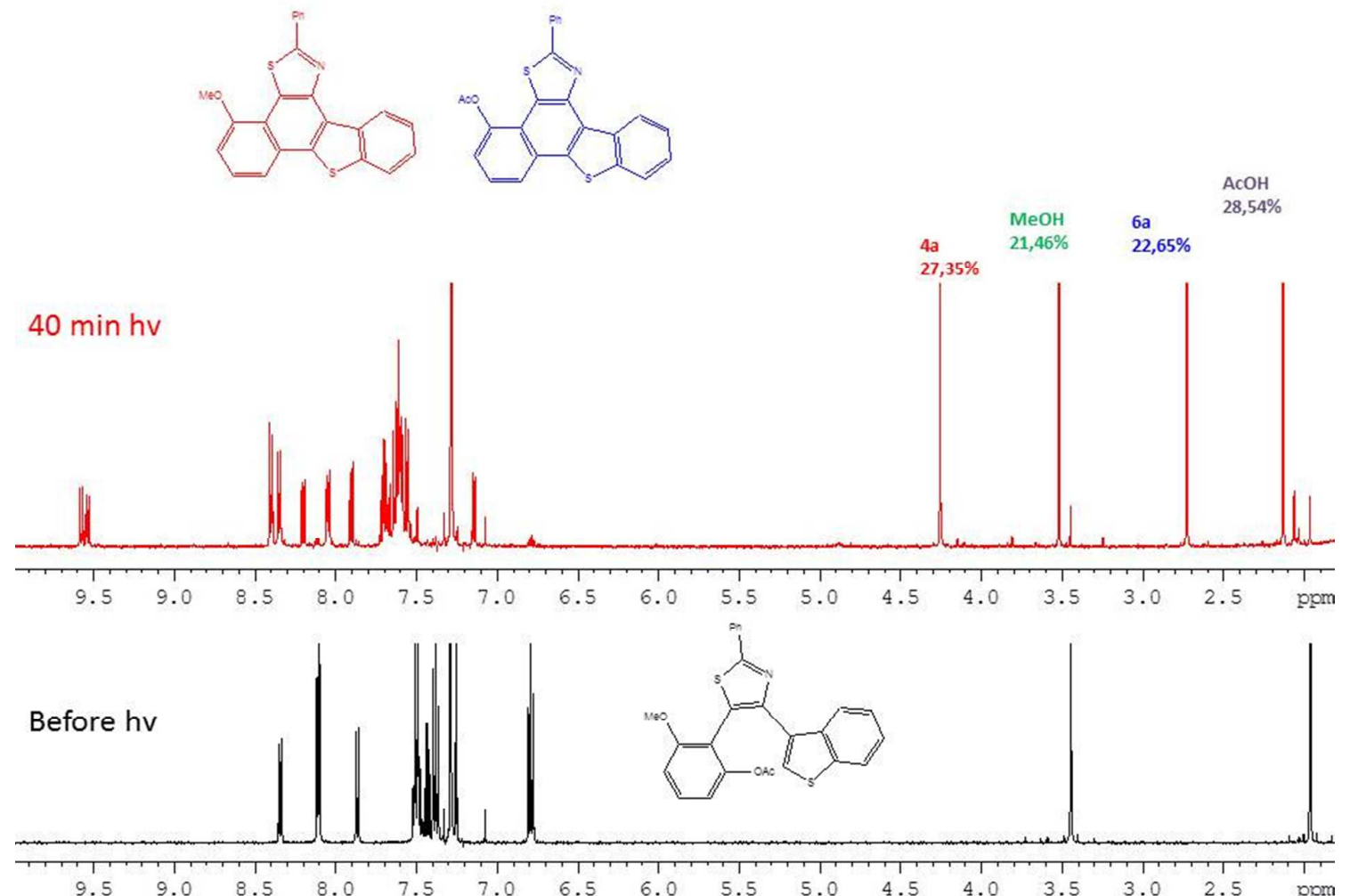

Figure $S 23{ }^{1}$ H NMR spectra of 6 a in chloroform- $d_{1}$ 
in DMSO
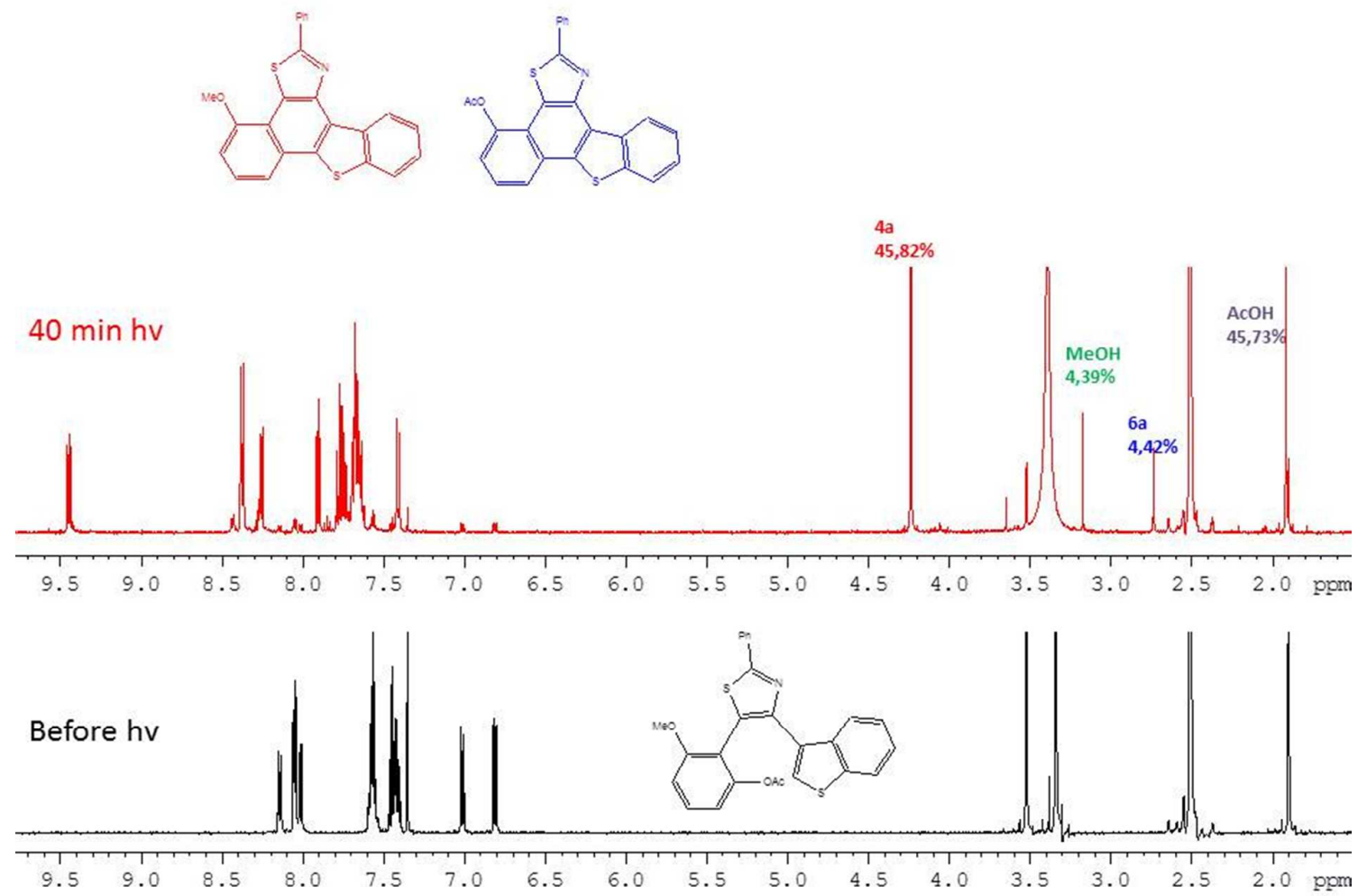

Figure S24 ${ }^{1} H$ NMR spectra of $6 a$ in DMSO- $d_{6}$ 
in DCM
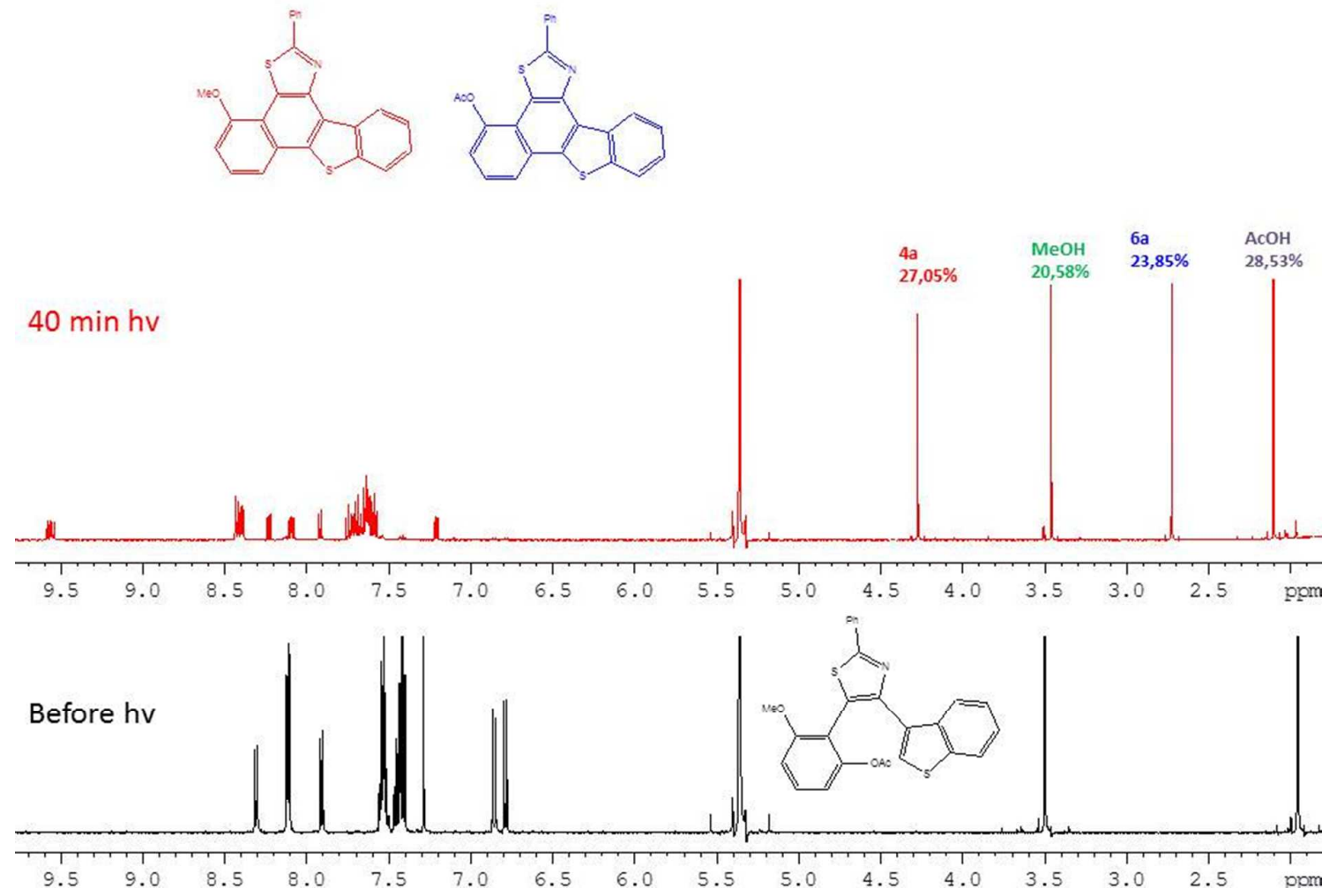

Figure $S 25{ }^{1} \mathrm{H}$ NMR spectra of $6 a$ in methylene chloride- $d_{2}$ 
$\underline{\text { in DMF }}$
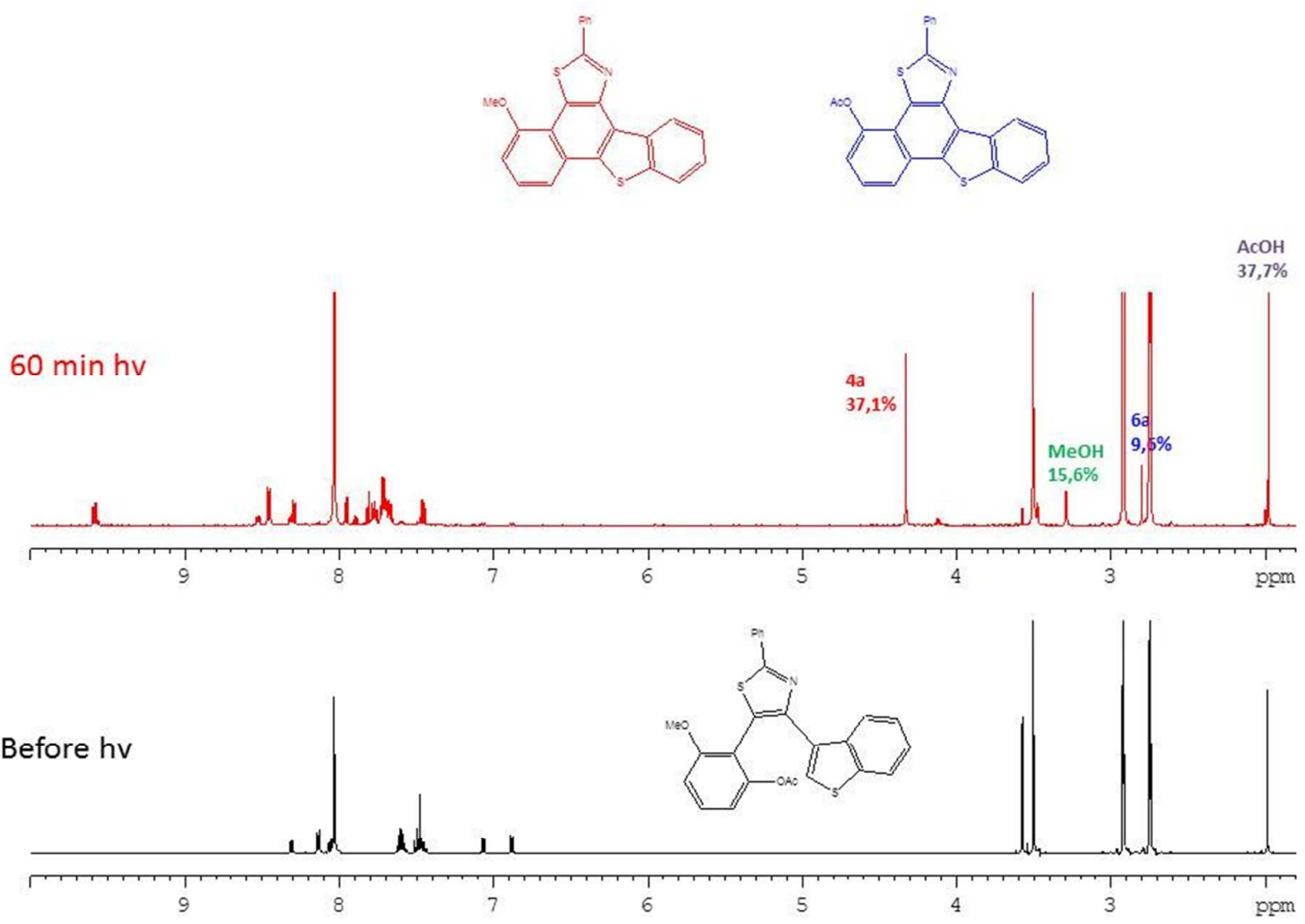

Figure $S 26{ }^{1} H$ NMR spectra of 6 a in dimethyl-formamide- $d_{7}$ 


\section{in EtOD}

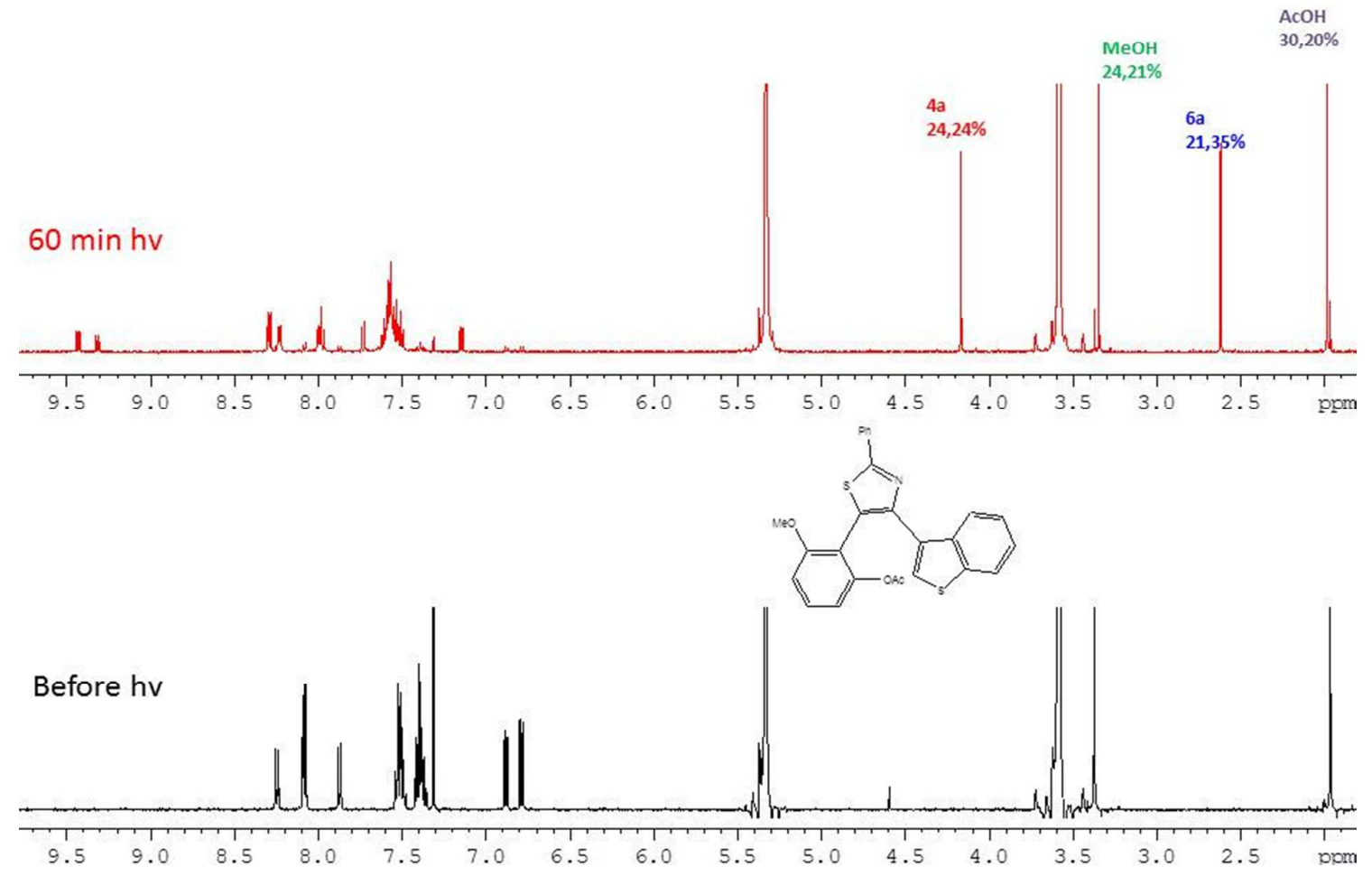

Figure $S 27^{1} H$ NMR spectra of $6 a$ in ethnaol- $d_{6}$ 

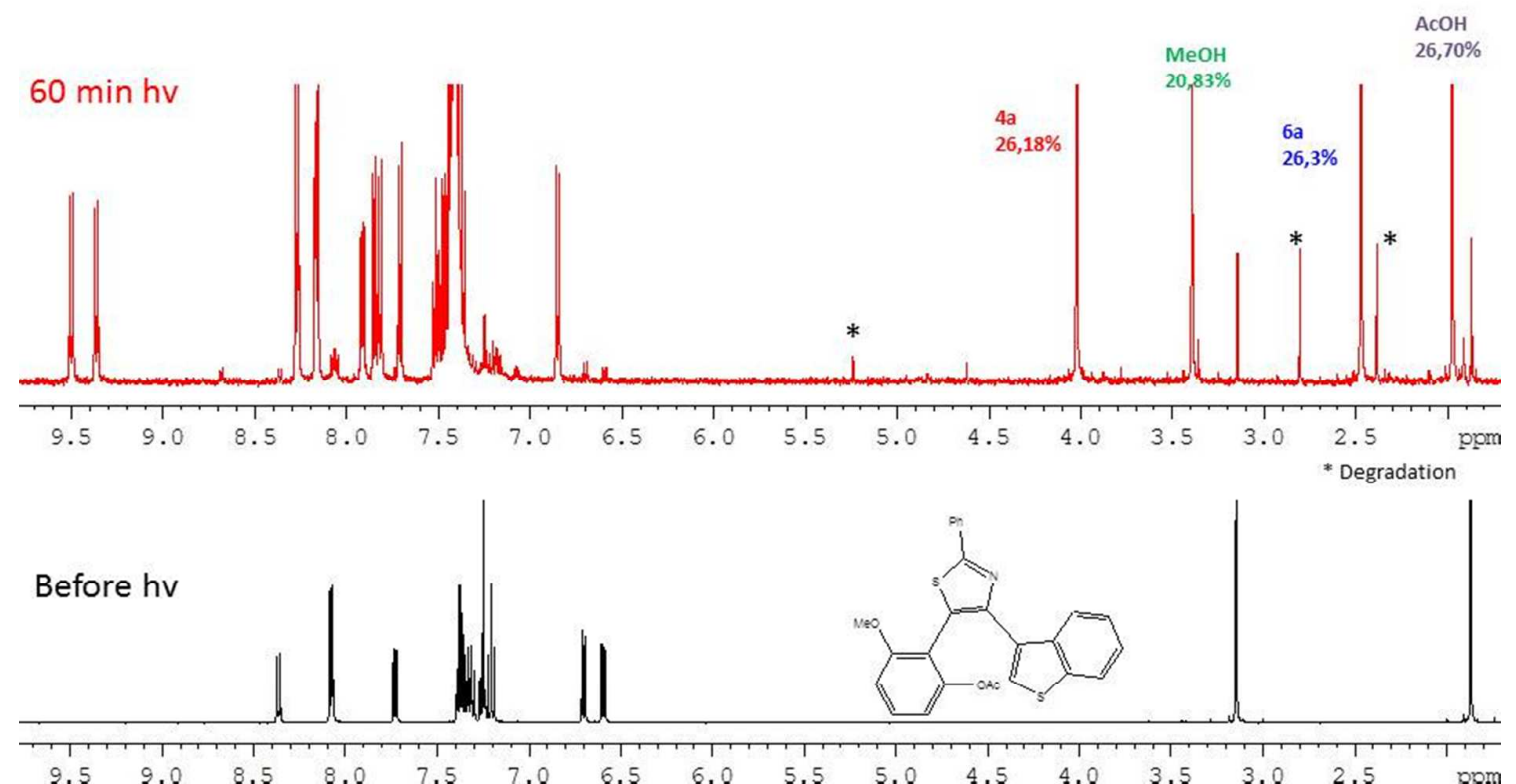

Figure $S 28{ }^{1}$ H NMR spectra of $6 a$ in cyclohexane- $d_{12}$ 


\section{Characterization of $6 c_{-} O A c$}

1 and 2D NMR spectra were recorded on Avance Bruker 500 spectrometer in THF- $\mathrm{d}_{8}$ at $-60^{\circ} \mathrm{C}$

Characterization of $6 \mathrm{c}-\mathrm{OAC}$

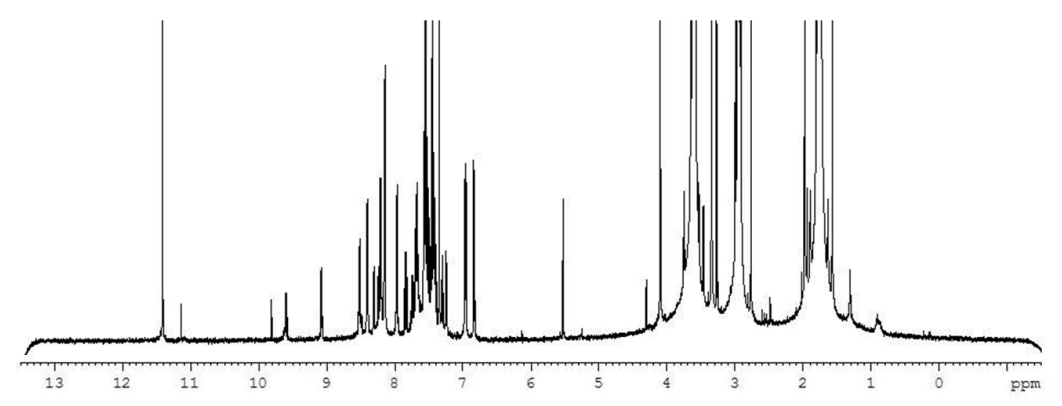

Figure S29 1H NMR spectrum

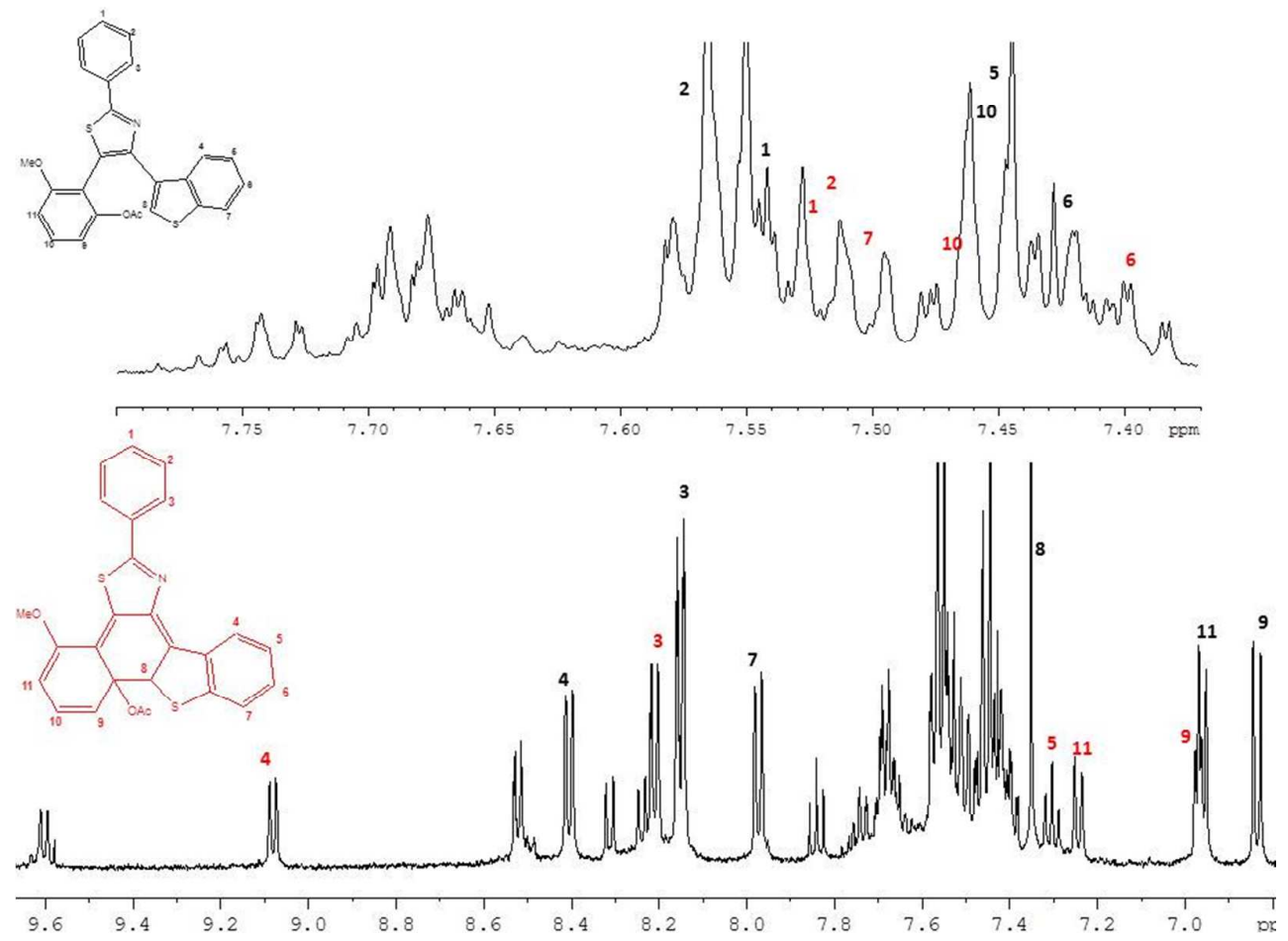

Figure $S 30^{1} \mathrm{H} N M R$ spectrum - Aromatic part 

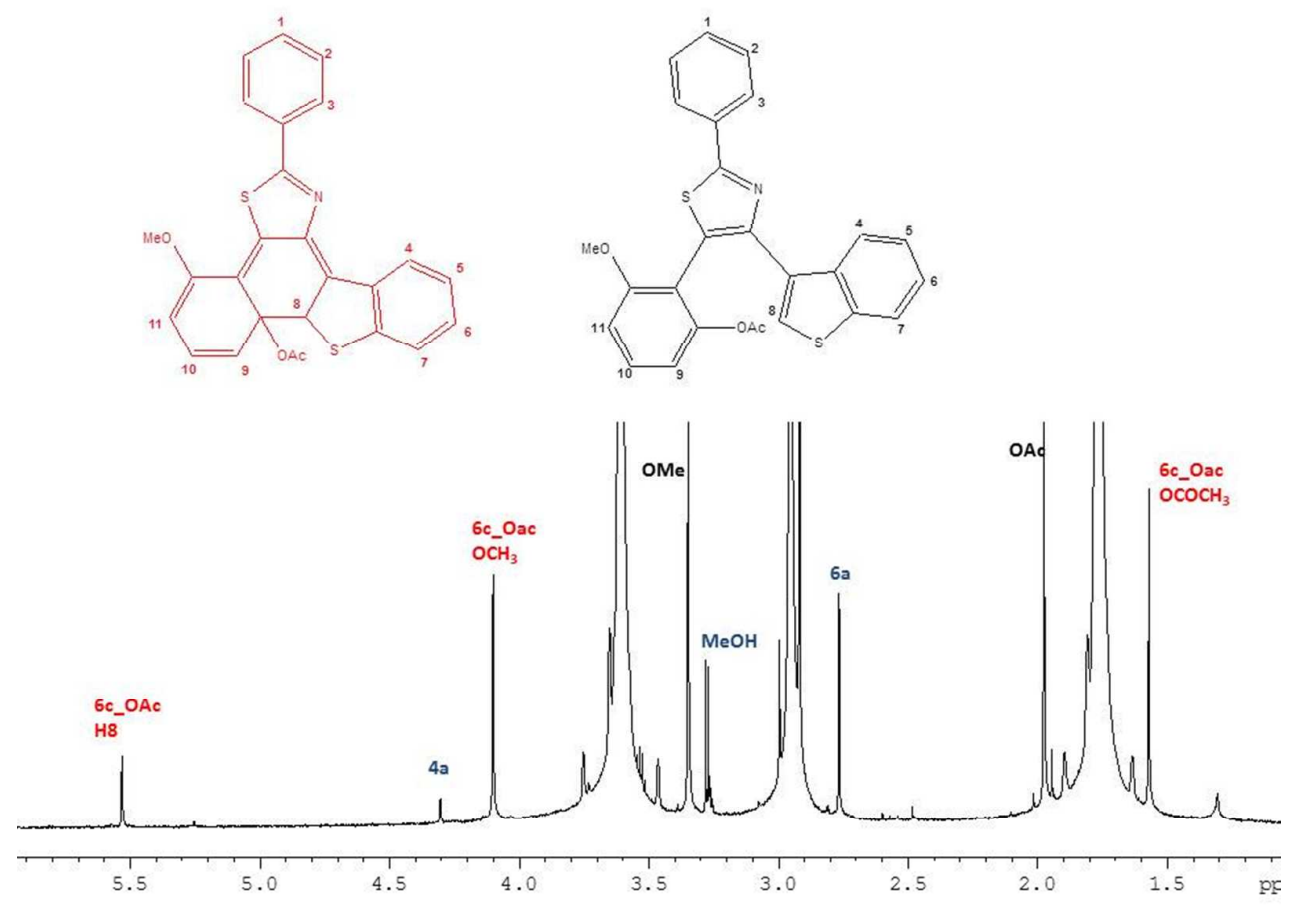

Figure S31 1H NMR spectrum - Aliphatic part

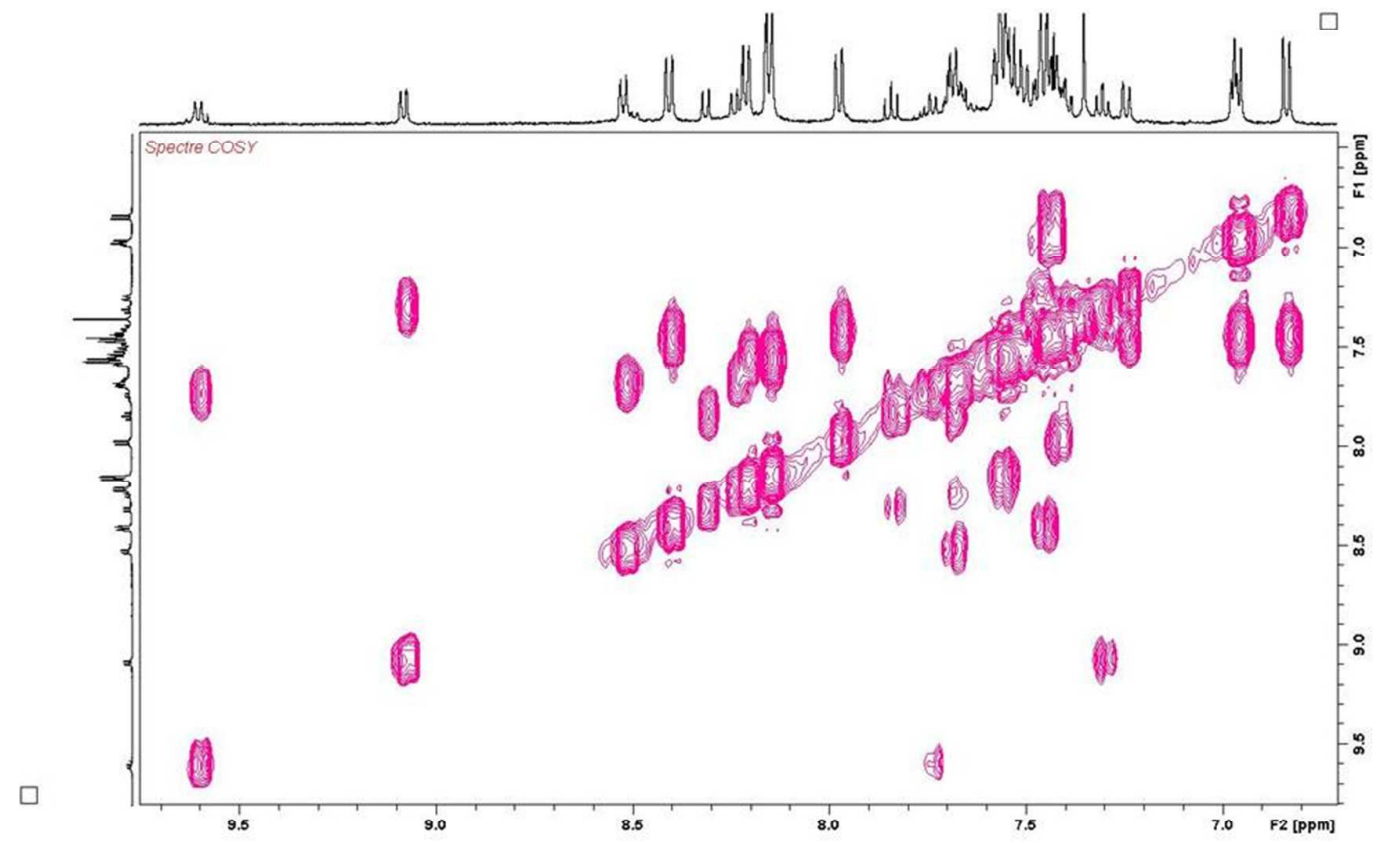

Figure $532{ }^{1} \mathrm{H}-1 \mathrm{H}$ COSY NMR spectrum - Aromatic part 


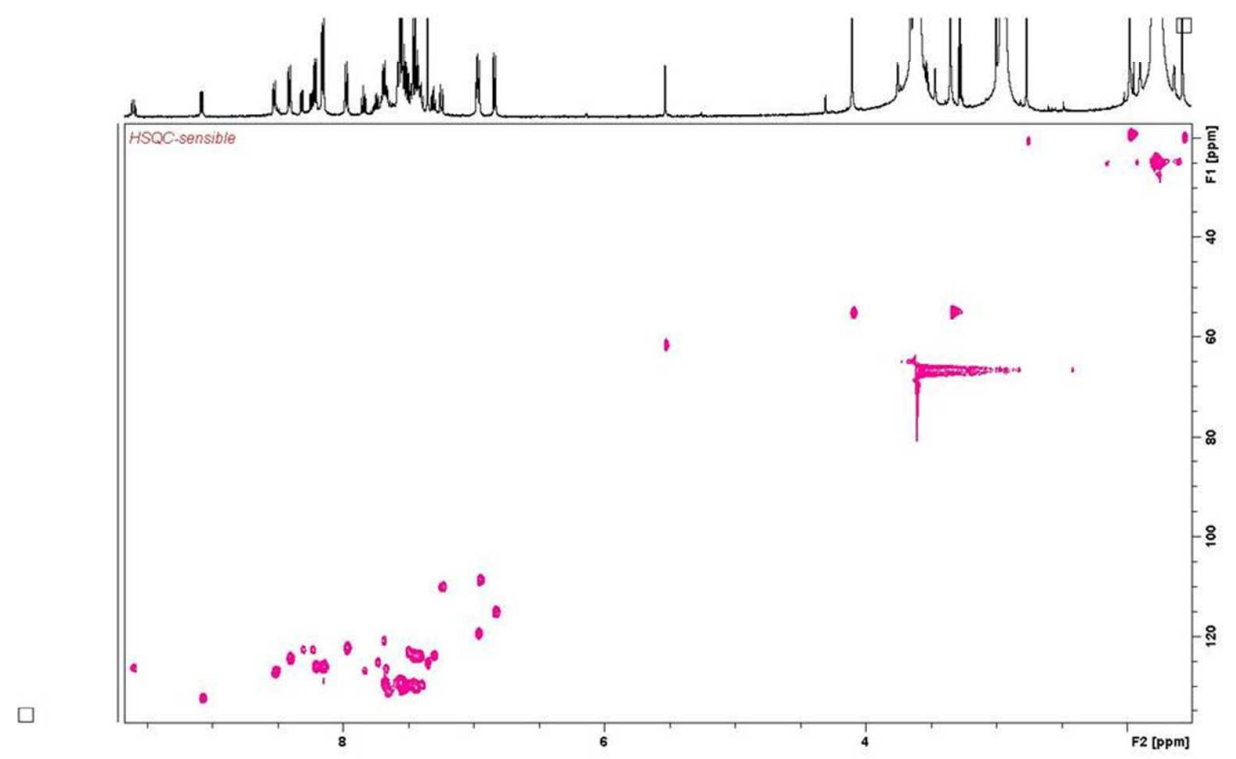

Figure $533{ }^{1} \mathrm{H}-13 \mathrm{C}$ HSQC NMR spectrum

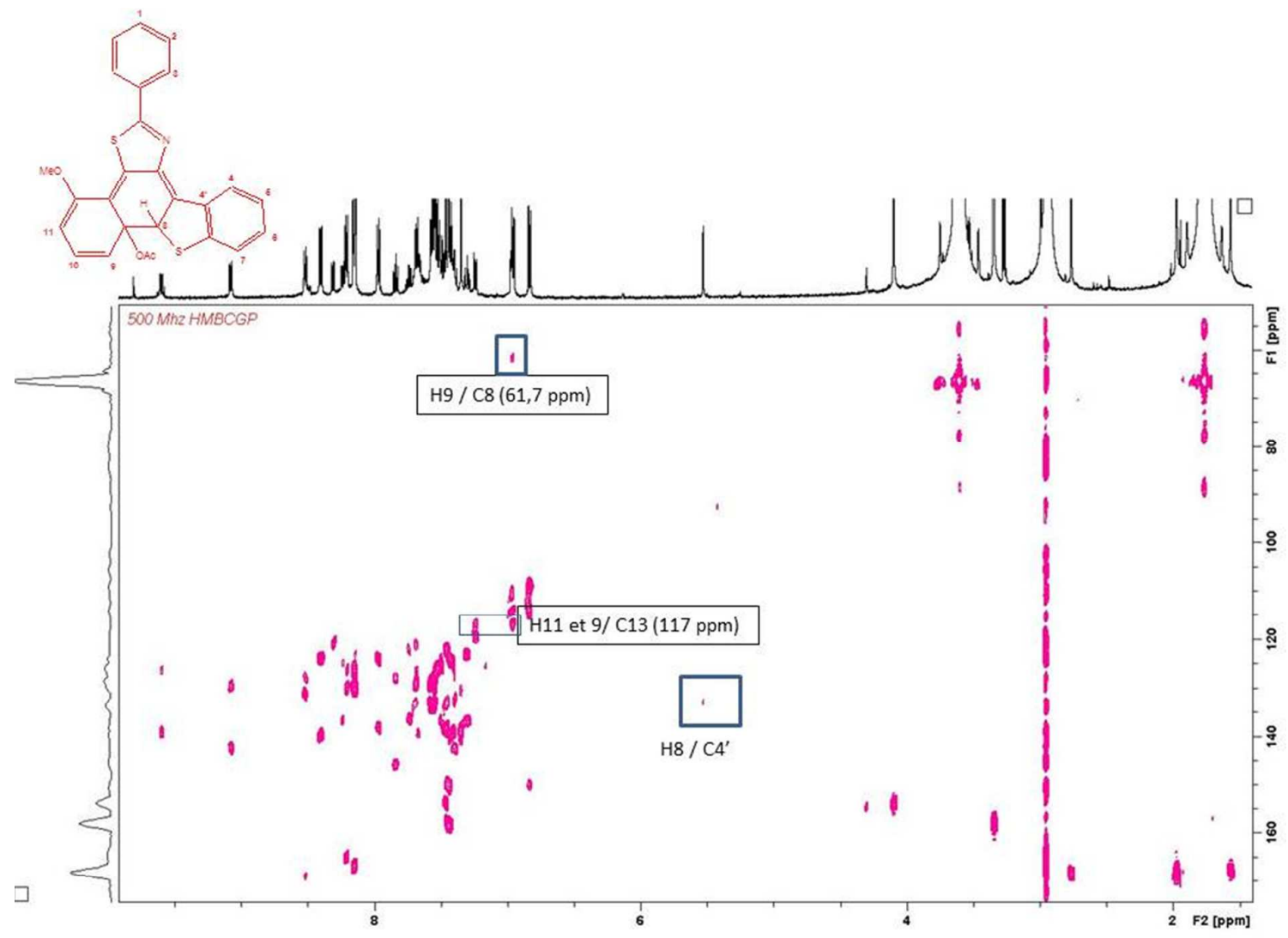

Figure $534{ }^{1} \mathrm{H}^{13} \mathrm{C}$ long-range correlation NMR spectrum 


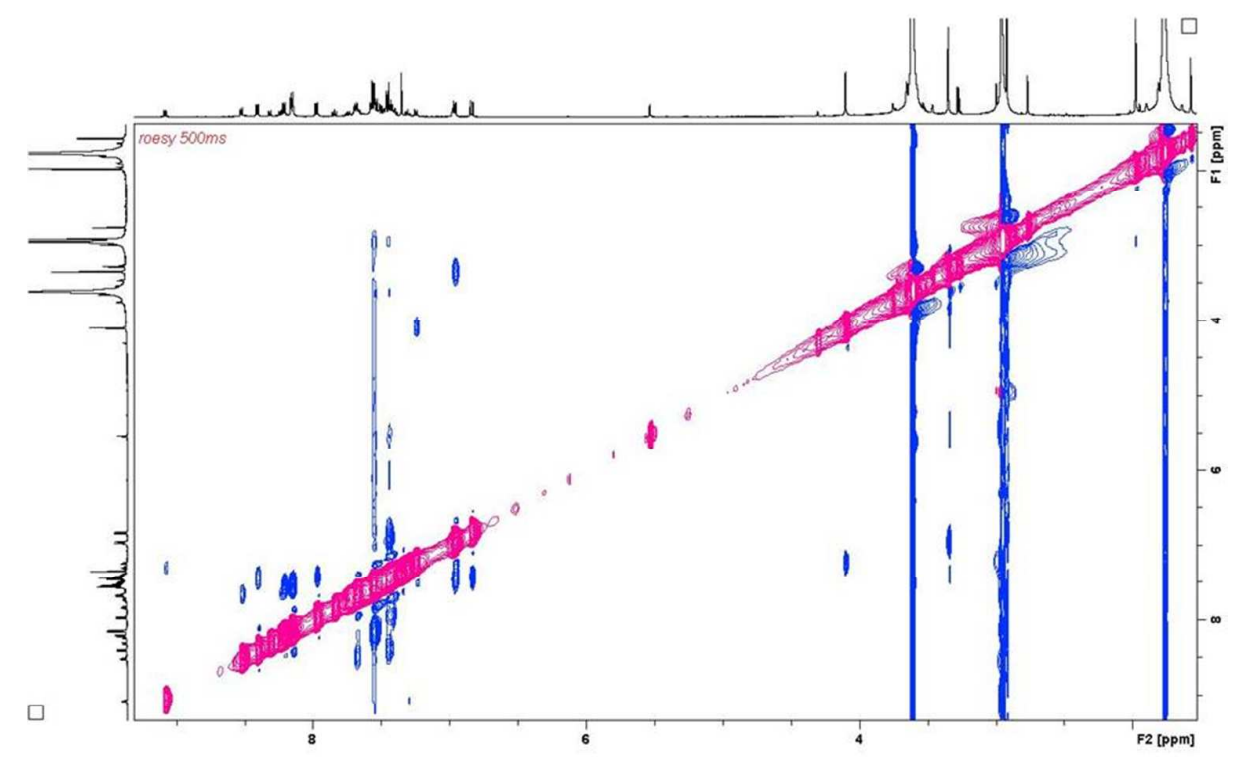

Figure $S 35{ }^{1} \mathrm{H}-{ }^{1} \mathrm{H}$ ROESY NMR spectrum

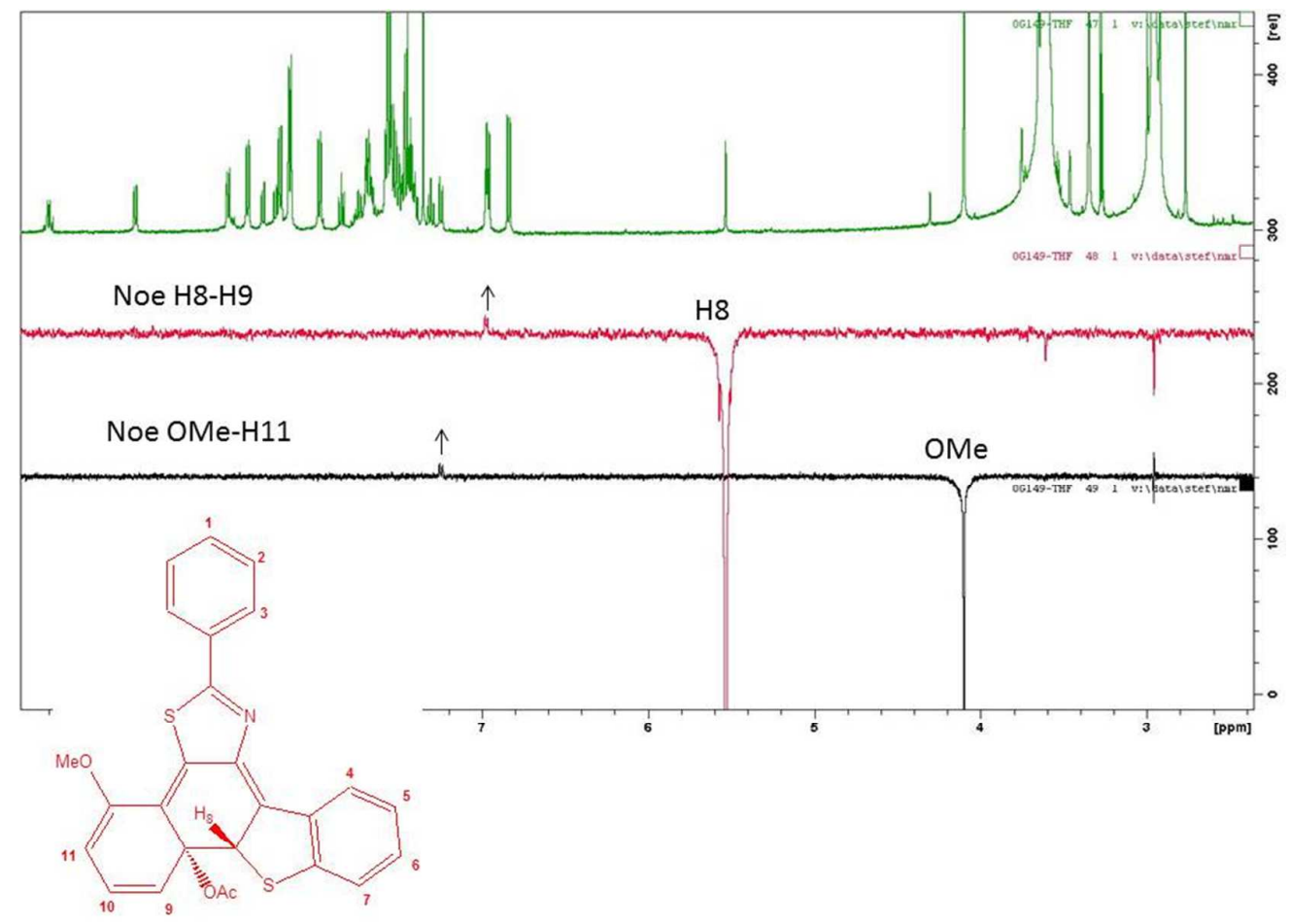

Figure S36 1D ROESY NMR spectra 


\section{Computational details}

\section{Gaussian Reference:}

Gaussian 09, Revision C.01, M. J. Frisch, G. W. Trucks, H. B. Schlegel, G. E. Scuseria, M. A. Robb, J. R. Cheeseman, G. Scalmani, V. Barone, B. Mennucci, G. A. Petersson, H. Nakatsuji, M. Caricato, X. Li, H. P. Hratchian, A. F. Izmaylov, J. Bloino, G. Zheng, J. L. Sonnenberg, M. Hada, M. Ehara, K. Toyota, R. Fukuda, J. Hasegawa, M. Ishida, T. Nakajima, Y. Honda, O. Kitao, H. Nakai, T. Vreven, J. A. Montgomery, Jr., J. E. Peralta, F. Ogliaro, M. Bearpark, J. J. Heyd, E. Brothers, K. N. Kudin, V. N. Staroverov, T. Keith, R. Kobayashi, J. Normand, K. Raghavachari, A. Rendell, J. C. Burant, S. S. Iyengar, J. Tomasi, M. Cossi, N. Rega, J. M. Millam, M. Klene, J. E. Knox, J. B. Cross, V. Bakken, C. Adamo, J. Jaramillo, R. Gomperts, R. E. Stratmann, O. Yazyev, A. J. Austin, R. Cammi, C. Pomelli, J. W. Ochterski, R. L. Martin, K. Morokuma, V. G. Zakrzewski, G. A. Voth, P. Salvador, J. J. Dannenberg, S. Dapprich, A. D. Daniels, O. Farkas, J. B. Foresman, J. V. Ortiz, J. Cioslowski, and D. J. Fox, Gaussian, Inc., Wallingford CT, 2010. 


\section{Details on compound 4}

Corresponding Z-matrix of compound 40

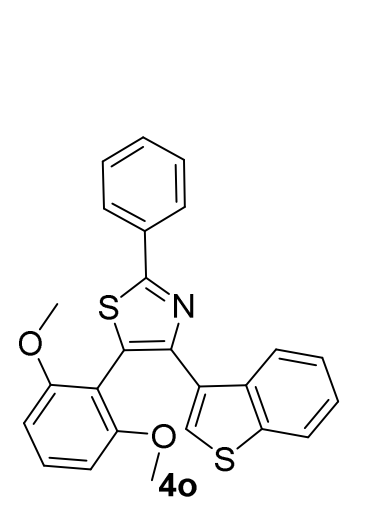

\begin{tabular}{|c|c|c|c|}
\hline \multirow{2}{*}{$\begin{array}{l}\text { Center } \\
\text { Number }\end{array}$} & \multicolumn{3}{|c|}{ Coordinates (Angstroms) } \\
\hline & 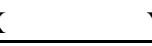 & 2 & \\
\hline 1 & 3.011002 & -3.934332 & -0.429761 \\
\hline 2 & 2.484545 & -2.701361 & -0.824134 \\
\hline 3 & 1.246235 & -2.239421 & -0.341815 \\
\hline 4 & 0.524741 & -3.03522 & 0.560662 \\
\hline 5 & 1.04512 & -4.254847 & 0.956083 \\
\hline 6 & 2.28149 & -4.703309 & 0.462421 \\
\hline 7 & 3.22071 & -1.531774 & -1.896699 \\
\hline 8 & 1.882309 & -0.442356 & -1.676826 \\
\hline 9 & 0.923523 & -0.919514 & -0.846662 \\
\hline 10 & -0.259399 & -0.153736 & -0.42219 \\
\hline 11 & -0.211474 & 1.114116 & 0.09982 \\
\hline 12 & -1.822926 & 1.615819 & 0.49698 \\
\hline 13 & -2.431287 & 0.07756 & -0.051505 \\
\hline 14 & -1.505679 & -0.726446 & -0.495173 \\
\hline 15 & -3.860893 & -0.274558 & -0.012408 \\
\hline 16 & -4.841594 & 0.656267 & 0.344955 \\
\hline 17 & -6.181469 & 0.286582 & 0.383087 \\
\hline 18 & -6.557466 & -1.01574 & 0.064385 \\
\hline 19 & -5.585307 & -1.947175 & -0.2955 \\
\hline 20 & -4.245785 & -1.581919 & -0.33451 \\
\hline 21 & 3.967046 & -4.279656 & -0.809102 \\
\hline 22 & -0.43094 & -2.685601 & 0.936241 \\
\hline 23 & 0.49174 & -4.87482 & 1.653815 \\
\hline 24 & 2.673145 & -5.662695 & 0.784425 \\
\hline 25 & -4.566631 & 1.678452 & 0.589539 \\
\hline 26 & -6.932033 & 1.018558 & 0.661967 \\
\hline 27 & -5.870815 & -2.96427 & -0.543832 \\
\hline 28 & -3.482697 & -2.300166 & -0.612457 \\
\hline 29 & 1.888845 & 0.517812 & -2.174294 \\
\hline 30 & 0.985671 & 1.915187 & 0.40559 \\
\hline 31 & 1.172049 & 3.194315 & -0.145873 \\
\hline 32 & 1.996646 & 1.3753 & 1.222734 \\
\hline 33 & 2.347982 & 3.910124 & 0.090657 \\
\hline 34 & 3.180905 & 2.079846 & 1.449329 \\
\hline 35 & 3.338424 & 3.336617 & 0.879482 \\
\hline 36 & 2.501793 & 4.891682 & -0.338126 \\
\hline 37 & 4.25683 & 3.886631 & 1.057588 \\
\hline 38 & 0.15468 & 3.647197 & -0.916244 \\
\hline 39 & 0.270936 & 4.930895 & -1.502908 \\
\hline 40 & 0.379403 & 5.710188 & -0.74069 \\
\hline 41 & 1.117576 & 4.977569 & -2.196585 \\
\hline 42 & -0.655562 & 5.091718 & -2.052571 \\
\hline 43 & 3.964648 & 1.663558 & 2.069235 \\
\hline 44 & 1.738824 & 0.162093 & 1.759491 \\
\hline 45 & 2.825496 & -0.609557 & 2.239847 \\
\hline 46 & 3.240729 & -0.193542 & 3.164441 \\
\hline 47 & 2.418826 & -1.601486 & 2.43679 \\
\hline 48 & 3.614278 & -0.687417 & 1.483282 \\
\hline 49 & -7.6034 & -1.303006 & 0.096308 \\
\hline
\end{tabular}


Corresponding Z-matrix of compound $\mathbf{4 c}$

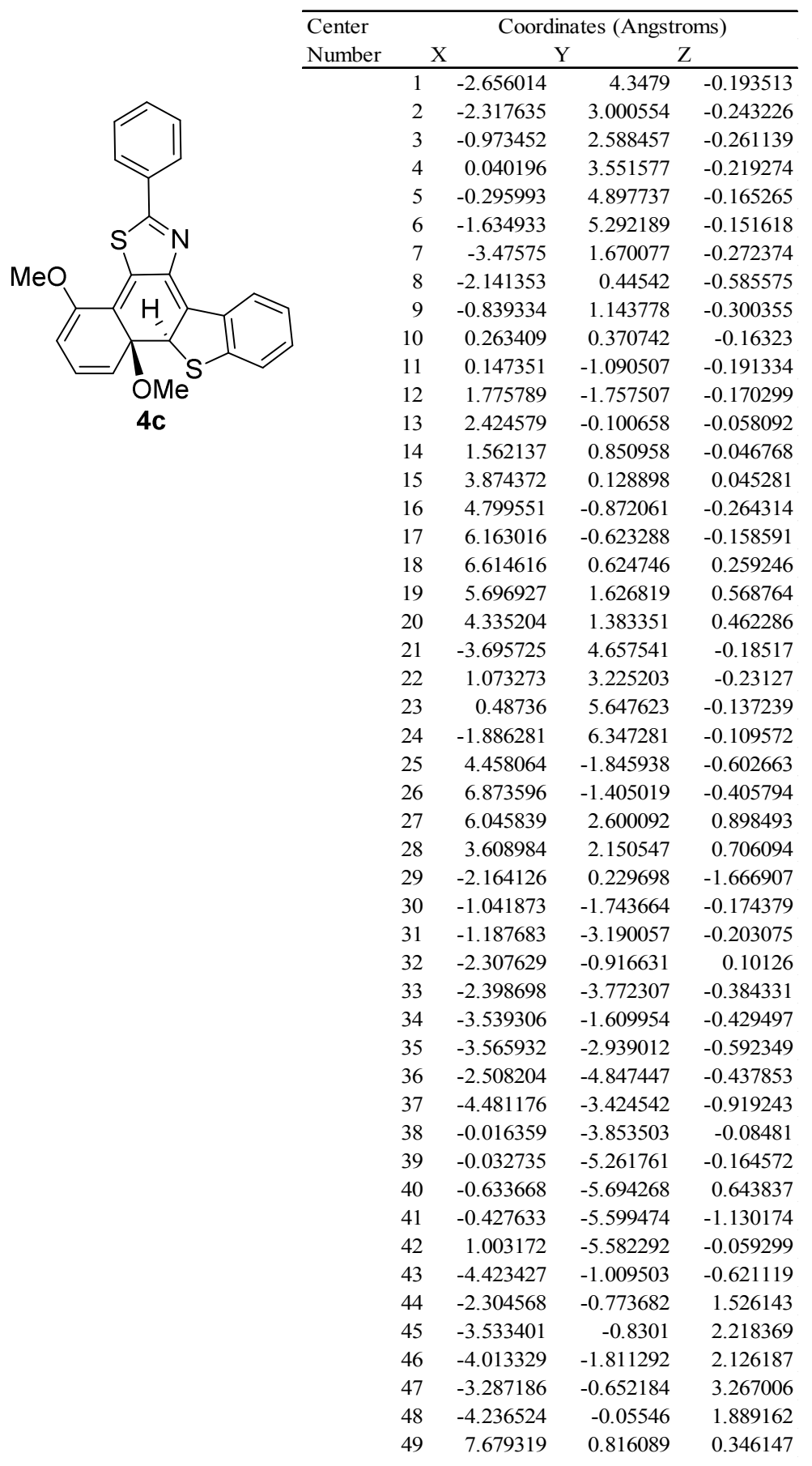


Corresponding Z-matrix of compound $4 \mathbf{s}$

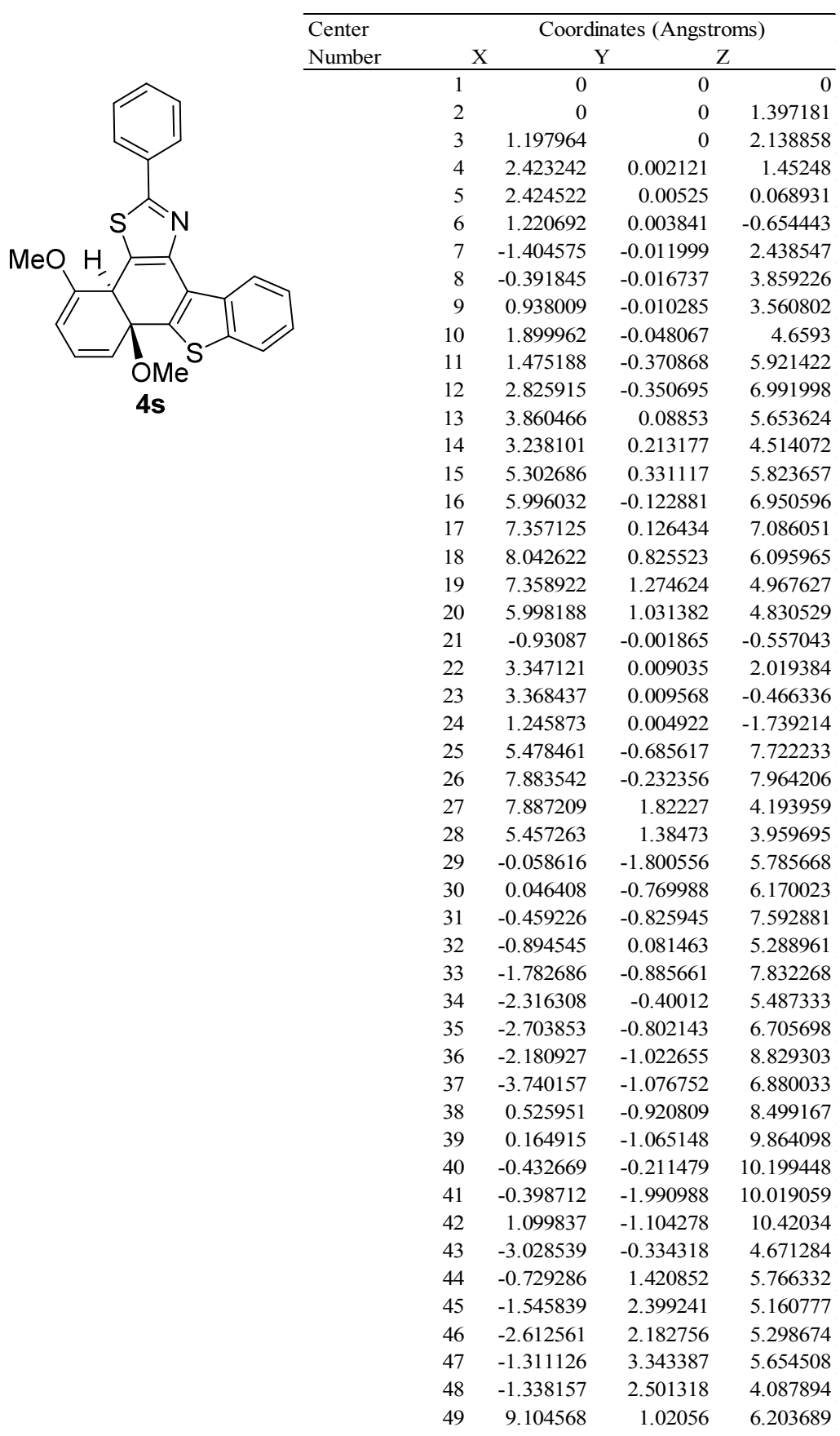


Corresponding Z-matrix of compound $\mathbf{4 a}$

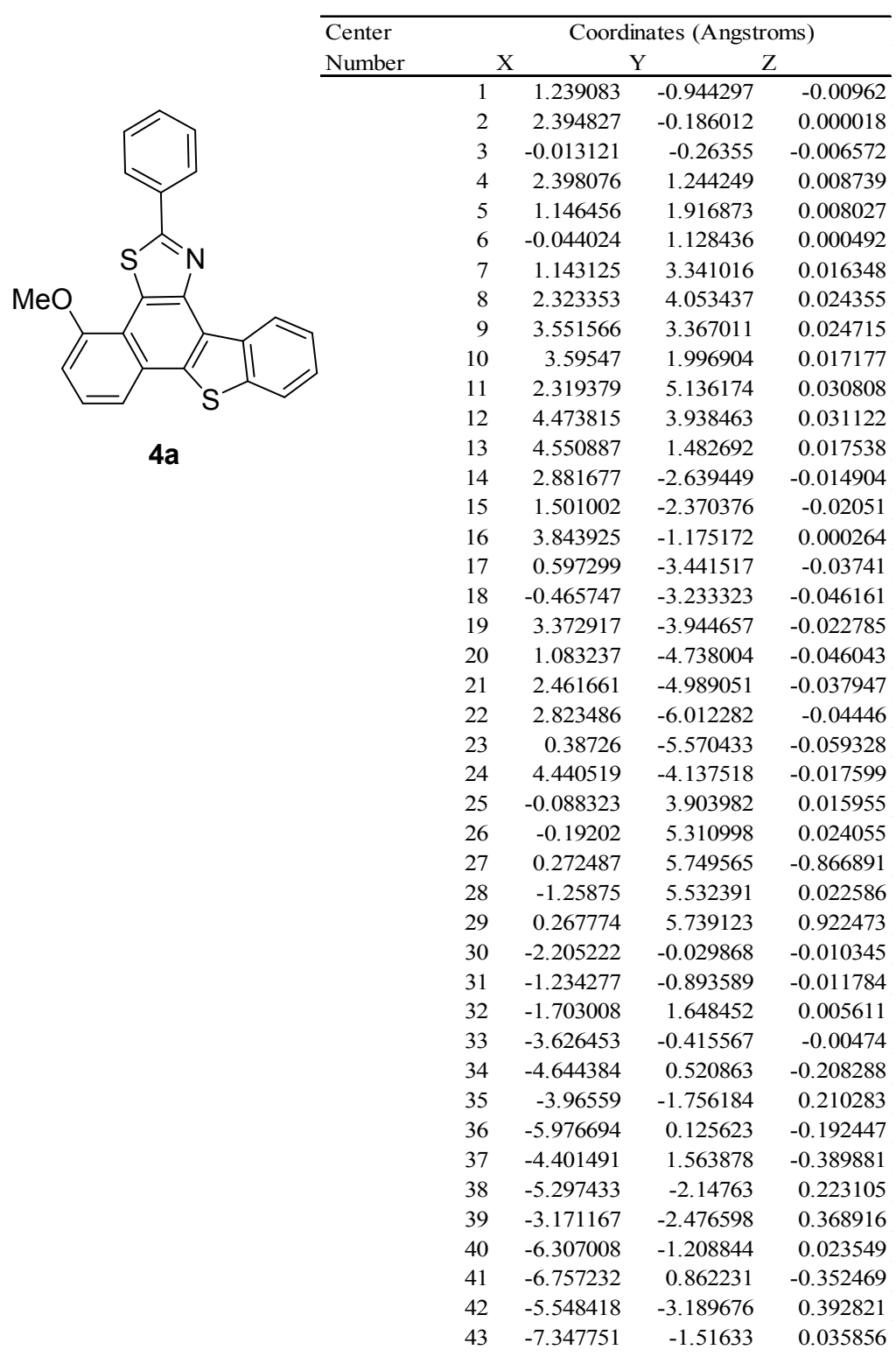




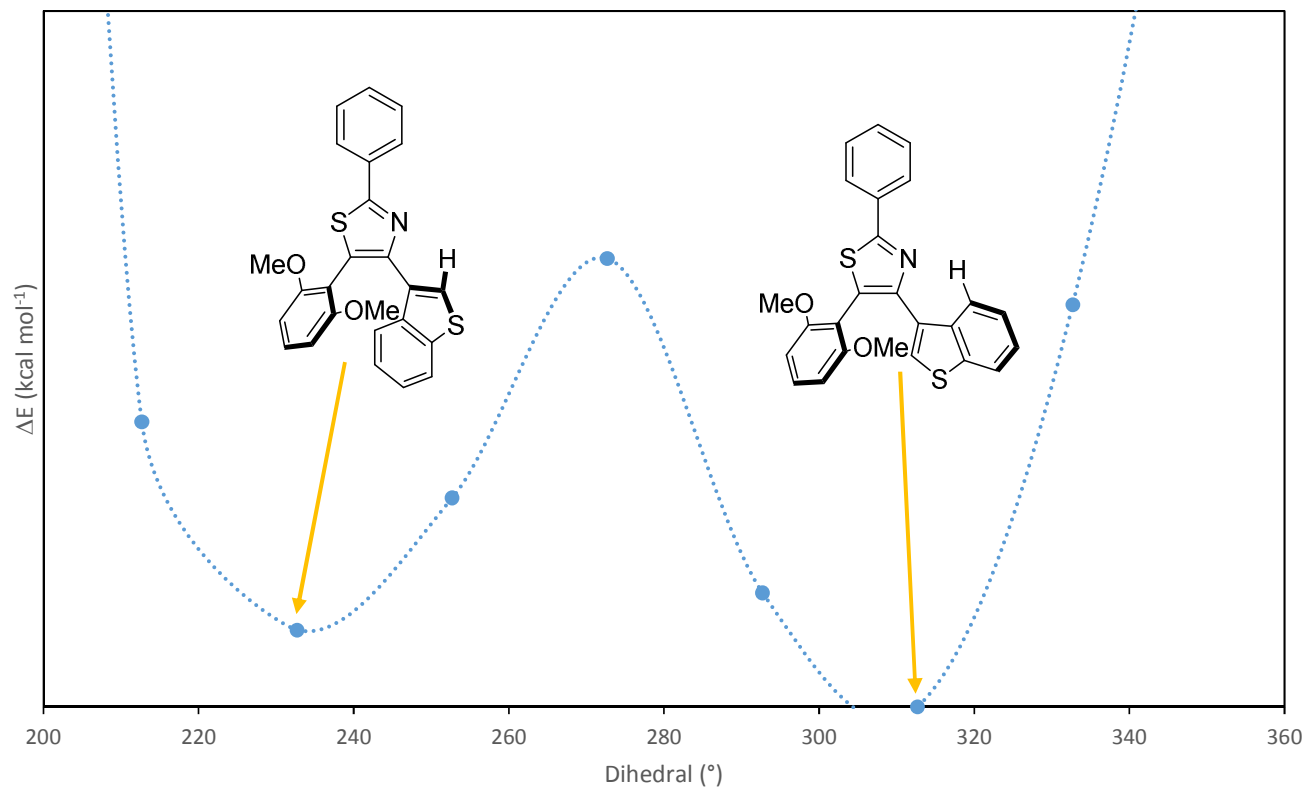

Figure S37 Ground state conformational PES (T=298.15 K) for compound 40

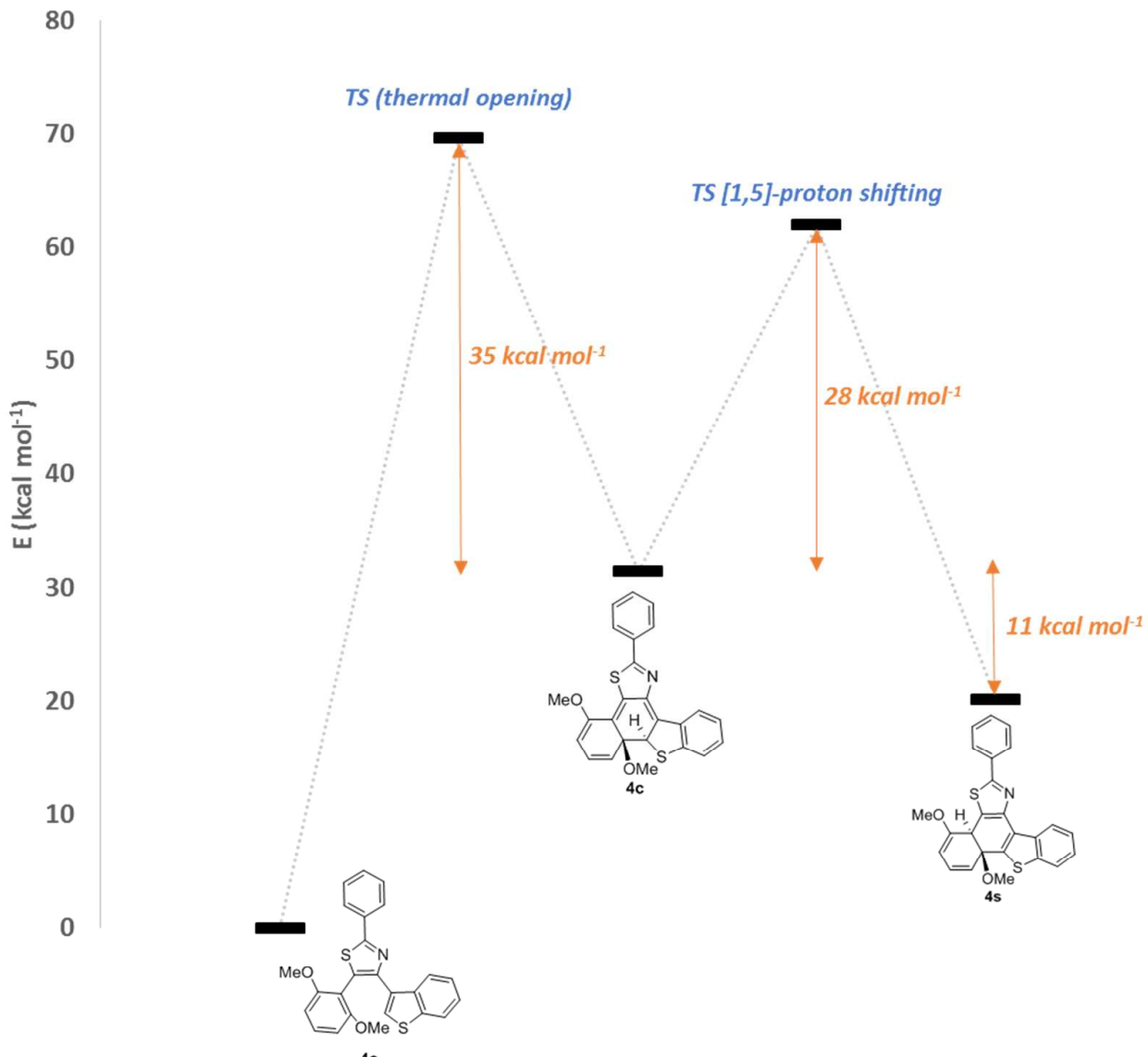

Figure S38 Ground state energetic profile for compound 4. TS stands for the respective Transient State calculated at the same level of theory 


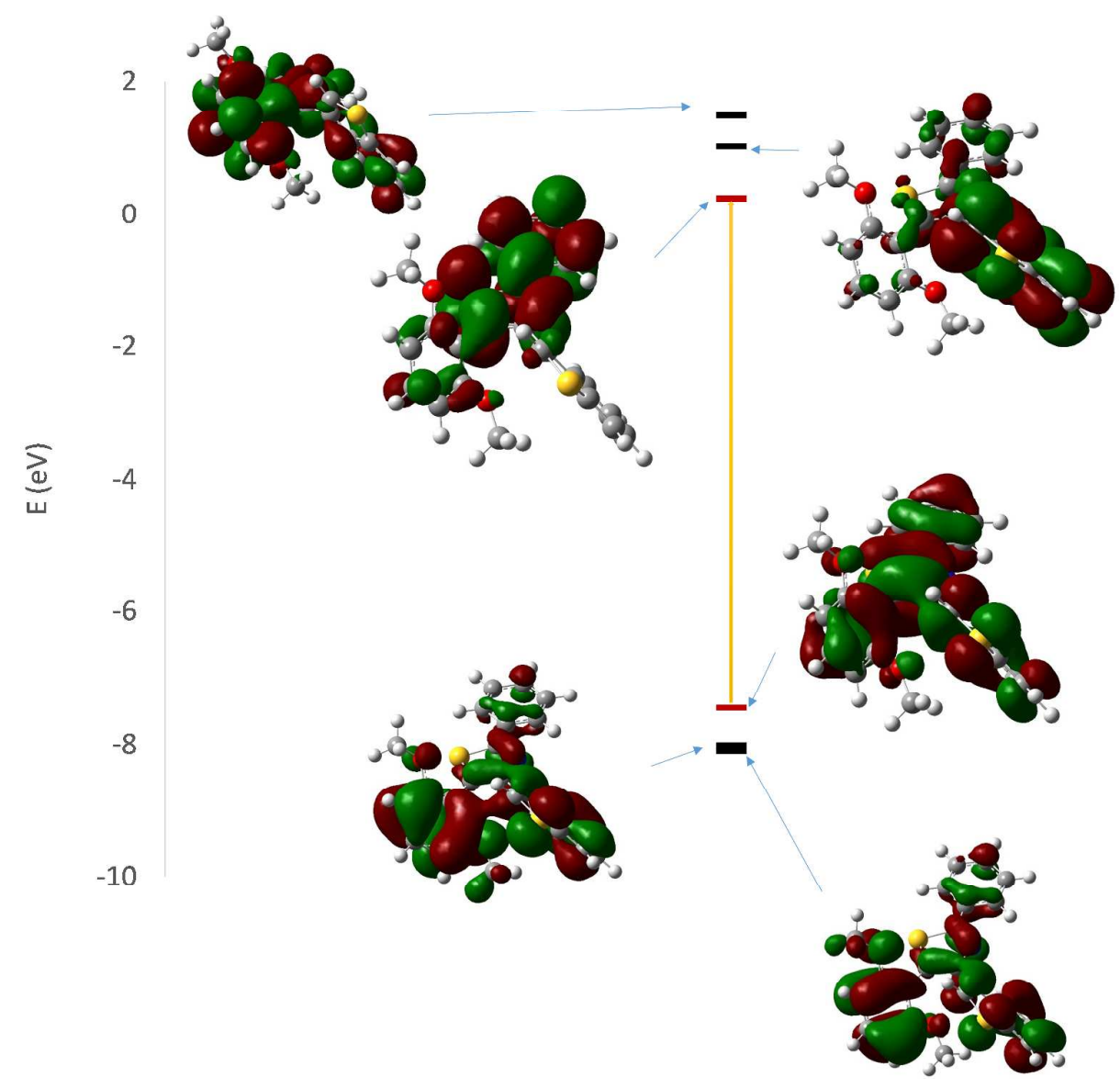

\begin{tabular}{|c|c|c|c|}
\hline Transition No. & Wavelength (nm) & Osc. Strength & Major contributions \\
\hline 1 & 300.238132073 & 0.6059 & HOMO->LUMO $(82 \%)$ \\
\hline 2 & 257.670546043 & 0.2979 & HOMO->LUMO+1 (62\%) \\
\hline 3 & 254.006958758 & 0.0554 & $\begin{array}{l}\text { HOMO-2->LUMO (19\%), HOMO-2->LUMO+2 (14\%), } \\
\text { HOMO-1->LUMO (22\%), HOMO-1->LUMO+2 (20\%) }\end{array}$ \\
\hline 4 & 247.718954325 & 0.0109 & HOMO-4->LUMO (21\%), HOMO-1->LUMO+1 (10\%) \\
\hline 5 & 242.320603224 & 0.055 & HOMO-5->LUMO $(20 \%)$ \\
\hline 6 & 242.088758229 & 0.0254 & HOMO-7->LUMO (10\%), HOMO-5->LUMO (31\%) \\
\hline 7 & 234.603649409 & 0.0692 & HOMO-7->LUMO (39\%), HOMO-6->LUMO (12\%) \\
\hline 8 & 234.111929303 & 0.096 & $\begin{array}{l}\text { HOMO-3->LUMO+1 (11\%), HOMO->LUMO+1 (10\%), } \\
\text { HOMO->LUMO+2 (24\%) }\end{array}$ \\
\hline 9 & 225.186778742 & 0.0651 & $\begin{array}{l}\text { HOMO-7->LUMO (11\%), HOMO-4->LUMO ( } 16 \%) \\
\text { HOMO-3->LUMO (17\%), HOMO->LUMO+2 }(20 \%)\end{array}$ \\
\hline 10 & 220.395230005 & 0.0431 & HOMO-1->LUMO+1 (23\%), HOMO->LUMO+2 (11\%) \\
\hline
\end{tabular}

Figure S39 MO energy diagram of compound 40 with the nature of the corresponding transitions. The yellow arrow symbolizes the "photochromic transition" 


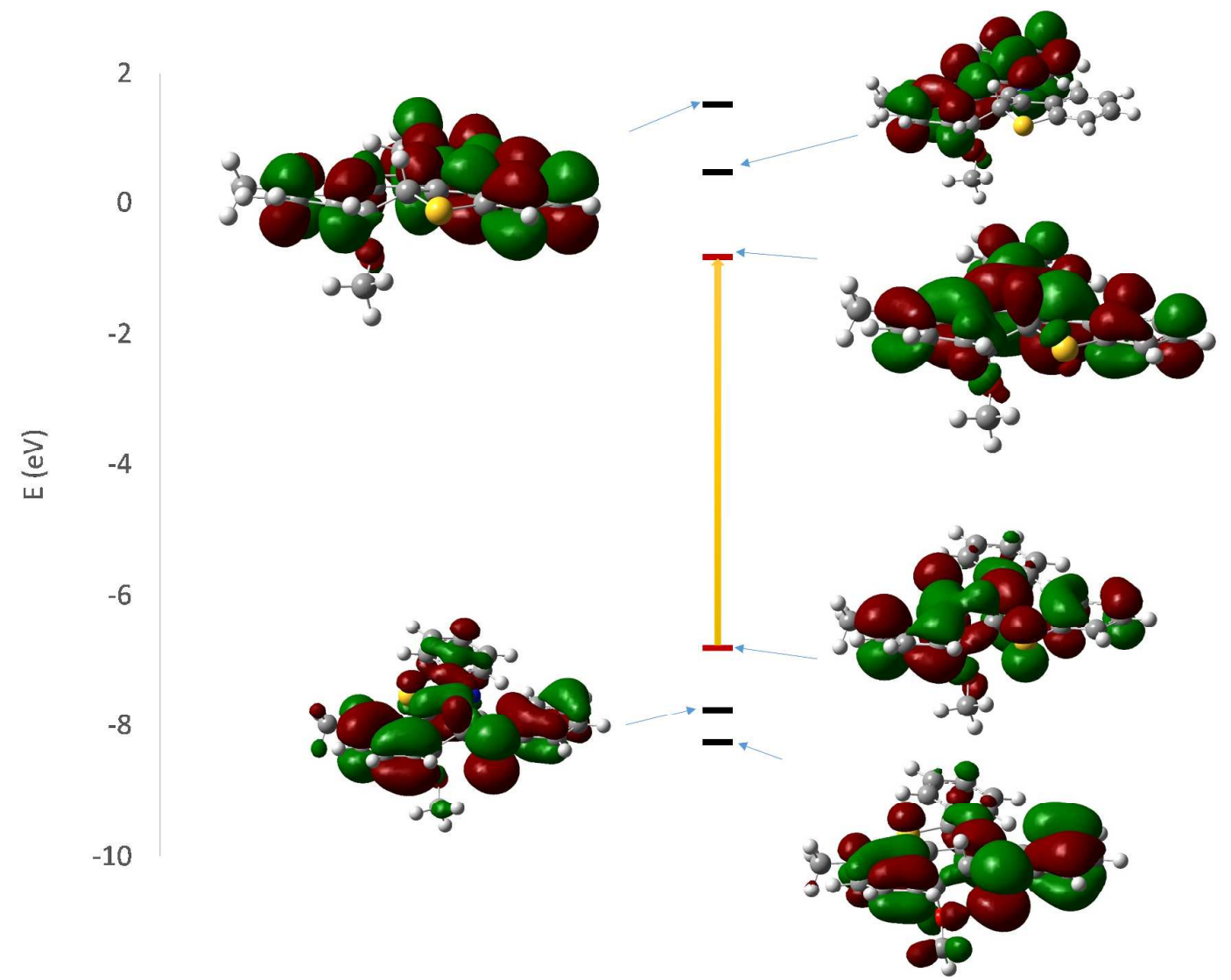

\begin{tabular}{|c|c|c|c|}
\hline Transition No. & Wavelength (nm) & Osc. Strength & Major contributions \\
\hline 1 & 479.6631718 & 0.2793 & HOMO->LUMO (94\%) \\
\hline 2 & 346.3028229 & 0.3935 & HOMO-1->LUMO (89\%) \\
\hline 3 & 314.8863124 & 0.2127 & HOMO-2->LUMO (40\%), HOMO->LUMO+1 (48\%) \\
\hline 4 & 302.900754 & 0.17 & HOMO-2->LUMO (47\%), HOMO->LUMO+1 (34\%) \\
\hline 5 & 275.62265 & 0.2032 & HOMO-5->LUMO (33\%), HOMO-3->LUMO (45\%) \\
\hline 6 & 266.9925633 & 0.1991 & $\begin{array}{l}\text { HOMO-7->LUMO }(30 \%) \text {, HOMO-5->LUMO }(26 \%) \text {, } \\
\text { HOMO-3->LUMO }(21 \%)\end{array}$ \\
\hline 7 & 261.7504521 & 0.0678 & HOMO-7->LUMO (48\%), HOMO-5->LUMO (19\%) \\
\hline 8 & 251.7274819 & 0.0202 & $\begin{array}{l}\text { HOMO-1->LUMO+5 (15\%), HOMO->LUMO+2 (18\%), } \\
\text { HOMO->LUMO+5 (36\%) }\end{array}$ \\
\hline 9 & 250.6283463 & 0.0455 & $\begin{array}{l}\text { HOMO-6->LUMO (14\%), HOMO-4->LUMO (11\%), } \\
\text { HOMO-1->LUMO+1 (15\%), HOMO->LUMO+4 (28\%) }\end{array}$ \\
\hline 10 & 247.7437039 & 0.1459 & $\begin{array}{l}\text { HOMO->LUMO+2 (31\%), HOMO->LUMO+3 (13\%), } \\
\text { HOMO->LUMO+5 (11\%) }\end{array}$ \\
\hline
\end{tabular}

Figure $540 \mathrm{MO}$ energy diagram of compound $4 \mathrm{c}$ with the nature of the corresponding transitions. The yellow arrow symbolizes the "photochromic transition" 


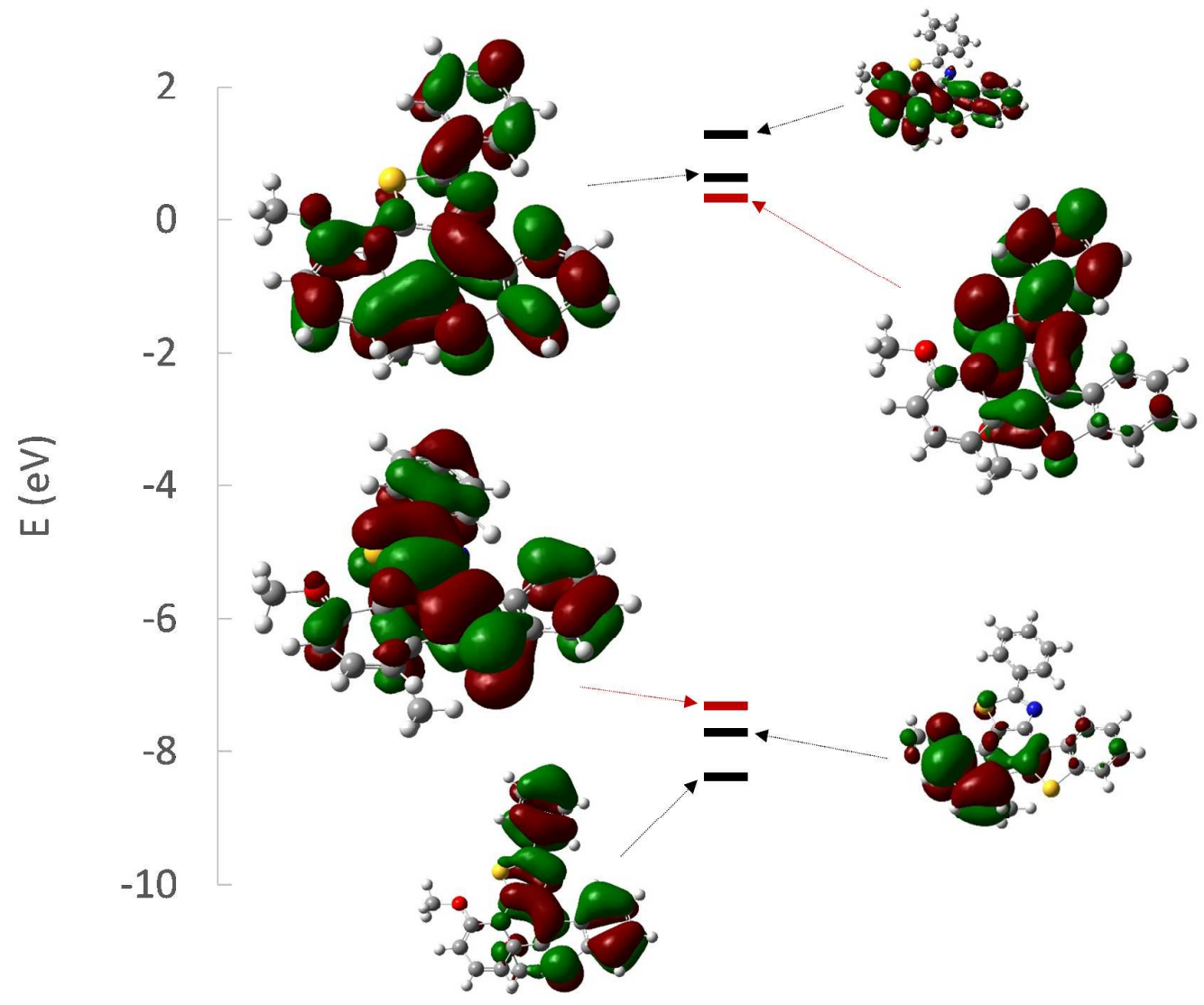

\begin{tabular}{|c|c|c|c|}
\hline Transition No. & Wavelength (nm) & Osc. Strength & Major contributions \\
\hline 1 & 300.4855351 & 0.0545 & HOMO->LUMO (79\%) \\
\hline 2 & 281.9148608 & 0.703 & HOMO->LUMO+1 $(76 \%)$ \\
\hline 3 & 275.5246486 & 0.1184 & $\begin{array}{l}\text { HOMO-1->LUMO (19\%), HOMO-1->LUMO+1 (43\%), } \\
\text { HOMO-1->LUMO+2 (25\%) }\end{array}$ \\
\hline 4 & 251.0495619 & 0.0074 & $\begin{array}{l}\text { HOMO-3->LUMO+1 (28\%), HOMO->LUMO+2 (16\%), } \\
\text { HOMO->LUMO+4 (11\%) }\end{array}$ \\
\hline 5 & 245.448373 & 0.0687 & HOMO-1->LUMO (44\%), HOMO-1->LUMO+2 (24\%) \\
\hline 6 & 241.0720137 & 0.0103 & $\begin{array}{l}\text { HOMO-4->LUMO (39\%), HOMO-4->LUMO+1 (11\%), } \\
\text { HOMO-2->LUMO+3 (14\%), HOMO->LUMO+3 (13\%) }\end{array}$ \\
\hline 7 & 233.7984851 & 0.1177 & HOMO-2->LUMO (25\%), HOMO->LUMO+2 (15\%) \\
\hline 8 & 231.9700207 & 0.019 & HOMO-7->LUMO (69\%) \\
\hline 9 & 230.0631583 & 0.0839 & HOMO-2->LUMO (28\%), HOMO->LUMO+2 (15\%) \\
\hline 10 & 226.9634734 & 0.2399 & $\begin{array}{l}\text { HOMO-2->LUMO+1 (22\%), HOMO->LUMO+2 (24\%), } \\
\text { HOMO->LUMO+5 (13\%) }\end{array}$ \\
\hline
\end{tabular}

Figure S41 MO energy diagram of compound $4 s$ with the nature of the corresponding transitions 


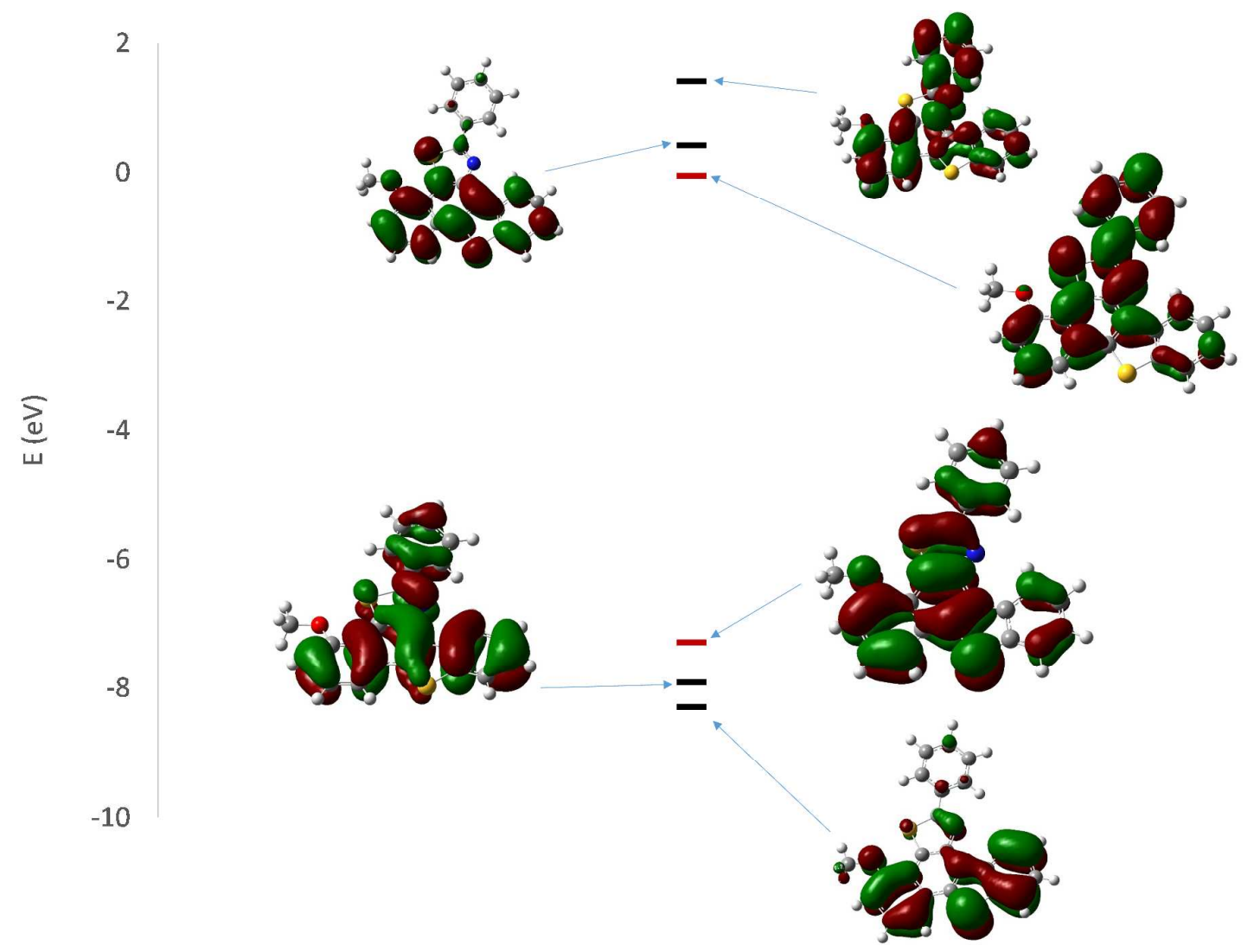

\begin{tabular}{|c|c|c|c|}
\hline Transition No. & Wavelength (nm) & Osc. Strength & Major contributions \\
\hline 1 & 321.058955 & 0.5244 & HOMO->LUMO $(83 \%)$ \\
\hline 2 & 298.3955154 & 0.0023 & HOMO-1->LUMO (21\%), HOMO->LUMO+1 (69\%) \\
\hline 3 & 266.1557578 & 0.6924 & HOMO-1->LUMO (53\%), HOMO->LUMO+1 (16\%) \\
\hline 4 & 264.3229792 & 0.7177 & HOMO-1->LUMO+1 (66\%) \\
\hline 5 & 251.5232115 & 0.1721 & HOMO-2->LUMO (30\%), HOMO-2->LUMO+1 (29\%) \\
\hline 6 & 243.9512359 & 0.1264 & HOMO-4->LUMO (41\%), HOMO->LUMO+2 (12\%) \\
\hline 7 & 240.5575022 & 0.1557 & $\begin{array}{l}\text { HOMO-3->LUMO }(15 \%), \text { HOMO-2->LUMO }(10 \%) \\
\text { HOMO->LUMO+2 }(16 \%)\end{array}$ \\
\hline 8 & 238.5212325 & 0.0002 & HOMO-7->LUMO (83\%) \\
\hline 9 & 227.0757081 & 0.076 & $\begin{array}{l}\text { HOMO-2->LUMO }(17 \%), \text { HOMO-2->LUMO+1 ( } 35 \%) \text {, } \\
\text { HOMO->LUMO+2 (17\%) }\end{array}$ \\
\hline 10 & 222.053079 & 0.0855 & $\begin{array}{l}\text { HOMO-2->LUMO+4 (10\%), HOMO-1->LUMO+4 } \\
(12 \%), \text { HOMO->LUMO+4 (30\%) }\end{array}$ \\
\hline
\end{tabular}




\section{Details on compound 6}

Corresponding Z-matrix of compound 6o_OAc

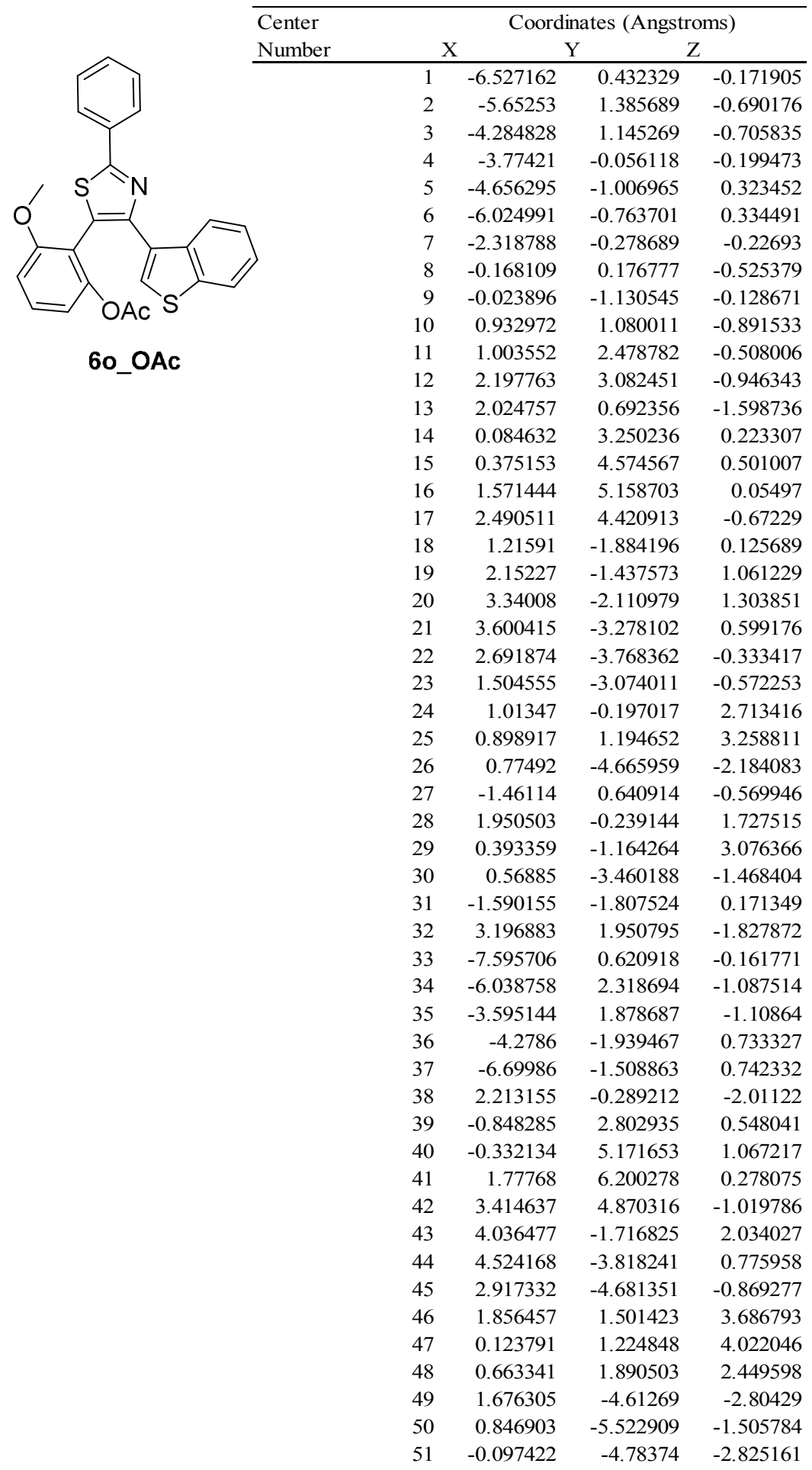


Corresponding Z-matrix of compound $\mathbf{6 c \_ O A c}$

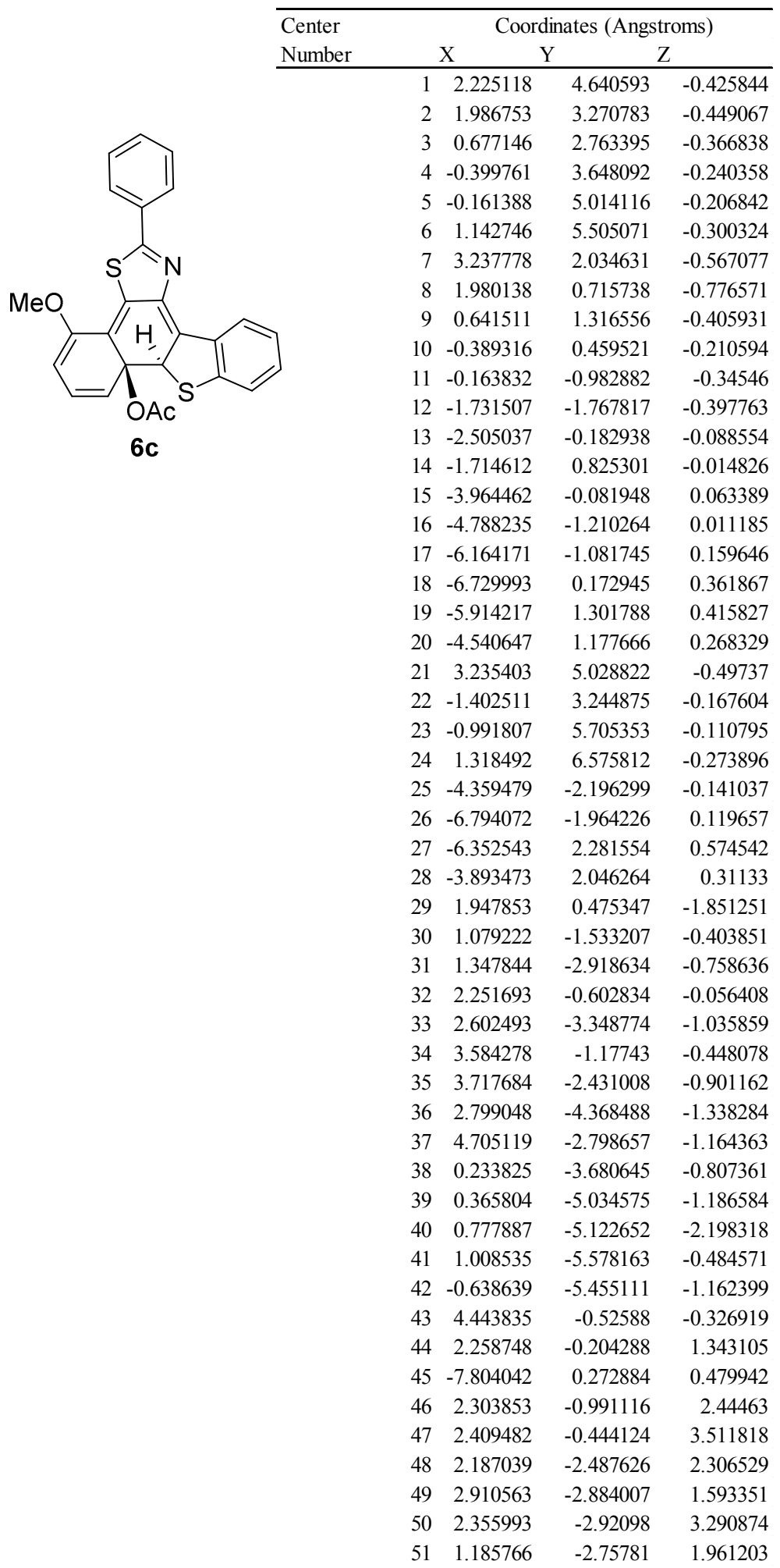


Corresponding Z-matrix of compound 6s_OAc

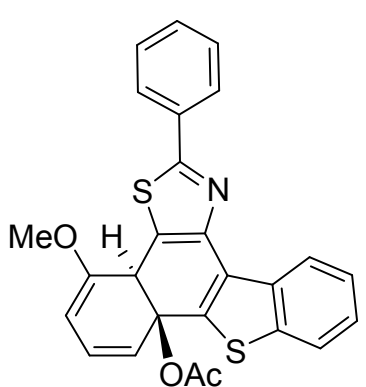

6s

\begin{tabular}{|c|c|c|c|}
\hline \multirow{2}{*}{$\begin{array}{l}\text { Center } \\
\text { Number }\end{array}$} & \multicolumn{3}{|c|}{ Coordinates (Angstroms) } \\
\hline & $X$ & Y & $\mathrm{Z}$ \\
\hline 1 & -2.294925 & 4.573582 & -0.61811 \\
\hline 2 & -2.023658 & 3.209547 & -0.485312 \\
\hline 3 & -0.706338 & 2.721277 & -0.388581 \\
\hline 4 & 0.362789 & 3.63145 & -0.423825 \\
\hline 5 & 0.095805 & 4.98238 & -0.552145 \\
\hline 6 & -1.22448 & 5.451625 & -0.649376 \\
\hline 7 & -3.19868 & 1.916825 & -0.435061 \\
\hline 8 & -1.930039 & 0.727157 & -0.268548 \\
\hline 9 & -0.684377 & 1.280854 & -0.274777 \\
\hline 10 & 0.47753 & 0.398201 & -0.241856 \\
\hline 11 & 0.318988 & -0.93569 & -0.508619 \\
\hline 12 & 1.857383 & -1.709292 & -0.441833 \\
\hline 13 & 2.596575 & -0.170576 & -0.067546 \\
\hline 14 & 1.755683 & 0.8235 & 0.008875 \\
\hline 15 & 4.043446 & -0.029805 & 0.165645 \\
\hline 16 & 4.953685 & -1.005379 & -0.25462 \\
\hline 17 & 6.315196 & -0.839829 & -0.026515 \\
\hline 18 & 6.782719 & 0.3029 & 0.617207 \\
\hline 19 & 5.880972 & 1.279818 & 1.035417 \\
\hline 20 & 4.519669 & 1.116257 & 0.813708 \\
\hline 21 & -3.314288 & 4.93591 & -0.695145 \\
\hline 22 & 1.377106 & 3.258549 & -0.341641 \\
\hline 23 & 0.916097 & 5.692066 & -0.577144 \\
\hline 24 & -1.410038 & 6.515686 & -0.751561 \\
\hline 25 & 4.604677 & -1.892676 & -0.775049 \\
\hline 26 & 7.012351 & -1.601483 & -0.359497 \\
\hline 27 & 6.240053 & 2.171201 & 1.539308 \\
\hline 28 & 3.810202 & 1.867626 & 1.142018 \\
\hline 29 & -1.026636 & -1.487201 & -0.895053 \\
\hline 30 & -1.232638 & -2.984948 & -0.854302 \\
\hline 31 & -2.140665 & -0.761849 & -0.105449 \\
\hline 32 & -2.47914 & -3.494437 & -0.883531 \\
\hline 33 & -3.479911 & -1.278244 & -0.565867 \\
\hline 34 & -3.605825 & -2.574549 & -0.884759 \\
\hline 35 & -2.663744 & -4.558372 & -0.950419 \\
\hline 36 & -4.580054 & -2.967164 & -1.159963 \\
\hline 37 & -0.085458 & -3.673496 & -0.901295 \\
\hline 38 & -0.159474 & -5.091425 & -0.948801 \\
\hline 39 & -0.675179 & -5.479605 & -0.0652 \\
\hline 40 & -0.67979 & -5.419652 & -1.854256 \\
\hline 41 & 0.869233 & -5.446512 & -0.963198 \\
\hline 42 & -4.33395 & -0.611121 & -0.583287 \\
\hline 43 & -1.831475 & -1.147053 & 1.278361 \\
\hline 44 & 7.845697 & 0.432985 & 0.791735 \\
\hline 45 & -1.194697 & -1.199437 & -1.948261 \\
\hline 46 & -2.598182 & -1.002506 & 2.375429 \\
\hline 47 & -2.135195 & -1.394548 & 3.422565 \\
\hline 48 & -3.974378 & -0.399196 & 2.278765 \\
\hline 49 & -3.977406 & 0.531359 & 1.709693 \\
\hline 50 & -4.65286 & -1.101619 & 1.790032 \\
\hline 51 & -4.325661 & -0.208504 & 3.291513 \\
\hline
\end{tabular}


Corresponding Z-matrix of compound 6o_OMe<smiles>COc1cccc(OC(C)=O)c1-c1sc(-c2ccccc2)nc1-c1csc2ccccc12</smiles>

\begin{tabular}{lrrrr}
\hline Center & \multicolumn{4}{c}{ Coordinates (Angstroms) } \\
Number & \multicolumn{3}{c}{$\mathrm{X}$} & \multicolumn{2}{c}{$\mathrm{Z}$} \\
\hline & 1 & -3.287033 & -3.896035 & 0.651871 \\
& 2 & -2.69677 & -2.654545 & 0.901691 \\
& 3 & -1.446535 & -2.30551 & 0.358522 \\
& 4 & -0.782142 & -3.226514 & -0.466165 \\
& 5 & -1.366394 & -4.455055 & -0.719595 \\
& 6 & -2.611118 & -4.790045 & -0.161912 \\
& 7 & -3.363498 & -1.346477 & 1.851433 \\
& 8 & -1.98105 & -0.352609 & 1.504244 \\
9 & -1.05407 & -0.95642 & 0.721133 \\
& 10 & 0.174577 & -0.315785 & 0.221288 \\
11 & 0.238697 & 0.90574 & -0.406898 \\
12 & 1.886181 & 1.231268 & -0.840584 \\
13 & 2.358317 & -0.303826 & -0.167435 \\
14 & 1.368313 & -0.982338 & 0.342159 \\
15 & 3.752318 & -0.777825 & -0.160669 \\
16 & 4.785873 & -0.048199 & -0.756793 \\
17 & 6.08938 & -0.531227 & -0.733862 \\
18 & 6.374413 & -1.746995 & -0.117647 \\
19 & 5.348417 & -2.478523 & 0.477701 \\
20 & 4.045306 & -1.999245 & 0.458174 \\
21 & -4.250702 & -4.150618 & 1.079924 \\
22 & 0.182514 & -2.969169 & -0.889498 \\
23 & -0.856454 & -5.170234 & -1.356633 \\
24 & -3.051056 & -5.759606 & -0.370717 \\
25 & 4.581345 & 0.901055 & -1.243871 \\
26 & 6.882995 & 0.043345 & -1.199655 \\
27 & 5.564023 & -3.426103 & 0.960338 \\
28 & 3.239799 & -2.559258 & 0.919644 \\
29 & -1.948016 & 0.651289 & 1.902268 \\
30 & -0.87454 & 1.809122 & -0.740084 \\
31 & -2.024235 & 1.310304 & -1.395986 \\
32 & -0.864769 & 3.163729 & -0.397717 \\
33 & -3.131524 & 2.132589 & -1.605488 \\
& 49 & -0.317406 & 2.966127 & 2.364147 \\
& 50 & -0.080234 & 3.234552 & 3.391594 \\
51 & -1.370599 & 3.162847 & 2.154296
\end{tabular}


Corresponding Z-matrix of compound 6a

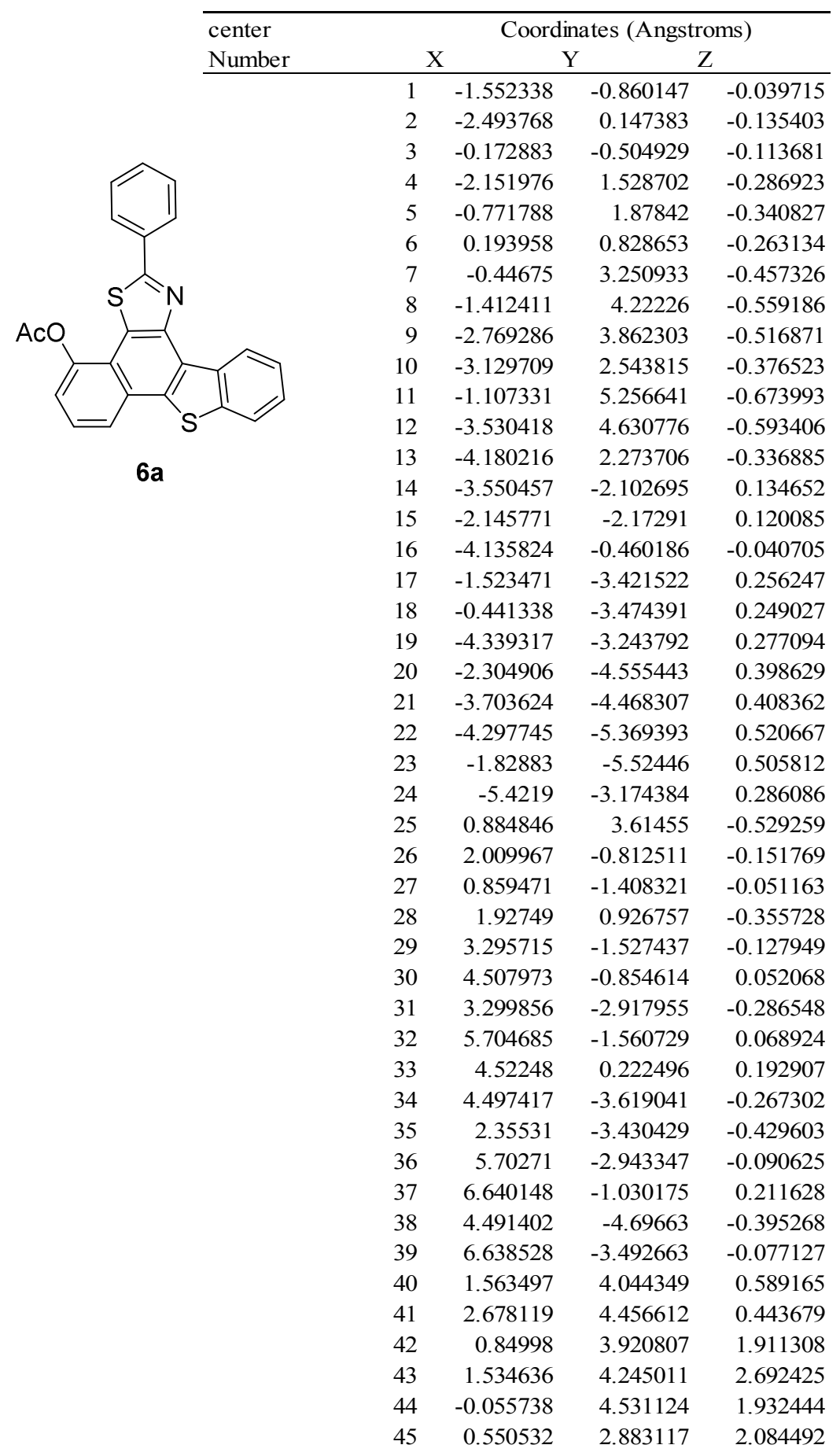


Secondary anti-parallel conformer of compound $\mathbf{6 0}$ :
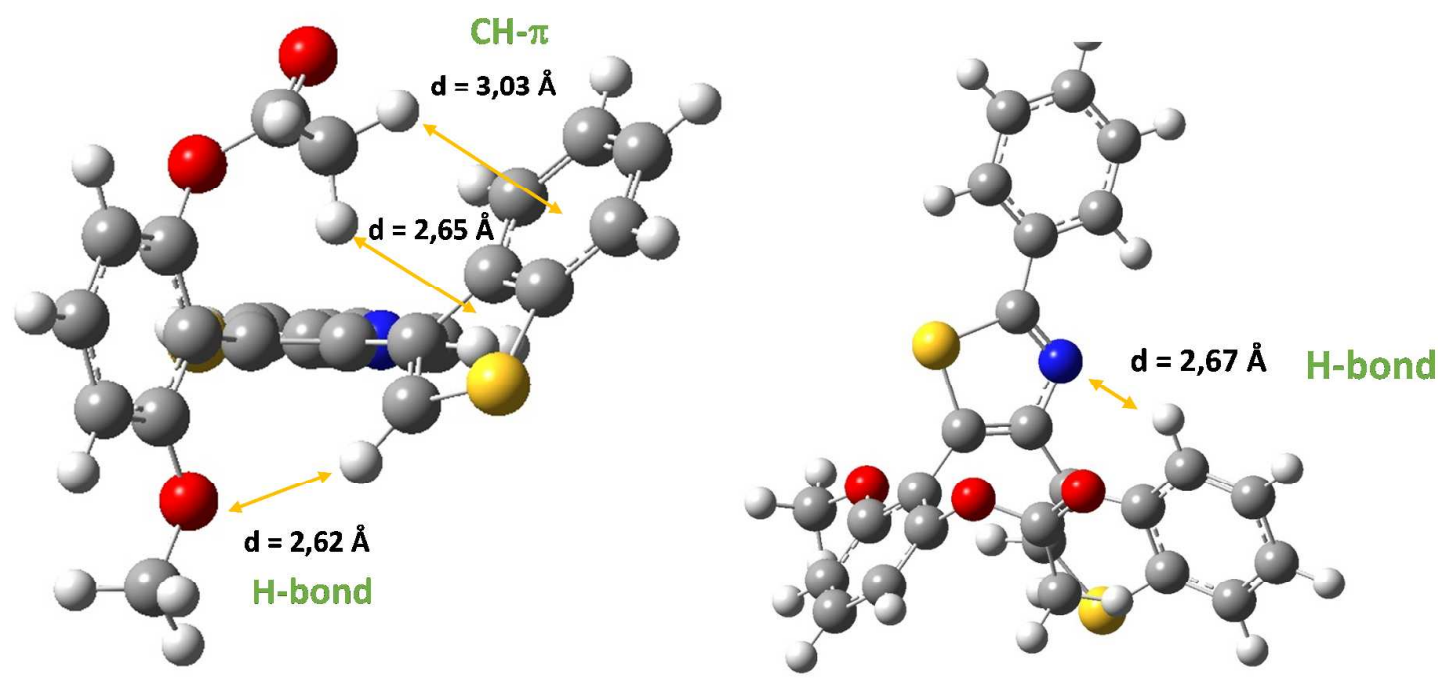

Figure $S 43$ Conformation of compound 6 responsible for the AcOH release, with the respective relevant distances and interactions 


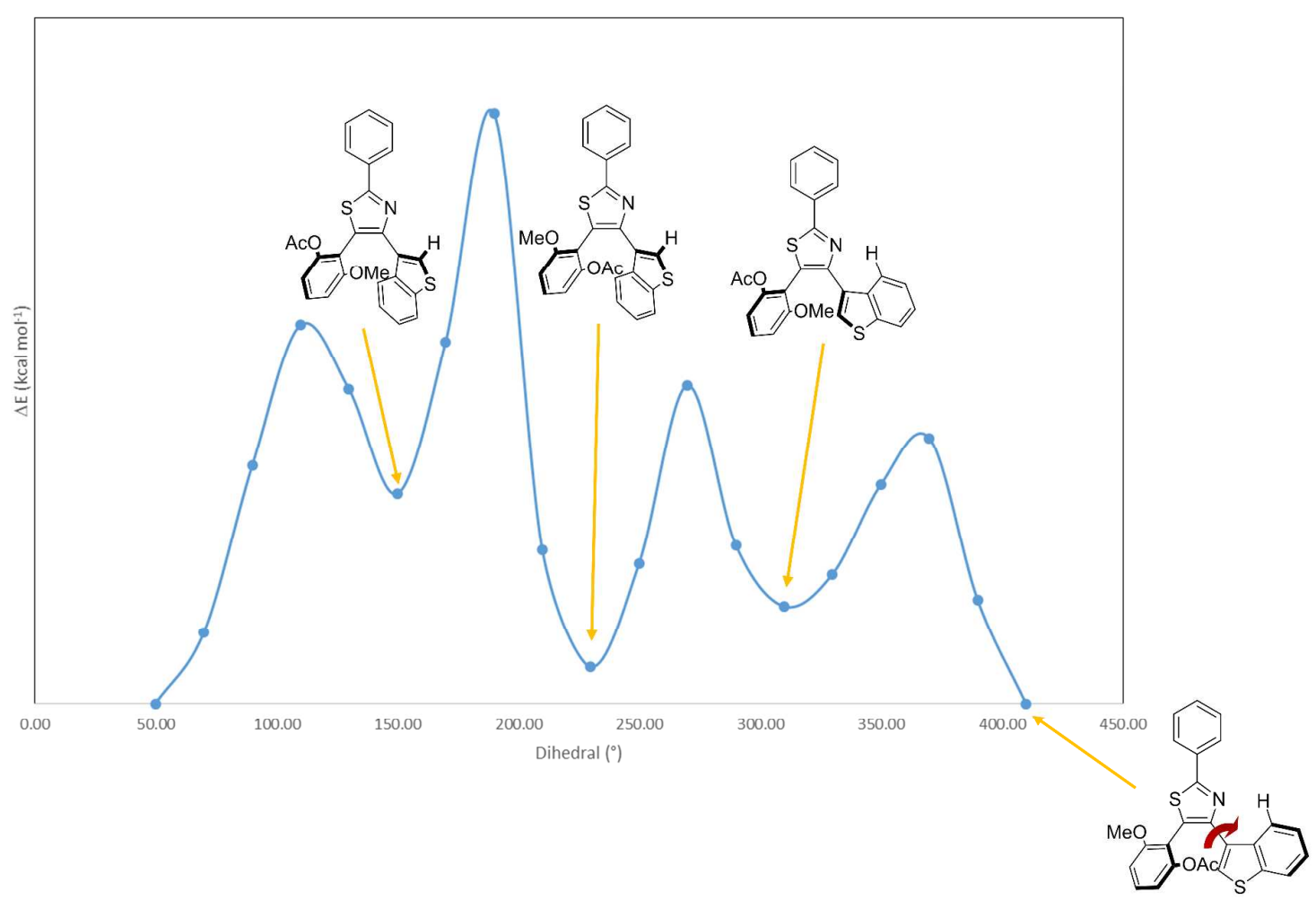

Figure S 44 Ground state conformational equilibrium $(T=298.15 K)$ for compound 60 in vacuum 


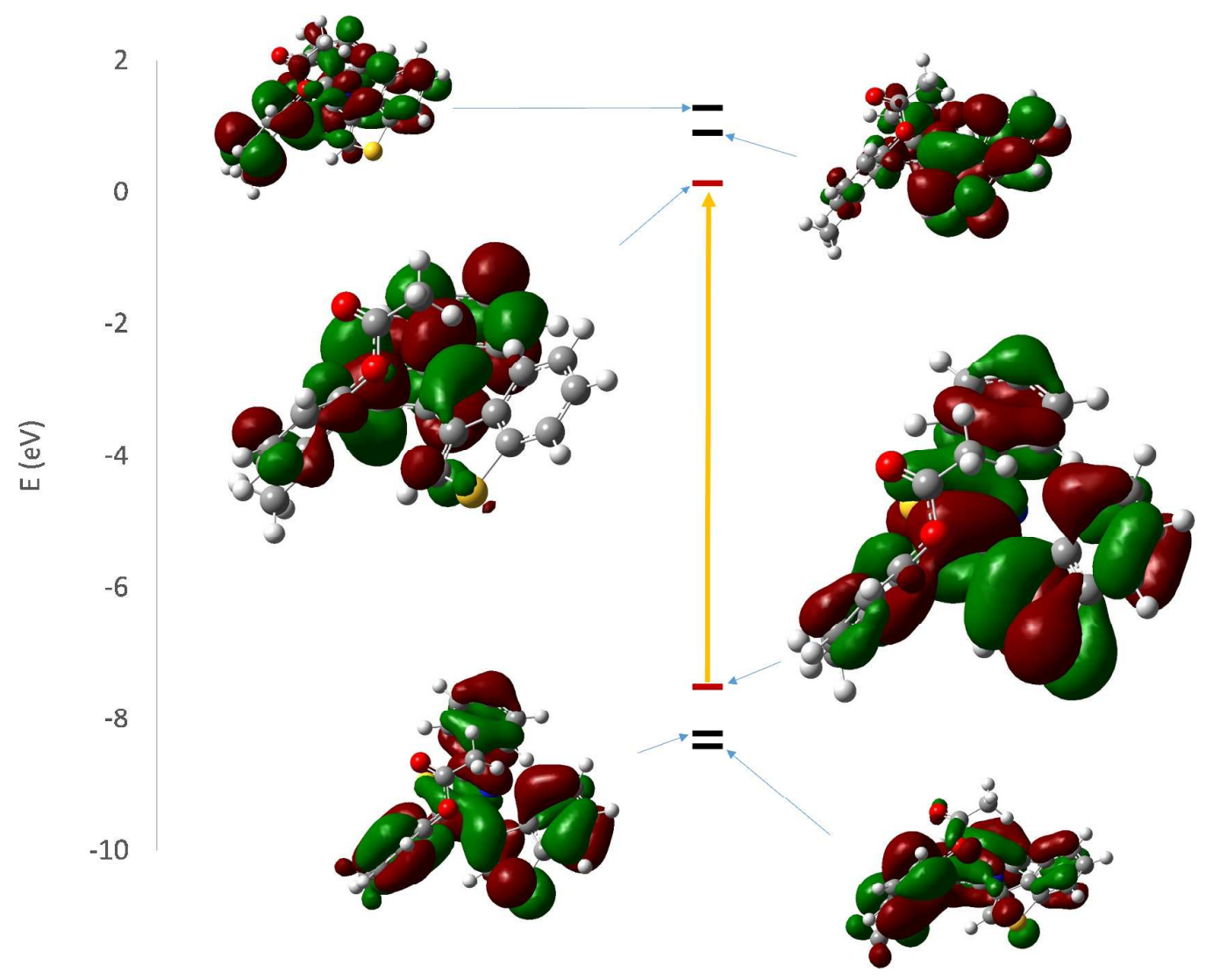

\begin{tabular}{llll}
\hline Transition No. & Wavelength $(\mathrm{nm})$ & Osc. Strength & Major contributions \\
\hline 1 & 297.9580799 & 0.4754 & HOMO-1->LUMO (10\%), HOMO->LUMO (80\%) \\
2 & 261.5130492 & 0.4457 & HOMO->LUMO+1 (67\%) \\
3 & 252.0550055 & 0.0787 & $\begin{array}{l}\text { HOMO-2->LUMO (21\%), HOMO-2->LUMO+2 (12\%), } \\
\text { HOMO-1->LUMO (23\%) }\end{array}$ \\
4 & 244.2973274 & 0.0841 & $\begin{array}{l}\text { HOMO-3->LUMO+1 (18\%), HOMO-1->LUMO (11\%) } \\
\text { HOMO-5->LUMO (30\%), HOMO-4->LUMO (11\%) }\end{array}$ \\
5 & 243.0569234 & 0.007 & $\begin{array}{l}\text { HOMO-7->LUMO (35\%), HOMO-5->LUMO (19\%) } \\
6\end{array}$ \\
239.9569116 & 0.0116 & $\begin{array}{l}\text { HOMO-3->LUMO+1 (11\%), HOMO-1->LUMO (13\%), } \\
\text { HOMO->LUMO+2 (26\%) }\end{array}$ \\
8 & 235.9744516 & 0.0924 & HOMO-7->LUMO (15\%), HOMO-1->LUMO (10\%), \\
& 233.6222661 & 0.0785 & $\begin{array}{l}\text { HOMO->LUMO+2 (17\%) } \\
\text { HOMO-7->LUMO (12\%), HOMO-2->LUMO (16\%), }\end{array}$ \\
10 & 226.0283606 & 0.0231 & $\begin{array}{l}\text { HOMO->LUMO+2 (14\%) } \\
\text { HOMO->LUMO+6 (21\%), HOMO->LUMO+9 (10\%) }\end{array}$
\end{tabular}

Figure S45 MO energy diagram of compound 6o_OAc with the nature of the corresponding transitions 


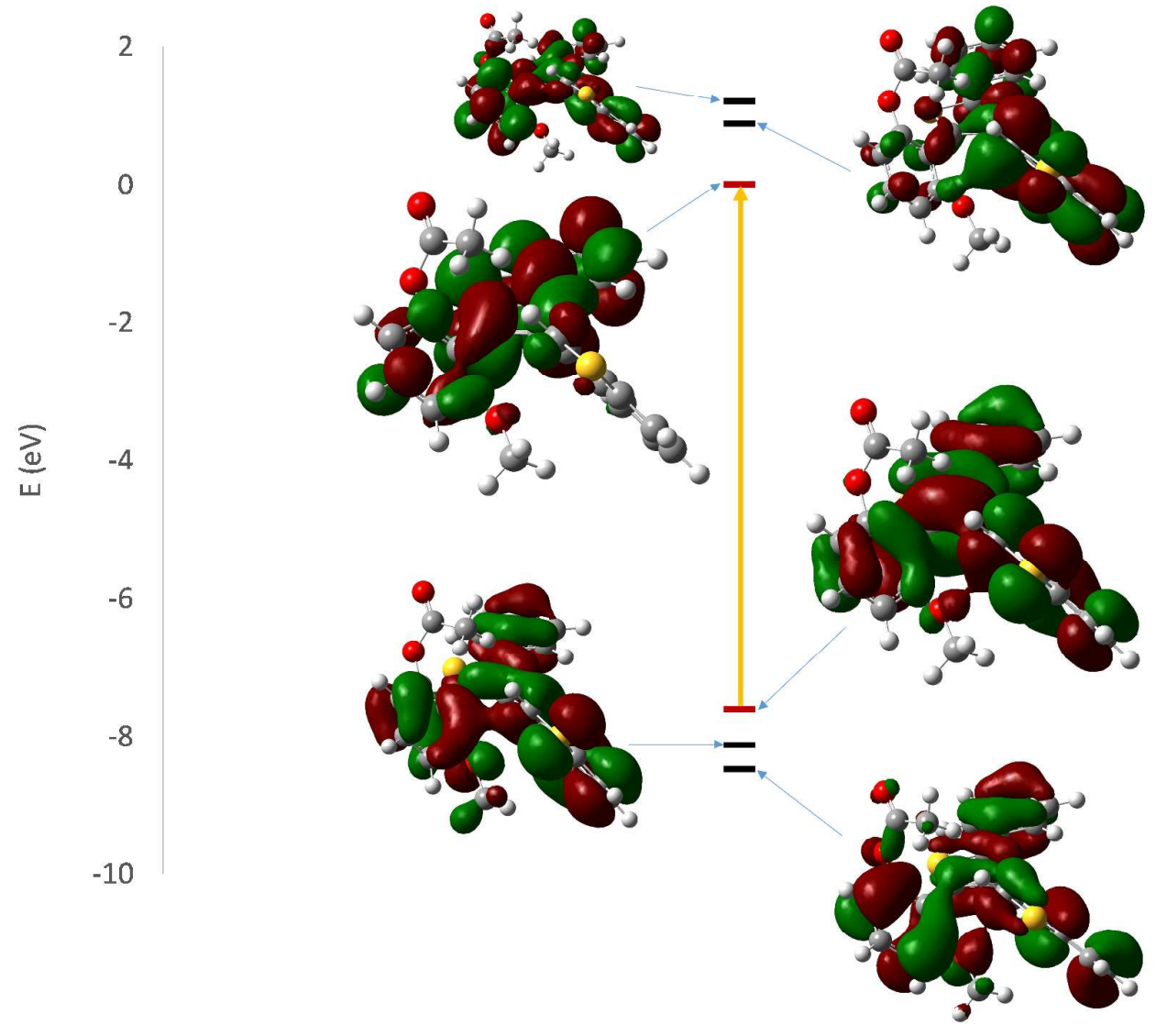

\begin{tabular}{llll}
\hline Transition No. & Wavelength $(\mathrm{nm})$ & Osc. Strength & Major contributions \\
\hline 1 & 303.9850356 & 0.5754 & HOMO-1->LUMO (12\%), HOMO->LUMO (80\%) \\
2 & 259.1787458 & 0.4242 & HOMO-1->LUMO (11\%), HOMO->LUMO+1 (57\%) \\
3 & 257.6330659 & 0.0346 & HOMO-2->LUMO (19\%), HOMO-1->LUMO (23\%) \\
4 & 246.2478632 & 0.0458 & HOMO-2->LUMO (12\%), HOMO-1->LUMO (22\%) \\
5 & 243.9992455 & 0.02 & HOMO-5->LUMO (13\%), HOMO-4->LUMO (18\%) \\
6 & 241.7960384 & 0.0035 & HOMO-7->LUMO (14\%), HOMO-5->LUMO (32\%) \\
7 & 237.3386486 & 0.0213 & HOMO-7->LUMO (43\%), HOMO-6->LUMO (11\%) \\
8 & 233.5738525 & 0.1315 & HOMO-2->LUMO+1 (14\%), HOMO->LUMO+2 (32\%) \\
9 & 226.1272988 & 0.0625 & HOMO-2->LUMO (22\%), HOMO->LUMO+2 (17\%), \\
& & & HOMO->LUMO+3 (12\%) \\
10 & 218.4112614 & 0.0547 & HOMO-1->LUMO+1 (24\%), HOMO->LUMO+8 (11\%)
\end{tabular}

Figure S46 MO energy diagram of compound 6o_OMe with the nature of the corresponding transitions 


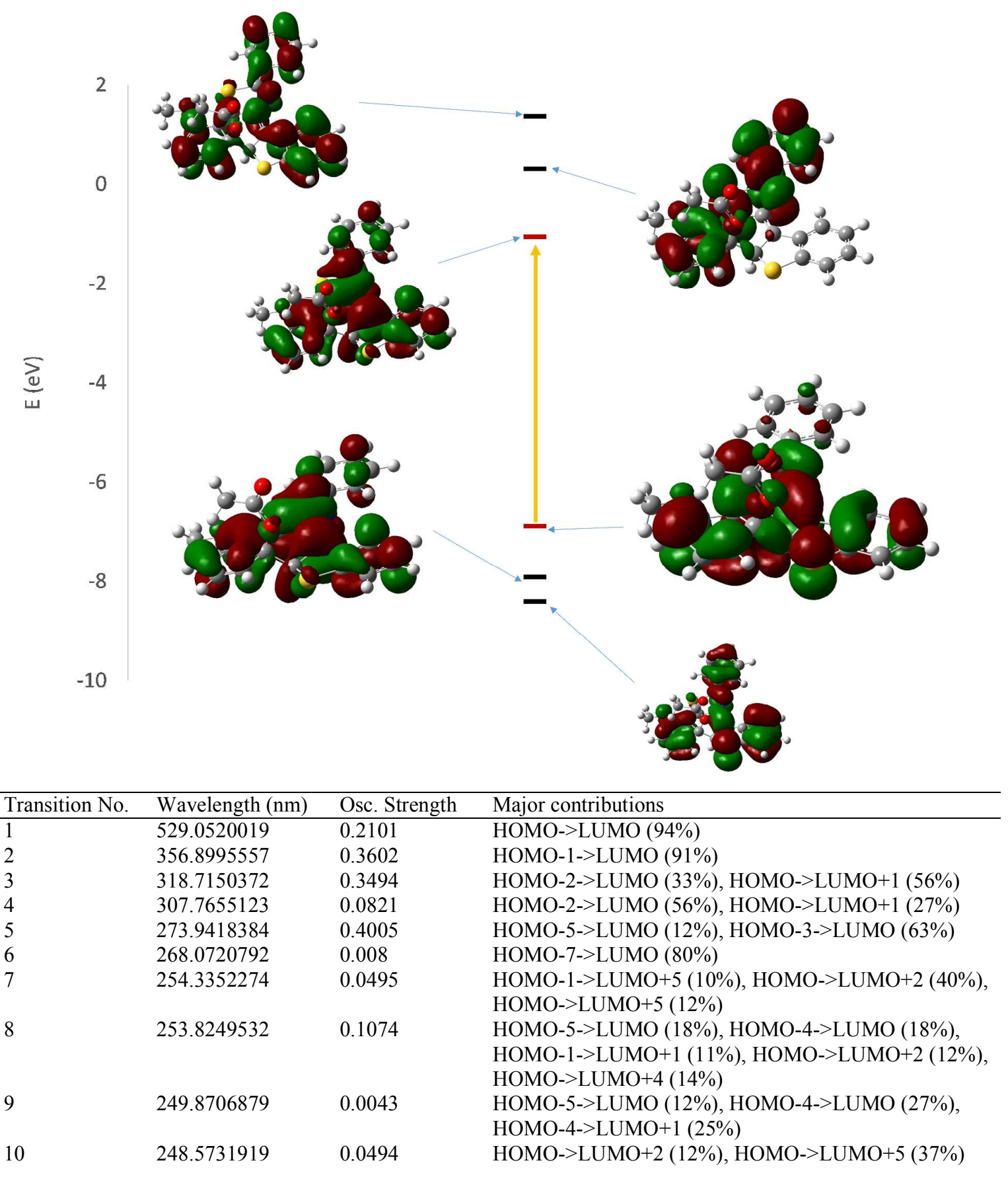

Figure $547 \mathrm{MO}$ energy diagram of compound $6 \mathrm{c}$ with the nature of the corresponding transitions 


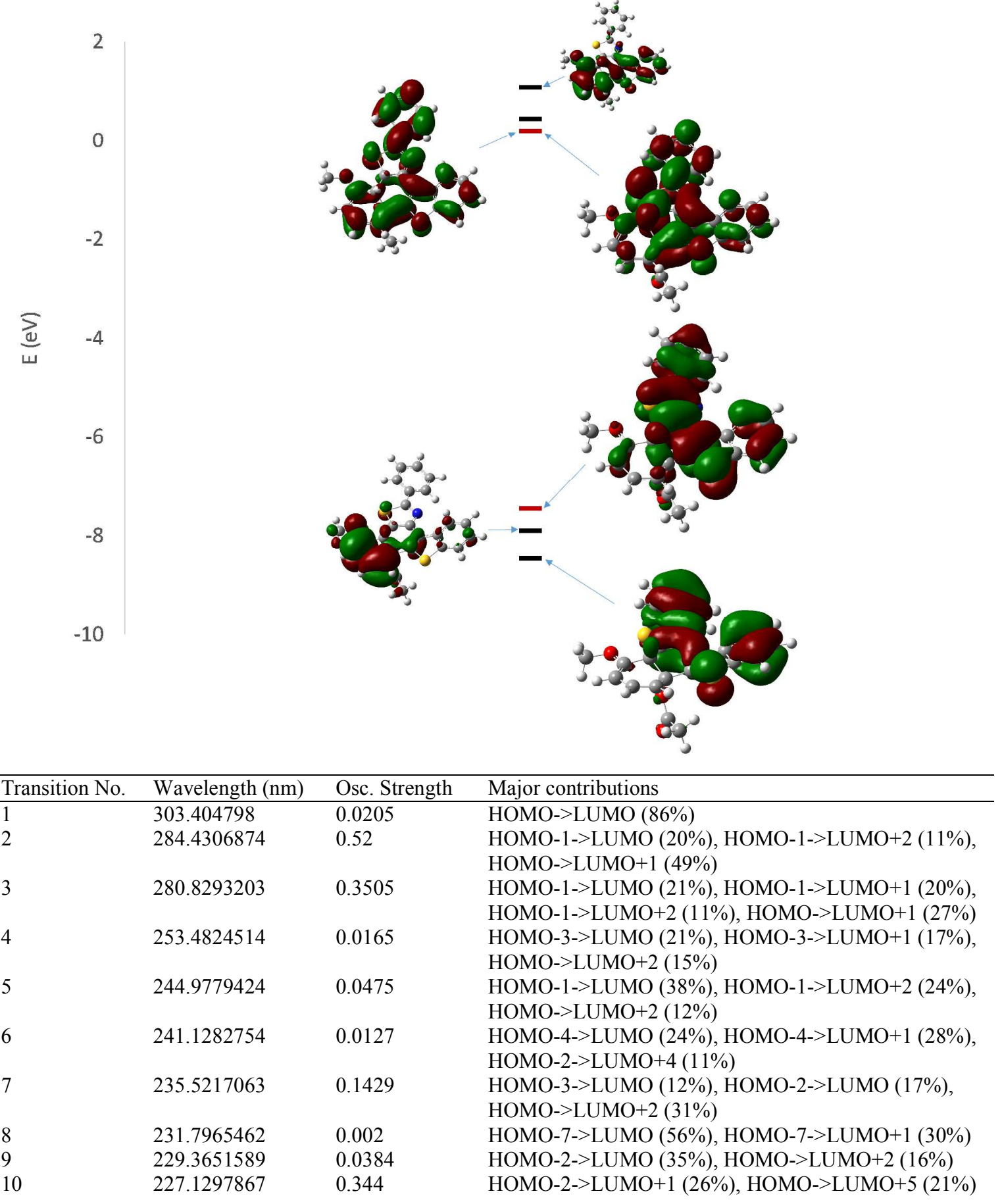

Figure $548 \mathrm{MO}$ energy diagram of compound $6 \mathrm{~s}$ with the nature of the corresponding transitions 


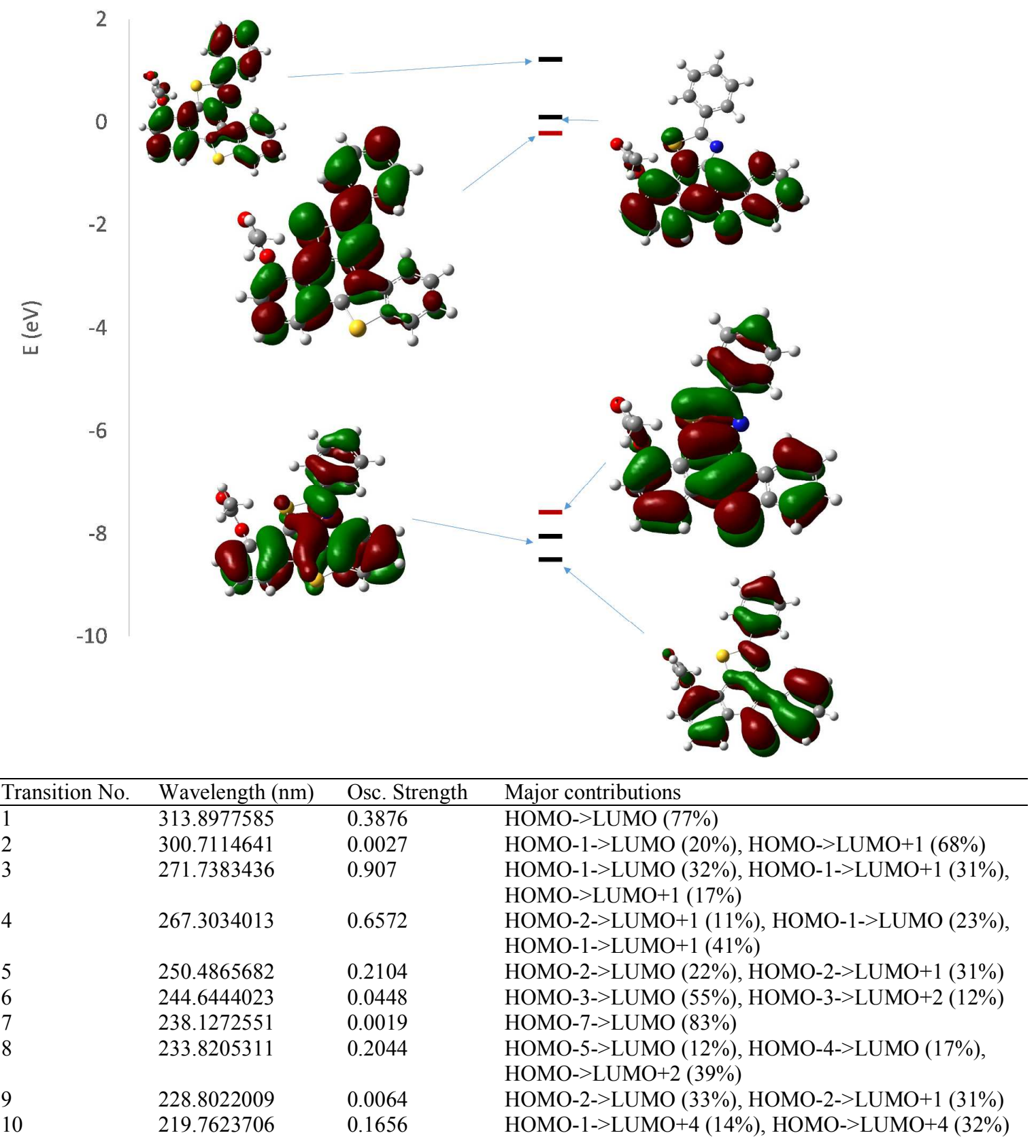

Figure $549 \mathrm{MO}$ energy diagram of compound $6 \mathrm{a}$ with the nature of the corresponding transitions 


\section{XRD data}

\section{Crystal information of coumpound 4}

\begin{tabular}{|c|c|c|c|c|}
\hline $\begin{array}{l}\text { CCDC } \\
\text { number }\end{array}$ & \multicolumn{4}{|c|}{1483014} \\
\hline $\begin{array}{c}\text { Empirical } \\
\text { Formula }\end{array}$ & \multicolumn{4}{|c|}{$\mathrm{C}_{26} \mathrm{H}_{19} \mathrm{~N} \mathrm{O}_{3} \mathrm{~S}_{2}$} \\
\hline $\begin{array}{l}\text { Formula } \\
\text { Weight }\end{array}$ & \multicolumn{4}{|c|}{429.6} \\
\hline $\begin{array}{c}\text { Crystal } \\
\text { Color, Habit }\end{array}$ & \multicolumn{4}{|c|}{ Colorless, block } \\
\hline $\begin{array}{l}\text { Crystal } \\
\text { System }\end{array}$ & \multicolumn{4}{|c|}{ triclinic } \\
\hline Lattice Type & \multicolumn{4}{|c|}{ Primitive } \\
\hline \multirow{2}{*}{$\begin{array}{c}\text { Lattice } \\
\text { Parameters }\end{array}$} & $a=10.7644(5) \AA$ & $\mathrm{b}=16.2126(7) \AA$ & $\mathrm{c}=24.4126(13) \AA$ & $\begin{array}{c}\mathrm{V}=4260.5(4) \\
\AA^{3}\end{array}$ \\
\hline & \multicolumn{3}{|c|}{$\beta=90^{\circ}$} & $\gamma=90^{\circ}$ \\
\hline Space Group & \multicolumn{4}{|c|}{$\mathrm{Pb} 21 \mathrm{a}$} \\
\hline$Z$ value & \multicolumn{4}{|c|}{8} \\
\hline$D_{\text {calc }}$ & \multicolumn{4}{|c|}{$1.339 \mathrm{~g} / \mathrm{cm}^{3}$} \\
\hline $\mathrm{F}_{000}$ & \multicolumn{4}{|c|}{1792.0} \\
\hline$\mu$ & \multicolumn{4}{|c|}{$0.272 \mathrm{~mm}^{-1}$} \\
\hline
\end{tabular}




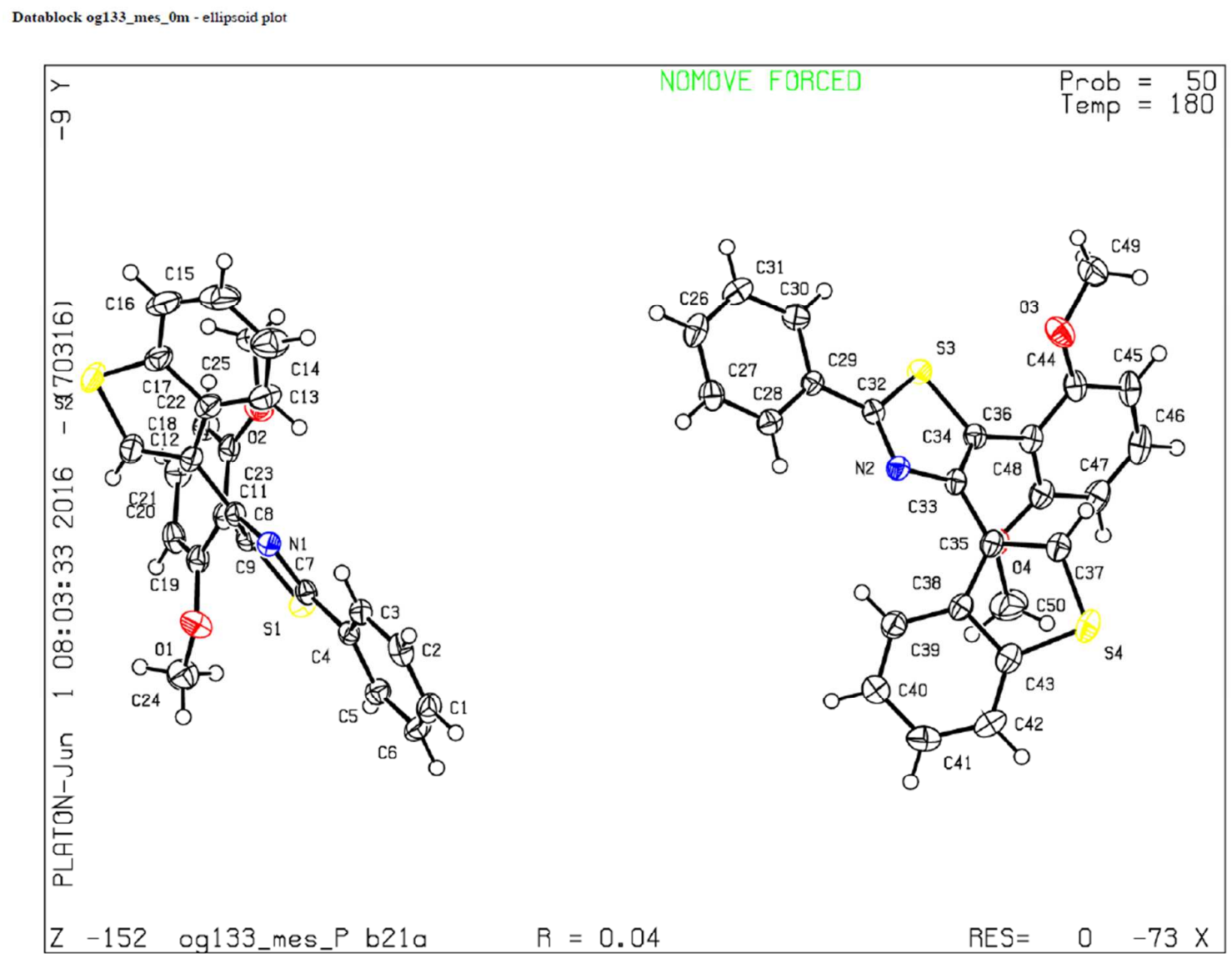

Figure S 50 Thermal ellipsoid plot of compound 4. The ellipsoids are drawn at the 50\% probability level. 


\section{Crystal information of compound 6}

\begin{tabular}{|c|c|c|c|c|}
\hline $\begin{array}{l}\text { CCDC } \\
\text { number }\end{array}$ & \multicolumn{4}{|c|}{1485482} \\
\hline $\begin{array}{l}\text { Empirical } \\
\text { Formula }\end{array}$ & \multicolumn{4}{|c|}{$\mathrm{C}_{26} \mathrm{H}_{19} \mathrm{NO}_{3} \mathrm{~S}_{2}$} \\
\hline $\begin{array}{l}\text { Formula } \\
\text { Weight }\end{array}$ & \multicolumn{4}{|c|}{457.6} \\
\hline $\begin{array}{c}\text { Crystal Color, } \\
\text { Habit }\end{array}$ & \multicolumn{4}{|c|}{ colorless, block } \\
\hline $\begin{array}{l}\text { Crystal } \\
\text { System }\end{array}$ & \multicolumn{4}{|c|}{ Triclinic } \\
\hline Lattice Type & \multicolumn{4}{|c|}{ Primitive } \\
\hline \multirow{2}{*}{$\begin{array}{c}\text { Lattice } \\
\text { Parameters }\end{array}$} & $\mathrm{a}=13.9787(5) \AA$ & $\mathrm{b}=8.8699(3) \AA$ & $\mathrm{c}=1$ & $\mathrm{~V}=2211.22(13) \AA^{3}$ \\
\hline & $\alpha=90^{\circ}$ & \multicolumn{2}{|c|}{$\beta=93.978(1)^{\circ}$} & $\gamma=90^{\circ}$ \\
\hline Space Group & \multicolumn{4}{|c|}{$\mathrm{P} 21 / \mathrm{a}$} \\
\hline $\mathrm{Z}$ value & \multicolumn{4}{|c|}{4} \\
\hline$D_{\text {calc }}$ & \multicolumn{4}{|c|}{$1.374 \mathrm{~g} / \mathrm{cm}^{3}$} \\
\hline $\mathrm{F}_{000}$ & \multicolumn{4}{|c|}{952.00} \\
\hline$\mu$ & \multicolumn{4}{|c|}{$0.270 \mathrm{~mm}^{-1}$} \\
\hline
\end{tabular}


PLATON version of $06 / 05 / 2016$; check.def file version of $05 / 05 / 2016$

Datablock I - ellipsoid plot

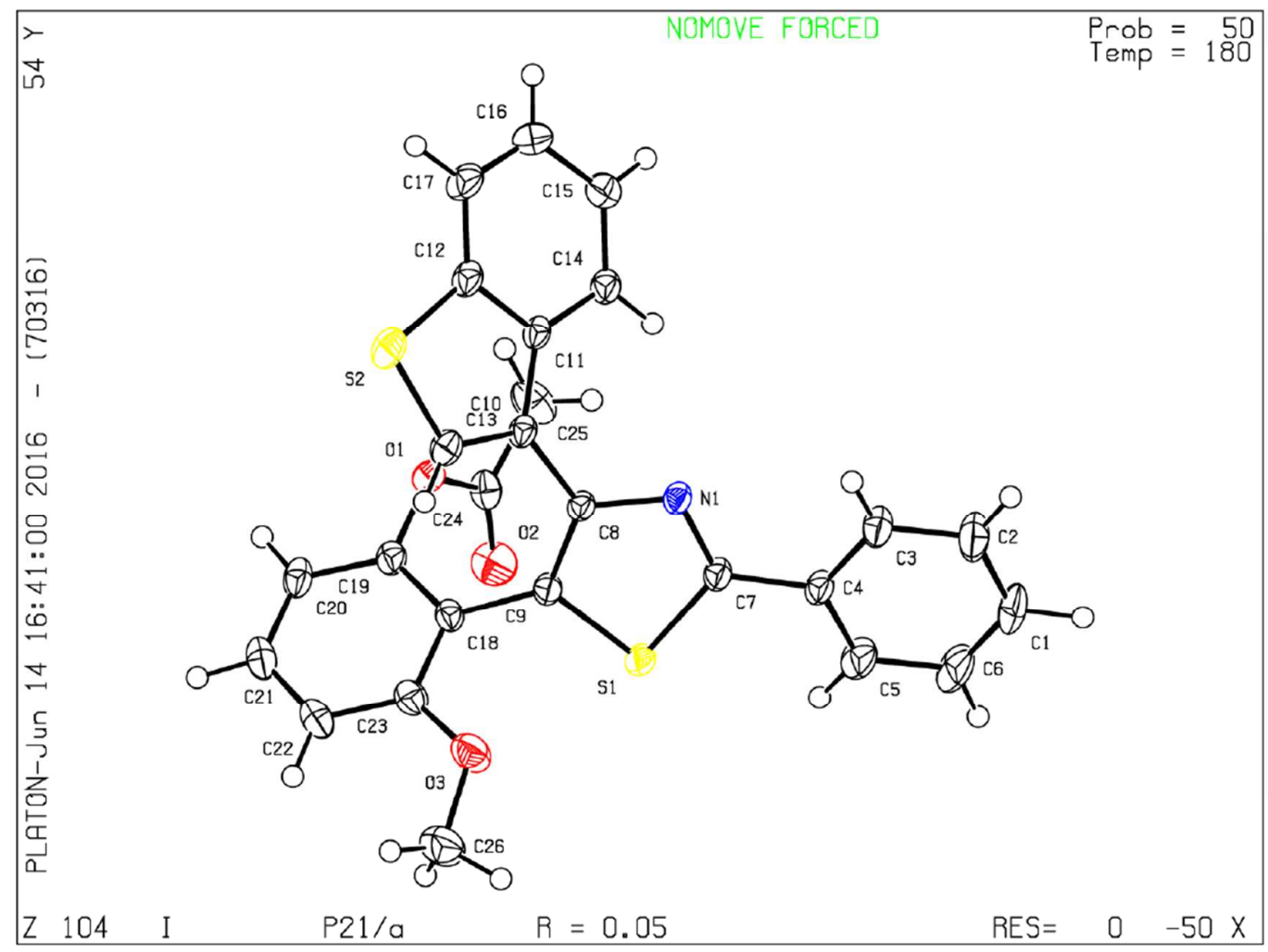

Figure S 51 Thermal ellipsoid plot of compound 6. The ellipsoids are drawn at the 50\% probability level. 